\title{
DEDUALIZING COMPLEXES AND MGM DUALITY
}

\author{
LEONID POSITSELSKI
}

\begin{abstract}
We show that various derived categories of torsion modules and contramodules over the adic completion of a commutative ring by a weakly proregular ideal are full subcategories of the related derived categories of modules. By the work of Dwyer-Greenlees and Porta-Shaul-Yekutieli, this implies an equivalence between the (bounded or unbounded) conventional derived categories of the abelian categories of torsion modules and contramodules. Over the adic completion of a commutative ring by an arbitrary finitely generated ideal, we obtain an equivalence between the derived categories of complexes of modules with torsion and contramodule cohomology modules. We also define two versions of the notion of a dedualizing complex over the adic completion of a commutative ring, one for an ideal with an Artinian quotient ring and the other one for a weakly proregular ideal, and use these to construct equivalences between the conventional as well as certain exotic derived categories of the abelian categories of torsion modules and contramodules. The philosophy of derived co-contra correspondence is discussed in the introduction.
\end{abstract}

\section{Contents}

0. Introduction

1. Derived Category of Torsion Modules 10

2. Derived Category of Contramodules 13

3. MGM Duality Theorem 22

4. Dedualizing Complexes for Ideals with Artinian Quotient Rings 26

5. Dedualizing Complexes for Weakly Proregular Ideals 34

Appendix A. Exotic Derived Categories $\quad 45$

Appendix B. Derived Functors of Finite Homological Dimension 46

References $\quad 53$

\section{INTRODUCTION}

0.0. In its simplest and purest form, the comodule-contramodule correspondence is a natural equivalence between the additive categories of injective left comodules and projective left contramodules over the same coassociative coalgebra $\mathcal{C}$ over a field $k$. The equivalence of additive categories induces an equivalence between the homotopy categories of complexes of injective comodules and projective contramodules. One would like to view this equivalence as an equivalence between the derived categories 
of left comodules and left contramodules over $\mathcal{C}$, using complexes of injective comodules and projective contramodules as resolutions. However, the former are right resolutions and the latter are left ones, so one has to work with doubly unbounded complexes. The categories of unbounded complexes of injective or projective objects are well-known to differ from the unbounded derived categories as the latter are conventionally defined. The reason is that an unbounded complex of injectives or projectives may be acyclic, yet not contractible.

Hence the relevance of the concepts of the derived categories of the second kind, or the coderived and contraderived categories, in the comodule-contramodule correspondence constructions. In well-behaved situations, the coderived category $D^{c o}(A)$ of an abelian or exact category $A$ is equivalent to the homotopy category $\operatorname{Hot}\left(A_{\text {inj }}\right)$ of complexes of injective objects in $A$, while the contraderived category $D^{\mathrm{ctr}}(B)$ of a category $B$ is equivalent to the homotopy category $\operatorname{Hot}\left(B_{\text {proj }}\right)$ of complexes of projective objects in $\mathrm{B}$. Thus the derived co-contra correspondence over a coalgebra $\mathcal{C}$ takes the form of an equivalence between the coderived category of the abelian category of left $\mathcal{C}$-comodules $\mathcal{C}$-comod and the contraderived category of the abelian category of left C-contramodules $\mathrm{C}$-contra,

$$
\mathrm{D}^{\mathrm{co}}(\mathcal{C}-\text { comod }) \simeq \mathrm{D}^{\mathrm{ctr}}(\mathcal{C}-\text { contra }) .
$$

Certain acyclic complexes of comodules and contramodules survive in the coderived and contraderived categories, and an acyclic complex is sometimes assigned to an irreducible object by the derived co-contra correspondence [21, Sections 0.2.2, 0.2.5, and 0.2.6-7] (see also [22, Sections 4.4 and 5.2]).

0.1. In more complicated relative situations mixing algebra and coalgebra features, derived co-contra correspondence theories can be often developed using the following guiding principles [25]:

(i) depending on whether one's abelian/exact category is a category of comodulelike or contramodule-like objects, one takes the coderived or the contraderived category along the coalgebra variables;

(ii) one takes the conventional unbounded derived category along the ring or algebra variables;

(iii) over a ring or coalgebra of finite homological dimension, there is no difference between the derived, coderived, and contraderived categories, so one does not have to pay attention to the distinction.

The rules (i-iii) are sufficient to build most of the derived the comodule-contramodule and semimodule-semicontramodule correspondence theorems of the book [21] (see [21, Sections 0.3.7, 5.4-5.5, and 6.3]). However, there are several simple and important situations which they do not cover.

0.2 . In the papers $[10,12]$, the homotopy categories of unbounded complexes of projective and injective modules over certain coherent or Noetherian rings were studied; and in [9], an equivalence between the homotopy categories of complexes of projective and injective modules over a ring (or two rings, in the noncommutative case) 
with a dualizing complex was obtained. In the paper [17] and the dissertation [15], these results were extended to complexes of flat and injective quasi-coherent sheaves on a Noetherian scheme; and in the paper [7, Section 2.5], to matrix factorizations. Finally, in [24, Section 5.7] this duality is formulated as a commutative diagram of equivalences between four exotic derived categories of quasi-coherent sheaves and contraherent cosheaves on a semi-separated Noetherian scheme.

The related piece of philosophy appears to look as follows. A coalgebra has been "dualized" already, being a dual thing to an algebra or a ring; so a coalgebra $\mathcal{C}$ is a dualizing complex over itself. The conventional derived category of modules over a ring is tautologically equivalent to itself, but constructing an equivalence between the coderived and contraderived categories of modules over a (say, commutative Noetherian) ring requires a dualizing complex. Having a dualizing complex over a ring makes it "more like a coalgebra". Therefore,

(iv) given a dualizing complex for a set of ring or algebra variables, one can use the coderived and the contraderived category along these variables on the two sides of the derived co-contra correspondence.

0.3. The opposite situation, when one considers the conventional unbounded derived categories of comodules and contramodules, is not as well-studied. The relation between the conventional derived categories of DG-comodules and DG-contramodules over an arbitrary DG-coalgebra over a field is discussed in (particularly, the postpublication arXiv version of) [22, Section 5.5], but it does not have the familiar form of an equivalence of triangulated categories.

In [24, Section 4.6], we construct an equivalence between the conventional (bounded or unbounded; and also absolute, etc.) derived categories of the abelian category of quasi-coherent sheaves and the exact category of contraherent cosheaves on a quasicompact semi-separated scheme $X$,

$$
\mathrm{D}^{\star}(X-\mathrm{qcoh}) \simeq \mathrm{D}^{\star}(X-\text { ctrh }) .
$$

An explanation is that, generally, a scheme "mixes the ring and coalgebra variables" in such a way that the ring(s) are responsible for the pieces being glued, while the coalgebra (or, rather, coring) governs the gluing procedure [11]. In a quasi-compact quasi-separated scheme, the gluing procedure has finite homological dimension (the quasi-coherent sheaf cohomology being a functor of finite homological dimension); so the rule (iii) "along the gluing variables" applies. Still, one additionally has to explain how to pass from the sheaves to the cosheaves and back. Over a scheme, one takes the contraherent $\mathfrak{H o m}_{X}$ from or the contratensor product $\odot_{X}$ with the structure sheaf $\mathcal{O}_{X}$ of the scheme $X[24$, Sections 2.5-6].

0.4. On the other hand, there is a stream of literature discussing torsion, completion, and duality theories for modules or sheaves over formal schemes. From the point of view elaborated in the above discussion, there seem to be two such duality theories that need to be property distinguished from each other. 
One of them is concerned with dualizing complexes on formal schemes [32, Section 5] and, being formulated in the generality of unbounded complexes of infinitely generated modules, leads to a covariant equivalence between derived categories of the second kind, extending the results of $[9,15,7]$ and $[24$, Section 5.7] to the realm of formal schemes. For affine Noetherian formal schemes, this "covariant SerreGrothendieck duality" is formulated in [24, Section C.1] (see [24, Section C.5] for a noncommutative version); and for ind-affine ind-Noetherian ind-schemes (the indspectra of pro-Noetherian topological rings), in [24, Section D.2].

To demonstrate a precise assertion here, given a dualizing complex $\mathcal{D}^{\bullet}$ for a projective system $R_{0} \longleftarrow R_{1} \longleftarrow R_{2} \longleftarrow \cdots$ of Noetherian commutative rings and surjective morphisms between them with the projective limit $\mathfrak{R}=\lim _{n} R_{n}, \quad[24$, Theorem D.2.7] claims that there is a natural equivalence between the coderived category of discrete modules and the contraderived category of contramodules over the topological ring $\mathfrak{R}$,

$$
\mathrm{D}^{\mathrm{co}}(\mathfrak{R} \text {-discr }) \simeq \mathrm{D}^{\mathrm{ctr}}(\mathfrak{R}-\text { contra }) .
$$

The other duality theory over adically complete rings and formal schemes is known as the Matlis-Greenlees-May duality [14, 6, 18]. The aim of the present paper is to formulate it explicitly as an equivalence between the conventional derived categories of the abelian categories of torsion modules and contramodules over the adic completions of certain commutative rings. Thus the MGM duality is viewed as a species of the "naïve derived co-contra correspondence" of [24, Section 4.6].

0.5. Let us devote a few paragraphs to a more substantive discussion of the issues involved. Let $X$ be a scheme and $Z \subset X$ be a closed subscheme. Denote by $U=X \backslash Z$ the open complement to $Z$ in $X$. Then the formal completion of $X$ along $Z$ can be viewed heuristically as the complement to $U$ in $X$,

$$
X_{Z}=X \backslash U \text {. }
$$

In particular, the quasi-coherent torsion sheaves on $X_{Z}$ are, almost by the definition, those quasi-coherent sheaves on $X$ whose restrictions vanish on $U$.

Passing to the triangulated categories and denoting by $k: U \longrightarrow X$ the open embedding morphism, we notice that under weak assumptions on $k$ the inverse image functor $k^{*}: \mathrm{D}(X-\mathrm{qcoh}) \longrightarrow \mathrm{D}(U$-qcoh $)$ between the derived categories of quasicoherent sheaves on $X$ and $U$ has a right adjoint functor of derived direct image $\mathbb{R} k_{*}: \mathrm{D}(U$-qcoh $) \longrightarrow \mathrm{D}(X-\mathrm{qcoh})$. The composition $k^{*} \circ \mathbb{R} k_{*}$ is isomorphic to the identity functor on $\mathrm{D}(U-\mathrm{qcoh})$, so $k^{*}$ is a Verdier quotient functor and the functor $\mathbb{R} k_{*}$ is fully faithful. To be more precise, one would probably want to have the scheme $U$ quasi-compact and the scheme $X$ semi-separated, or otherwise the scheme $X$ locally Noetherian, for the functor $\mathbb{R} k_{*}$ to be well-behaved; and under slightly stronger assumptions one can prove existence of a Neeman extraordinary inverse image functor $k^{!}: \mathrm{D}(X-\mathrm{qcoh}) \longrightarrow \mathrm{D}(U-\mathrm{qcoh})$ right adjoint to $\mathbb{R} k_{*}[16]$.

It follows immediately that the kernels of the functors $k^{*}$ and $k^{!}$are two equivalent subcategories in $\mathrm{D}(X-\mathrm{qcoh})$ (a purely algebraic description of this picture can 
be found in [18, Section 8]). Furthermore, the functor of inverse image of contraherent cosheaves $k^{!}: \mathrm{D}(X$-ctrh $) \longrightarrow \mathrm{D}(U$-ctrh $)$ has a left adjoint functor of derived direct image $\mathbb{L} k_{!}: \mathrm{D}(U$-ctrh $) \longrightarrow \mathrm{D}(X-$ ctrh $)$. The equivalences of derived categories $\mathrm{D}(X-\mathrm{qcoh}) \simeq \mathrm{D}(X-$ ctrh $)$ and $\mathrm{D}(U-\mathrm{qcoh}) \simeq \mathrm{D}(U$-ctrh $)$ from Section 0.3 transform the functor $\mathbb{R} k_{*}$ into the functor $\mathbb{L} k_{!}$, identifying the two functors $k^{!}$and providing a rather explicit construction of Neeman's inverse image [24, Section 4.8].

This is not yet a promised equivalence between the derived categories of a pair of abelian or exact categories, however. E. g., one still has to prove that the kernel of the functor $k^{*}$ is equivalent to the derived category $\mathrm{D}\left(X-\mathrm{qcoh}_{Z \text {-tors }}\right)$ of quasi-coherent tor-

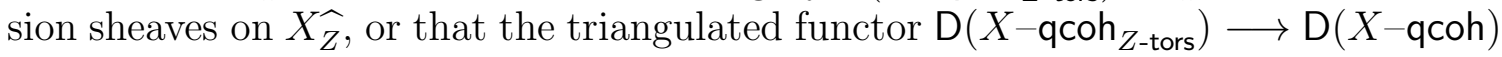
induced by the embedding of abelian categories $X$-qcoh $Z$-tors $\longrightarrow X$-qcoh is fully faithful. For bounded derived categories $\mathrm{D}^{\mathrm{b}}$ or $\mathrm{D}^{+}$in place of $\mathrm{D}$ and a Noetherian scheme $X$ this is not difficult, as one can use the Artin-Rees lemma to show that injectives in $X$-qcoh $Z$-tors are also injective in $X$-qcoh; for the unbounded derived categories, the question appears to be more involved. One also has to identify the kernel of the functor $k$ ! with the derived category of the abelian or exact category of contramodules or contraherent cosheaves of contramodules on $X_{Z}^{\widehat{~}}$.

0.6. Let us now continue the discussion from a different angle. When the scheme $X$ is quasi-compact and quasi-separated, and its open subscheme $U \subset X$ is quasicompact, the above heuristics suggest that the formal scheme $X_{Z}^{\widehat{y}}$, being the result of "subtracting" $U$ from $X$, has the homological dimension of its "coalgebra variables" bounded, approximately, by the sum of the cardinality parameters of the open coverings of $X$ and $U$, and consequently finite. To use another visual metaphor, one can say that the formal completion of $X$ along $Z$ is obtained by cutting from $X$ a small tubular neighborhood around $Z$. The observation is that the scissors used to perform the cut tend to have finite homological dimension.

Once again, the Artin-Rees lemma, claiming that the completion functor is exact, would seem to provide an even stronger assertion for finitely generated modules, but we are interested in infinitely generated ones (even over a Noetherian ring). The precise conditions needed to prove such a result are a more technical issue. In the case of an affine scheme $X=\operatorname{Spec} R$ our heuristics seem to suggest that the open subscheme $U=X \backslash Z$ should be quasi-compact, i. e., the ideal $I \subset R$ defining the closed subscheme $Z \subset X$ should be finitely generated. In assumptions, admittedly, stronger than this, still weaker than the Noetherianity of $R$, a pair of such homological finite-dimensionality assertions on the comodule and contramodule sides is indeed proven in [18, Corollaries 4.28 and 5.27].

0.7. No homological dimension condition, though, can automatically solve the problem of constructing an equivalence between the derived categories of two different abelian categories, even if these are the categories of comodules and contramodules over the same coalgebra-like algebraic structure. The piece of data that is missing here is what we call a dedualizing complex. 
The coderived category of left comodules and the contraderived category of left contramodules over a coalgebra over a field are always equivalent to each other; but constructing an equivalence between the conventional derived categories of comodules and contramodules over a (co-Noetherian or cocoherent) coalgebra requires a dedualizing complex. A ring is a dedualizing complex over itself; having a dedualizing complex for a coalgebra makes it more like a ring. In other words,

(v) given a dedualizing complex for a set of coalgebra variables, one can have a "naive" derived co-contra correspondence with the conventional derived categories of comodules and contramodules along these variables on the two sides of a triangulated equivalence.

The definition of a dedualizing complex $\mathcal{B}^{\bullet}$ is, approximately, dual to that of a dualizing one. It has to be a finite complex of comodules or bicomodules. There being, generally speaking, no projective objects in comodule categories, one cannot ask $\mathcal{B} \bullet$ to be a finite complex of projective objects, but it has to have finite projective dimension as a complex in the bounded derived category. It has to satisfy a unitality condition imposed on the graded ring of its endomorphisms in the derived category. Finally, there should be a finiteness condition similar or dual to the coherence condition on the cohomology sheaves of a dualizing complex on a Noetherian scheme. The latter one seems to be the hardest to formulate, and the approaches may vary.

Let us emphasize that the dualizing and the dedualizing complexes play rather different roles in the respective theories. The purpose of the dualizing complex is to mitigate the infinite homological dimension problem, bridging the gap between the coderived and the contraderived category. The dedualizing complex is there to bridge the gap between the abelian categories of comodules and contramodules. In both cases, the problem to be solved by the choice of a specific complex appears due to a mismatch between the kind of abelian categories being considered and the kind of derived category constructions being applied. Between the coderived category of comodules and the contraderived category of contramodules (say, over a coalgebra over a field), there is no gap to be bridged.

0.8. We have yet to explain how a dedualizing complex on a formal scheme is to be obtained. For dualizing complexes on algebraic varieties $X$ over a field $k$, the classical prescription is to consider the structure morphism $p: X \longrightarrow$ Spec $k$ and set $\mathcal{D}_{X}^{\bullet}=p^{+} \mathcal{O}_{\text {Spec } k}$, where $p^{+}$denotes the Deligne extraordinary inverse image functor

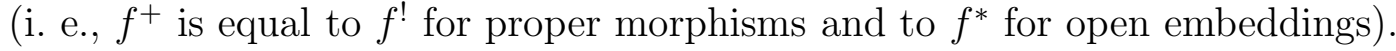

A similar rule works for formal completions of algebraic varieties: given a scheme $X$ with a dualizing complex $\mathcal{D}_{X}^{\bullet}$ and a closed subscheme $Z \subset X$, a dualizing complex on the formal scheme $X_{Z}^{\widehat{C}}$ can be constructed as the derived subcomplex with settheoretic supports $\mathcal{D}_{X_{Z}}^{\bullet}=\mathbb{R} i^{!} \mathcal{D}_{X}^{\bullet}$, where $i$ denotes the embedding $X_{Z} \longrightarrow X$. As to the dedualizing complex on $X_{Z}^{\widehat{Z}}$, it is produced by applying the functor $\mathbb{R} i$ to the structure sheaf of the scheme $X$,

$$
\mathcal{B}_{X_{Z}}^{\bullet}=\mathbb{R} i^{!} \mathcal{O}_{X}, \quad i: X_{Z} \longrightarrow X .
$$


Note that the formal completion $X_{Z} \subset X$ of a closed subscheme $Z$ in a scheme $X$ can be considered both as a kind of "closed subscheme" or "open subscheme" in $X$, depending on a point of view. The above construction of the dedualizing complex can be explained by saying that one would "prefer" to use the $*$-restriction of quasi-

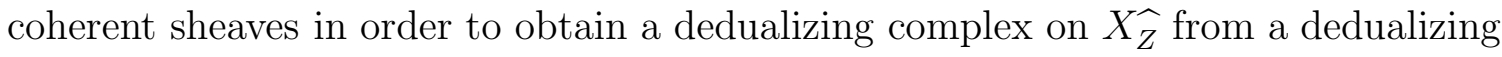
complex on $X$; but given that a dedualizing complex on a formal scheme must be a complex of quasi-coherent torsion sheaves, one is "forced" to use the !-restriction, which is a "satisfactory substitution" inasmuch as the natural morphism $X_{Z}^{\widehat{Z}} \longrightarrow X$ can be viewed as a species of open embedding.

The complex $\mathcal{B}_{X_{Z}}^{\bullet}$ for the formal completion of an affine scheme $X=$ Spec $R$ along its closed subscheme $Z \subset X$ defined by a finitely generated ideal $I \subset R$ plays a crucial role in the MGM duality papers $[6,18]$. Traditionally, it is viewed as an object of the derived category of $R$-modules represented by an explicit Koszul/telescope complex of infinitely generated free $R$-modules $\operatorname{Tel}^{\bullet}(R, \mathbf{s})$ concentrated in the cohomological degrees from 0 to $m$, where $\mathbf{s}=\left\{s_{1}, \ldots, s_{m}\right\}$ is a chosen set of generators of the ideal $I$, and having $I$-torsion cohomology modules. With the perspective of extending the MGM duality to nonaffine schemes in mind, certainly it is preferable to work with torsion modules and contramodules over the $I$-adic completion of $R$ only, avoiding the use of any other $R$-modules such as the infinitely generated free ones. Dependence on a fixed set of generators $\mathbf{s}$ of the ideal $I$, which may not exist globally along a closed subscheme $Z$ in a nonaffine scheme $X$, is also undesirable.

0.9. Let us now describe the contents of this paper and our main results in some detail. For a commutative ring $R$ with a weakly proregular finitely generated ideal $I \subset R$, Porta, Shaul, and Yekutieli construct an equivalence between (what they call) the full subcategories of cohomologically I-torsion complexes $\mathrm{D}(R \text {-mod })_{I \text {-tors }} \subset \mathrm{D}(R$-mod $)$ and cohomologically I-adically complete complexes $\mathrm{D}(R \text {-mod })_{I \text {-com }} \subset \mathrm{D}(R-\mathrm{mod})$ in the unbounded derived category of $R$-modules $\mathrm{D}(R-\bmod )$,

$$
\mathrm{D}(R-\bmod )_{I \text {-tors }} \simeq \mathrm{D}(R-\text { mod })_{I \text {-com }}
$$

[18, Theorems 1.1 and 7.11]. Moreover, they consider the full abelian subcategory of $I$-torsion $R$-modules $R$-mod $I$-tors $\subset R$-mod in the abelian category of $R$-modules, and show that the full subcategory of cohomologically $I$-torsion complexes coincides with the full subcategory of complexes with $I$-torsion cohomology modules in $\mathrm{D}(R$-mod),

$$
\mathrm{D}(R-\mathrm{mod})_{I \text {-tors }}=\mathrm{D}_{I \text {-tors }}(R-\text { mod }) \subset \mathrm{D}(R-\text { mod })
$$

[18, Corollary 4.32]; a similar result was earlier obtained in [6, Proposition 6.12].

Our first result in Section 1 is that, for a weakly proregular finitely generated ideal $I$ in a commutative ring $R$, the derived category of the abelian category of $I$-torsion $R$-modules is a full subcategory in $\mathrm{D}(R$-mod $)$ coinciding with the full subcategory of complexes with $I$-torsion cohomology modules,

$$
\mathrm{D}_{I \text {-tors }}(R-\bmod )=\mathrm{D}\left(R-\bmod _{I \text {-tors }}\right) \subset \mathrm{D}(R-\bmod ) .
$$


Furthermore, in Section 2 we define the full abelian subcategory of $I$-contramodules (or $I$-contramodule $R$-modules) $R$ - $\bmod _{I \text {-ctra }} \subset R$-mod and show that the derived category of $I$-contramodule $R$-modules is a full subcategory of the derived category of $R$-modules,

$$
\mathrm{D}\left(R-\bmod _{I-\mathrm{ctra}}\right) \subset \mathrm{D}(R-\bmod ) .
$$

Moreover, the full subcategory of cohomologically $I$-adically complete complexes coincides with the full subcategory of complexes with $I$-contramodule cohomology modules in $\mathrm{D}(R-$ mod) and with (the image of) the derived category of the abelian category of $I$-contramodule $R$-modules,

$$
\mathrm{D}(R-\bmod )_{I-\text { com }}=\mathrm{D}_{I-\text { ctra }}(R-\bmod )=\mathrm{D}\left(R-\bmod _{I-\text { ctra }}\right) .
$$

(concerning the first equality, cf. [6, Proposition 6.15]).

Combining these results, we obtain a natural equivalence between the (bounded or unbounded) conventional derived categories of the abelian categories of $I$-torsion and $I$-contramodule $R$-modules,

$$
\mathrm{D}^{\star}\left(R-\bmod _{I \text {-tors }}\right) \simeq \mathrm{D}^{\star}\left(R-\bmod _{I \text {-ctra }}\right) .
$$

Let us point out that the equivalence of triangulated subcategories (1) in the derived category $\mathrm{D}(R$-mod) can be actually established for any finitely generated ideal $I$ in a commutative ring $R$, as it follows from the above discussion in Section 0.5. This was shown already by Dwyer and Greenlees in [6, Theorem 2.1, Section 4.1, and Section 6], and we explain this anew in our Section 3. In fact, we prove that for any finitely generated ideal $I \subset R$ the full subcategories of complexes with torsion and contramodule cohomology modules are naturally equivalent,

$$
\mathrm{D}_{I \text {-tors }}^{\star}(R-\text { mod }) \simeq \mathrm{D}_{I \text {-ctra }}^{\star}(R \text {-mod }) .
$$

However, the weak proregularity condition appears to be essential for constructing an equivalence between the derived categories of the abelian categories $R-\bmod _{I \text {-tors }}$ and $R-\bmod _{I \text {-ctra }}$ in the formula (2).

0.10. Notice that the formal completions of algebraic varieties over a field $k$ at their closed points defined over $k$ are precisely the ind-spectra of finitely cogenerated conilpotent cocommutative coalgebras over $k$. (See [5, Section I.6] for a discussion of what we would now call the equivalence between the categories of ind-finite indschemes over a field and cocommutative coalgebras over this field.) Thus coalgebras occur in connection with closed points on algebraic varieties, while the adic completions with respect to ideals correspond to arbitrary closed subsets.

As the above discussion in this introduction suggests, the notion of a dedualizing complex in the case of (not even necessarily cocommutative, but coassociative) coalgebras over a field is easier to approach than for adic completions of commutative rings. In fact, thinking about the coalgebra case was an important stepping stone for the development of the author's ideas about the subject.

Still, from the point of view of many a reader the necessity to delve into an additional, largely unrelated background and recall the basics of coalgebras on the way to the desired definition from commutative algebra may feel like an unnecessary burden. 
Therefore, we decided to move our treatment of the MGM duality for coalgebras to a separate paper [28], while restricting ourselves here to a brief mention of this theory and its main result in this section of the introduction.

The definition of a dedualizing complex as a complex of bicomodules over two coalgebras satisfying a list of conditions is presented in [28]. Given a dedualizing complex $\mathcal{B} \bullet$ for a pair of cocoherent coalgebras $\mathcal{C}$ and $\mathcal{D}$, an equivalence between the bounded or unbounded, conventional or absolute derived categories of left comodules over $\mathcal{C}$ and left contramodules over $\mathcal{D}$ is obtained,

$$
\mathrm{D}^{\star}(\mathrm{C}-\text { comod }) \simeq \mathrm{D}^{\star}(\mathcal{D}-\text { contra }) \text {. }
$$

Instead of the coalgebra story, we have included in this paper an intermediate Section 4 , where a theory very similar to the MGM duality for coalgebras is developed in the case of a Noetherian ring $R$ with an ideal $I$ such that the quotient ring $R / I$ is Artinian (cf. [23, Section 1.1]). This assumption, even though very restrictive, allows to give a conceptually clear definition of a dedualizing complex, which can serve as a prototype for the more clumsy condition applicable in the general case.

In the final Section 5, we work out the dedualizing complexes-based approach to MGM duality theory over the adic completions of commutative rings by arbitrary weakly proregular finitely generated ideals. Even though our definition of a dedualizing complex in this setting is not as neat as in the cases of two coalgebras or an ideal with the Artinian quotient ring, it allows to obtain a second proof of the equivalence of derived categories (2). Moreover, while the proof based on the arguments of Section 3 applies to the conventional derived categories only, the approach in Section 5 produces an equivalence of the absolute derived categories as well.

A brief summary of the definitions of exotic derived categories used in the main body of the paper is included in Appendix A. A formalism of derived functors needed for the constructions of the last two sections is developed in Appendix B.

For a general discussion of the abelian categories of contramodules, we refer to the survey paper [26]. In the role of an introduction to the coderived, contraderived and absolute derived categories the reader can use [21, Sections 0.2 and 2.1] or, better yet, [22, Sections 3-5]; for more advanced results, one can look into [7, Sections 1.3-1.6] or [24, Appendix A]. An overview exposition on the derived comodule-contramodule correspondence phenomenon can be found in the presentation [25].

Acknowlegdement. The author is grateful to all the people who brought the MGM duality to his attention over the several recent years, including Sergey Arkhipov, Joseph Bernstein, and Amnon Yekutieli. This work was started when I was visiting Ben Gurion University of the Negev in Be'er Sheva in the Summer of 2014 and continued when I was supported by a fellowship from the Lady Davis Foundation at the Technion in October 2014-March 2015. I also wish to thank the anonymous referee for several helpful suggestions. 


\section{Derived Category of Torsion Modules}

Let $R$ be a commutative ring and $I \subset R$ be an ideal. An $R$-module $M$ is said to be $I$-torsion if for every pair of elements $s \in I$ and $x \in M$ there exists an integer $n \geqslant 1$ such that $s^{n} x=0$ in $M$. The full subcategory of $I$-torsion submodules in $R$-mod is denoted by $R-\bmod _{I \text {-tors }}$. Clearly, the subcategory $R-\bmod _{I \text {-tors }}$ is closed under the operations of passage to submodules, quotient modules, extensions, and infinite direct sums in $R$-mod. Therefore, $R$ - $\bmod _{I \text {-tors }}$ is an abelian category with exact functors of infinite direct sum and its embedding $R-\bmod _{I \text {-tors }} \longrightarrow R$-mod is an exact functor preserving the infinite direct sums.

Following [18], we denote by $\Gamma_{I}(M)$ the maximal $I$-torsion submodule in an arbitrary $R$-module $M$. The functor $\Gamma_{I}: R-\bmod \longrightarrow R-\bmod _{I \text {-tors }}$ is left exact; it is the right adjoint functor to the embedding functor $R$ - $\bmod _{I \text {-tors }} \longrightarrow R$-mod.

Assume that the ideal $I$ is finitely generated. Then an $R$-module $M$ is $I$-torsion if and only if for every element $x \in M$ there exists $n \geqslant 1$ such that $I^{n} x=0$ in $M$.

Let $s_{1}, \ldots, s_{m} \in R$ be a finite sequence of elements in $R$ generating the ideal $I$. For simplicity of notation, we will denote the sequence $s_{1}, \ldots, s_{m}$ by a single letter $\mathbf{s}$. For any $R$-module $M$, consider the following Cech complex $C_{\mathbf{s}}^{\bullet}(M)$

$$
\bigoplus_{j=1}^{m} M\left[s_{j}^{-1}\right] \longrightarrow \bigoplus_{j^{\prime}<j^{\prime \prime}} M\left[s_{j^{\prime}}^{-1}, s_{j^{\prime \prime}}^{-1}\right] \longrightarrow \cdots \longrightarrow M\left[s_{1}^{-1}, \ldots, s_{m}^{-1}\right] .
$$

We place the first term $\bigoplus_{j} M\left[s_{j}^{-1}\right]$ of the complex $C_{\mathbf{s}}(M)$ in the cohomological degree 0 , so that there is a natural morphism of complexes (coaugmentation or unit) $k: M \longrightarrow C_{\mathbf{s}}^{\bullet}(M)$, and denote by $C_{\mathbf{s}}(M)^{\sim}$ the cocone of this morphism,

$$
M \longrightarrow \bigoplus_{j=1}^{m} M\left[s_{j}^{-1}\right] \longrightarrow \bigoplus_{j^{\prime}<j^{\prime \prime}} M\left[s_{j^{\prime}}^{-1}, s_{j^{\prime \prime}}^{-1}\right] \longrightarrow \cdots \longrightarrow M\left[s_{1}^{-1}, \ldots, s_{m}^{-1}\right] \text {. }
$$

So the complex $C_{\mathbf{s}}^{\bullet}(M)^{\sim}$ also has its first term $M$ at the cohomological degree 0 . Obviously, there are natural isomorphisms of complexes $C_{\mathbf{s}}(M) \simeq C_{\mathbf{s}}(R) \otimes_{R} M$ and $C_{\mathbf{s}}(M)^{\sim} \simeq C_{\mathbf{s}}(R)^{\sim} \otimes_{R} M$. Furthermore, the complex $C_{\mathbf{s}}^{\bullet}(R)^{\sim}$ is isomorphic to the tensor product of the similar complexes corresponding to one-element sequences $\left\{s_{j}\right\}$ over all the elements of the sequence $s_{1}, \ldots, s_{m}$

$$
C_{\mathbf{s}}^{\bullet}(R)^{\sim} \simeq C_{\left\{s_{1}\right\}}^{\bullet}(R)^{\sim} \otimes_{R} \cdots \otimes_{R} C_{\left\{s_{m}\right\}}^{\bullet}(R)^{\sim} .
$$

Lemma 1.1. (a) For any $R$-module $M$, all the cohomology modules $H^{*} C_{\mathbf{s}}(M)^{\sim}$ of the complex $C_{\mathbf{s}}^{\bullet}(M)^{\sim}$ are $I$-torsion $R$-modules.

(b) For any $R$-module $M$, the natural morphism of complexes $C_{\mathbf{s}}(M)^{\sim} \longrightarrow M$ induces an isomorphism of $R$-modules

$$
H^{0} C_{\mathbf{s}}^{\bullet}(M)^{\sim} \simeq \Gamma_{I}(M) .
$$

(c) For any I-torsion $R$-module $M$, the morphism of complexes $C_{\mathbf{s}}^{\bullet}(M)^{\sim} \longrightarrow M$ is an isomorphism.

Proof. To prove part (a), notice that the complex $C_{\mathbf{s}}^{\bullet}(M)^{\sim}\left[s_{j}^{-1}\right]$ is contractible for every $j$, because $C_{\left\{s_{j}\right\}}(R)^{\sim}\left[s_{j}^{-1}\right]$ is a contractible two-term complex $\left(R\left[s_{j}^{-1}\right] \rightarrow R\left[s_{j}^{-1}\right]\right)$. To obtain part (b), recall that for any given element $s \in R$ the kernel of the map $M \longrightarrow M\left[s^{-1}\right]$ consists precisely of all the elements $x \in M$ for which there exists an 
integer $n \geqslant 1$ such that $s^{n} x=0$. Part (c) is obvious, as the complex $C_{\mathbf{s}}^{\bullet}(M)$ vanishes entirely for any $I$-torsion $R$-module $M$.

Denote by $X$ the affine scheme Spec $R$, by $Z=$ Spec $R / I \subset X$ the corresponding closed subscheme in $X$, by $U=X \backslash Z$ its open complement, and by $U_{j}$ the principal affine open subschemes $\operatorname{Spec} R\left[s_{j}^{-1}\right] \subset \operatorname{Spec} R$. The open subschemes $U_{j} \subset X$ form an affine open covering of the open subscheme $U \subset X$. Let $\mathcal{M}$ denote the quasicoherent sheaf on $X$ corresponding to the $R$-module $M$; then the Cech complex $C_{\mathbf{s}}^{\bullet}(M)$ computes the sheaf cohomology $H^{*}\left(U,\left.\mathcal{M}\right|_{U}\right)$.

In particular, when $R$ is a Noetherian ring and $J$ is an injective $R$-module, the related quasi-coherent sheaf $\mathcal{J}$ on $X$ is flasque and $\left.\mathcal{J}\right|_{U}$ is an injective quasi-coherent sheaf on $U$ [8, Lemma II.7.16 and Theorem II.7.18]. Hence it follows that the complex $C_{\mathbf{s}}^{\bullet}(J)$ is quasi-isomorphic to its zero cohomology module $H^{0} C_{\mathbf{s}}^{\bullet}(J) \simeq \mathcal{J}(U)$ and the complex $C_{\mathbf{s}}^{\bullet}(J)^{\sim}$ is quasi-isomorphic to the two-term complex $\mathcal{J}(X) \longrightarrow \mathcal{J}(U)$, which also has its only cohomology group in degree 0 . We have shown that $H^{n} C_{\mathbf{s}}^{\bullet}(J)^{\sim}=0$ for $n>0$. A direct algebraic proof of this assertion (based on the same Matlis' classification of injective modules over Noetherian rings [13] as Hartshorne's argument in [8]) can be found in [18, Theorem 4.34].

According to one of the equivalent definitions, a finite sequence of elements $\mathbf{s}$ in a commutative ring $R$ is said to be weakly proregular if the cohomology groups of the complex $C_{\mathbf{s}}^{\bullet}(J)^{\sim}$ vanish in the positive cohomological degrees for all injective $R$-modules $J$ (see [30, Theorem 3.2(ii)] or [18, Theorem 4.24]). According to [18, Corollary 6.2] (see also [30, Corollary 3.3]), the weak proregularity property of a finite sequence $\mathbf{s}$ in a commutative ring $R$ only depends on the ideal $I$ that this sequence generates, and in fact, even only on the ideal $\sqrt{I} \subset R$. We have seen that any ideal $I$ in a Noetherian ring $R$ is weakly proregular. Another equivalent definition of weak proregularity will appear in Section 2.

Lemma 1.2. (a) For any weakly proregular finite sequence of elements $\mathbf{s}$ in a commutative ring $R$, the complex $C_{\mathbf{s}}^{\bullet}(M)^{\sim}$ assigned to an $R$-module $M$ computes the right derived functor $\mathbb{R}^{*} \Gamma_{I}(M)$ of the left exact functor $\Gamma_{I}$, viewed as taking values in the category $R$-mod.

(b) For any weakly proregular finitely generated ideal $I$ in a commutative ring $R$, (the right derived functor $\mathbb{R}^{*} \Gamma_{I}$ of) the left exact functor $\Gamma_{I}: R$-mod $\longrightarrow R$ - $\bmod _{I \text {-tors }}$ has finite homological dimension not exceeding the minimal number of generators of the ideal $I$.

Proof. This is [30, Theorem 3.2(iii)] or [18, Corollaries 4.26 and 4.28]. Part (a): given an $R$-module $M$, consider its right injective resolution $J^{\bullet}$. Then the total complex of the bicomplex $C_{\mathbf{s}}^{\bullet}\left(J^{\bullet}\right)^{\sim}$ is quasi-isomorphic both to the complex $\Gamma_{I}\left(J^{\bullet}\right)$, since the morphism $\Gamma_{I}\left(J^{n}\right) \longrightarrow C_{\mathbf{s}}^{\bullet}\left(J^{n}\right)^{\sim}$ is a quasi-isomorphism for every $n$, and to the complex $C_{\mathbf{s}}^{\bullet}(M)^{\sim}$, because the functor $N \longmapsto C_{\mathbf{s}}^{\bullet}(N)^{\sim}$ takes exact sequences of $R$-modules $N$ to exact sequences of complexes $C_{\mathbf{s}}^{\bullet}(-)^{\sim}$. As the embedding functor $R-\bmod _{I \text {-tors }} \longrightarrow R$-mod is exact and takes nonzero objects to nonzero objects, part (b) immediately follows. 
For the most part of Sections 1-2, we will consider simultaneously and almost on equal footing a number of conventional and exotic derived category constructions introduced in $[21,22]$, and [24, Appendix A]. The definitions of these derived categories are briefly recalled in Appendix A to this paper. A reader unfamiliar with derived categories of the second kind will not loose much restricting his attention to the conventional derived categories $\mathrm{D}^{\mathrm{b}}, \mathrm{D}^{+}, \mathrm{D}^{-}$, and $\mathrm{D}$, and forgetting about the rest. In Section 3, we will only work with the four conventional derived categories; and then return to a list of symbols including both the conventional and the absolute derived categories in the final Sections 4-5 and Appendix B.

Theorem 1.3. Let $R$ be a commutative ring and $I \subset R$ be a weakly proregular finitely generated ideal. Then for any symbol $\star=\mathrm{b},+,-, \varnothing$, abs + , abs - , co, or abs, the triangulated functor $\mathrm{D}^{\star}\left(R-\bmod _{I \text {-tors }}\right) \longrightarrow \mathrm{D}^{\star}(R$-mod $)$ induced by the embedding of abelian categories $R-\bmod _{I \text {-tors }} \longrightarrow R$-mod is fully faithful.

Proof. In the case of the derived categories $\mathrm{D}^{\mathrm{b}}$ or $\mathrm{D}^{+}$over a Noetherian ring $R$, it suffices to notice that, according to the Artin-Rees lemma, the functor $R-\bmod _{I \text {-tors }} \longrightarrow$ $R$-mod preserves injectivity of objects (cf. [26, Proposition 2.2.2(a)]). For the other derived categories in our list, Lemma 1.2(b) essentially says that the question "has finite homological dimension" and therefore "reduces to finite complexes". A formal proof in the general case proceeds as follows.

Denote by $R$ - $\bmod _{I \text {-tors-adj }}$ the full subcategory of objects adjusted to $\Gamma_{I}$ in $R$-mod; in other words, the subcategory $R$ - $\bmod _{I \text {-tors-adj }} \subset R$-mod consists of all the $R$-modules $M$ for which $\mathbb{R}^{n} \Gamma_{I}(M)=0$ for $n>0$, or equivalently, $H^{n} C_{\mathbf{s}}^{\bullet}(M)^{\sim}=0$ for $n>0$. The full subcategory $R-\bmod _{I \text {-tors-adj }}$ is closed under extensions, cokernels of injective morphisms, and infinite direct sums in $R$-mod. Hence the subcategory $R$-mod $\operatorname{m-tors-adj}_{\text {- }}$ inherits an exact category structure of the abelian category $R$-mod.

Moreover, by Lemma 1.2(b) any $R$-module has a finite right resolution of uniformly bounded length by objects of the subcategory $R-\bmod _{I \text {-tors-adj }}$ It follows that for any symbol $\star$ on our list the triangulated functor $\mathrm{D}^{\star}\left(R-\bmod _{I \text {-tors-adj }}\right) \longrightarrow \mathrm{D}^{\star}(R-\bmod )$ induced by the exact embedding $R-\bmod _{I \text {-tors-adj }} \longrightarrow R$-mod is an equivalence of triangulated categories [24, Proposition A.5.6],

$$
\mathrm{D}^{\star}\left(R-\bmod _{I \text {-tors-adj }}\right) \simeq \mathrm{D}^{\star}(R-\bmod ) .
$$

Obviously, the restriction of functor $\Gamma_{I}$ to the full exact subcategory $R-\bmod _{I \text {-tors-adj }}$ $\subset R$-mod is an exact functor $\Gamma_{I}: R$ - $\bmod _{I \text {-tors-adj }} \longrightarrow R$ - $\bmod _{I \text {-tors }}$. Applying the functor $\Gamma_{I}$ to complexes of modules from the category $R$ - $\bmod _{I \text {-tors-adj }}$ termwise, we obtain the right derived functor

$$
\mathbb{R} \Gamma_{I}: \mathrm{D}^{\star}(R-\bmod ) \longrightarrow \mathrm{D}^{\star}\left(R-\bmod _{I \text {-tors }}\right) .
$$

As the passage to derived functors in the sense of Deligne [4, $\left.\mathrm{n}^{\mathrm{os}} 1.2 .1-2\right]$ generally preserves adjoint pairs of functors, the derived functor $\mathbb{R} \Gamma_{I}$ is right adjoint to the triangulated functor $\mathrm{D}^{\star}\left(R\right.$ - $\left.\bmod _{I \text {-tors }}\right) \longrightarrow \mathrm{D}^{\star}(R-$ mod $)$ induced by the embedding of abelian categories $R-\bmod _{I \text {-tors }} \longrightarrow R-\bmod$ (see also [21, Lemma 8.3]). 
Furthermore, by Lemma $1.1(\mathrm{c})$ the abelian subcategory $R$ - $\bmod _{I \text {-tors }} \subset R$-mod is contained in the exact subcategory $R-\bmod _{I \text {-tors-adj }}$,

$$
R-\bmod _{I \text {-tors }} \subset R-\bmod _{I \text {-tors-adj }} \text {. }
$$

Therefore, the composition of adjoint functors

$$
\mathrm{D}^{\star}\left(R-\bmod _{I \text {-tors }}\right) \longrightarrow \mathrm{D}^{\star}(R-\bmod ) \longrightarrow \mathrm{D}^{\star}\left(R-\bmod _{I \text {-tors }}\right)
$$

is isomorphic to the identity functor on $\mathrm{D}^{\star}\left(R-\bmod _{I \text {-tors }}\right)$. It follows immediately that the functor $\mathrm{D}^{\star}\left(R-\bmod _{I \text {-tors }}\right) \longrightarrow \mathrm{D}^{\star}(R-\bmod )$ is fully faithful, while the functor $\mathbb{R} \Gamma_{I}: \mathrm{D}^{\star}(R-\bmod ) \longrightarrow \mathrm{D}^{\star}\left(R-\bmod _{I \text {-tors }}\right)$ is a Verdier quotient functor.

Corollary 1.4. Let $R$ be a commutative ring and $I \subset R$ be a weakly proregular finitely generated ideal. Then for any symbol $\star=\mathrm{b},+,-$, or $\varnothing$, the triangulated functor $\mathrm{D}^{\star}\left(R-\bmod _{I \text {-tors }}\right) \longrightarrow \mathrm{D}^{*}(R-\bmod )$ identifies the derived category $\mathrm{D}^{\star}\left(R-\bmod _{I \text {-tors }}\right)$ with the full subcategory $\mathrm{D}_{I \text {-tors }}^{\star}(R$-mod $) \subset \mathrm{D}^{\star}(R$-mod $)$ consisting of all the complexes with the cohomology modules belonging to $R-\bmod _{I \text {-tors }}$.

Proof. This is [18, Corollary 4.32]; see also [6, Proposition 6.12]. It is obvious that the image of the functor $\mathrm{D}^{\star}\left(R-\bmod _{I \text {-tors }}\right) \longrightarrow \mathrm{D}^{\star}(R-\bmod )$ is contained in $\mathrm{D}_{I \text {-tors }}^{\star}(R$-mod $)$. To prove the converse inclusion, one notices that, according to the proof of Theorem 1.3 , the image of the fully faithful functor $\mathrm{D}^{\star}\left(R-\bmod _{I \text {-tors }}\right) \longrightarrow \mathrm{D}^{\star}(R-\bmod )$ consists precisely of all the complexes fixed by the composition of adjoint functors $\mathrm{D}^{\star}(R-\bmod ) \longrightarrow \mathrm{D}^{\star}\left(R-\bmod _{I \text {-tors }}\right) \longrightarrow \mathrm{D}^{\star}(R-$ mod $)$. Since the functor $\mathbb{R} \Gamma_{I}$ has finite homological dimension, a complex viewed as an object of the conventional derived category is fixed by this composition whenever its cohomology modules are.

\section{Derived Category of Contramodules}

An abelian group $P$ with an additive operator $s: P \longrightarrow P$ is said to be an $s$-contramodule if for every sequence of elements $p_{0}, p_{1}, p_{2}, \ldots \in P$ there is a unique sequence of elements $q_{0}, q_{1}, q_{2}, \ldots \in P$ satisfying the infinite system of nonhomogeneous linear equations

$$
q_{n}=s q_{n+1}+p_{n} \quad \text { for all } n \geqslant 0 .
$$

The infinite summation operation with s-power coefficients in an s-contramodule $P$ is defined by the rule

$$
\sum_{n=0}^{\infty} s^{n} p_{n}=q_{0} \in P
$$

Conversely, given an additive, associative, and unital infinite summation operation

$$
\left(p_{n}\right)_{n=0}^{\infty} \longmapsto \sum_{n=0}^{\infty} s^{n} p_{n}
$$

in an abelian group $P$ one can uniquely solve the system of equations (4) by setting

$$
q_{n}=\sum_{i=0}^{\infty} s^{i} p_{n+i}
$$

(see [26, Section 1.6] and [23, Section B.5]; cf. [24, Section C.2]). 
A module $P$ over a ring $R$ with a chosen element $s \in R$ is said to be an $s$-contramodule if it is a contramodule with respect to the operator of multiplication with $s$. When $s$ is a central element in $R$, this condition is equivalent to the Ext group vanishing $\operatorname{Ext}_{R}^{i}\left(R\left[s^{-1}\right], P\right)=0$ for $i=0$ and 1 (notice that the $R$-module $R\left[s^{-1}\right]$ has projective dimension at most 1 , so such Ext groups with $i \geqslant 2$ always vanish) [23, Lemma B.7.1].

Let $R$ be a commutative ring, $I$ be an ideal in $R$, and $s_{j} \in R$ be a set of generators of the ideal $I$. Then the property of an $R$-module $P$ to be a contramodule with respect to all the elements $s_{j}$ depends only on the ideal $I \subset R$ and not on the choice of a particular set of its generators. Indeed, it suffices to check this assertion for rings finitely generated over the ring of integers $\mathbb{Z}$, and for Noetherian rings it follows from [23, Theorem B.1.1] (see also [26, Section 2.2]). Another proof of this assertion will be obtained as a byproduct of the arguments below in this section.

So an $R$-module $P$ is called an $I$-contramodule, or an $I$-contramodule $R$-module, if it is a contramodule with respect to some set of generators of the ideal $I$. Making use of the definition of contramodules in terms of Ext vanishing, one easily deduces the assertion that the property of an $R$-module to be an $I$-contramodule only depends on the radical $\sqrt{I} \subset R$ of the ideal $I$.

The full subcategory of $I$-contramodule $R$-modules $R$-mod $I$-ctra $\subset R$-mod is closed under the kernels and cokernels of morphisms, extensions, and infinite products in $R$-mod. Hence $R$ - $\bmod _{I \text {-ctra }}$ is an abelian category with exact functors of infinite product and its embedding $R-\bmod _{I \text {-ctra }} \longrightarrow R$-mod into the category of $R$-modules is an exact functor preserving the infinite products.

Let $M$ and $P$ be two $R$-modules. Then whenever either $M$ is $I$-torsion, or $P$ is an $I$-contramodule, the $R$-module $\operatorname{Hom}_{R}(M, P)$ is an $I$-contramodule. Indeed, for any element $s \in I$ the infinite summation operations with $s$-power coefficients in $\operatorname{Hom}_{R}(M, P)$ are provided by the formula

$$
\left(\sum_{n=0}^{\infty} s^{n} f_{n}\right)(x)=\sum_{n=0}^{\infty} f_{n}\left(s^{n} x\right),
$$

where the sum in the right-hand side only has a finite number of nonvanishing summands, in the former case, and

$$
\left(\sum_{n=0}^{\infty} s^{n} f_{n}\right)(x)=\sum_{n=0}^{\infty} s^{n} f_{n}(x),
$$

where the sum in the right-hand side is the $s$-power infinite summation operation in the $s$-contramodule $P$, in the latter one. Here $f_{n}$ are arbitrary elements of the $R$-module $\operatorname{Hom}_{R}(M, P)$ and $x$ is an arbitrary element of $M$.

Assume that the ideal $I \subset R$ is finitely generated. In any $R$-module that is separated and complete in the $I$-adic topology, i. e., an $R$-module $P$ for which the natural map $P \longrightarrow \lim _{n} P / I^{n} P$ is an isomorphism, the infinite summation operation with $s$-power coefficients can be defined for any $s \in I$ as the $I$-adic limit of finite partial sums. So all the $I$-adically separated and complete $R$-modules are $I$-contramodules. Conversely, all the $I$-contramodule $R$-modules are $I$-adically complete, but they do not have to be $I$-adically separated (see [18, Example 4.33] or [26, Section 1.5] and 
the references therein). Still, one has $P / I P \neq 0$ for any $I$-contramodule $R$-module $P \neq 0$ (see [20, Corollary 2.5], [23, Lemma 1.3.1], or [26, Lemma 2.1(b)]).

The theory of "cohomologically $I$-adically complete complexes of $R$-modules" is developed in [18] using the $I$-adic completion functor $\Lambda_{I}: M \longmapsto \lim _{n} M / I^{n} M$, which is neither left, nor right exact (and generally seems to be only well-behaved for flat $R$-modules $M)$. We prefer to construct and use the functor left adjoint to the embedding functor of the full subcategory of $I$-contramodule $R$-modules.

Proposition 2.1. Assume that the ideal $I \subset R$ is finitely generated. Then the embedding functor $R-\bmod _{I-\mathrm{ctra}} \longrightarrow R$-mod has a left adjoint functor

$$
\Delta_{I}: R-\bmod \longrightarrow R-\bmod _{I-\text { ctra }} \text {. }
$$

Proof. We will present an explicit construction of the functor $\Delta_{I}$. Let us first consider the case of an ideal $I$ generated by a single element $s \in R$. To any $R$-module $M$ we assign the $R$-module morphism

$$
\phi_{M}^{s}: \prod_{n=1}^{\infty} M \longrightarrow \prod_{n=0}^{\infty} M
$$

taking every sequence of elements $r_{1}, r_{2}, \ldots \in M$ to the sequence of elements

$$
m_{0}=-s r_{1}, \quad m_{1}=r_{1}-s r_{2}, \quad m_{2}=r_{2}-s r_{3}, \ldots
$$

Denote by $\Delta_{s}(M)$ the cokernel of the morphism $\phi_{M}^{s}$.

Let us show, first of all, that the $R$-module $\Delta_{s}(M)$ is an $s$-contramodule. The assertion only depends on the $\mathbb{Z}[s]$-module structure on $M$, so one can assume that $R=\mathbb{Z}[s]$. For any abelian group $A$, consider the $\mathbb{Z}[s]$-module $A[s]$ of polynomials in $s$ with the coefficients in $A$. It is straightforward to compute that $\Delta_{s}(A[s])=A[[s]]$ is the $\mathbb{Z}[s]$-module of formal Taylor power series in $s$ with the coefficients in $A$; the isomorphism is provided by the map $\prod_{n=0}^{\infty} A[s] \longrightarrow A[[s]]$ taking any sequence of polynomials $m_{0}, m_{1}, m_{2}, \ldots$ to the power series $\sum_{n=0}^{\infty} s^{n} m_{n}(s)$. Clearly, any $\mathbb{Z}[s]$-module is the cokernel of a morphism of modules of the form $A[s]$, the functor $\Delta_{s}$ preserves cokernels, and the $\mathbb{Z}[s]$-modules $A[[s]]$ are $s$-contramodules. Since the class of $s$-contramodules is closed under cokernels, the assertion is proven.

Now let us show that the group of $R$-module morphisms $\operatorname{Hom}_{R}(M, P)$ is naturally isomorphic to $\operatorname{Hom}_{R}\left(\Delta_{s}(M), P\right)$ whenever an $R$-module $P$ is an $s$-contramodule. The embedding $M \longrightarrow \prod_{n=0}^{\infty} M$ taking every element $m \in M$ to the sequence $m_{0}=m$, $m_{1}=m_{2}=\cdots=0$, induces a natural morphism $M \longrightarrow \Delta_{s}(M)$ for any $R$-module $M$. For any $s$-contramodule $P$, any $R$-linear map $f: M \longrightarrow P$ is extended to an $R$-linear map $g: \Delta_{s}(M) \longrightarrow P$ by the rule

$$
g\left(m_{0}, m_{1}, m_{2}, \ldots\right)=\sum_{n=0}^{\infty} s^{n} f\left(m_{n}\right),
$$

where the summation sign in the right-hand side stands for the infinite summation operation in an $s$-contramodule $P$.

It remains to show that there exists no other $R$-linear map $\Delta_{s}(M) \longrightarrow P$ whose composition with the natural map $M \longrightarrow \Delta_{s}(M)$ is equal to the given $R$-linear 
map $f: M \longrightarrow P$. Indeed, let $h: \Delta_{s}(M) \longrightarrow P$ be such a map; we will denote its composition with the natural surjection $\prod_{n=0}^{\infty} M \longrightarrow \Delta_{s}(M)$ also by $h$. Set

$$
q_{n}=h\left(m_{n}, m_{n+1}, m_{n+2}, \ldots\right) \in P \quad \text { for every } n \geqslant 0 .
$$

The map $\phi_{M}^{s}$ takes the sequence of elements $\left(r_{1}, r_{2}, \ldots\right)=\left(m_{n+1}, m_{n+2}, \ldots\right)$ to the sequence

$$
\phi\left(m_{n+1}, m_{n+2}, \ldots\right)=\left(0, m_{n+1}, m_{n+2}, \ldots\right)-s\left(m_{n+1}, m_{n+2}, \ldots\right),
$$

so the elements $q_{n}$ and $f\left(m_{n}\right) \in P$ satisfy the system of linear equations

$$
q_{n}-f\left(m_{n}\right)=s q_{n+1} \quad \text { for all } n \geqslant 0 .
$$

By the definition of an $s$-contramodule, the sequence of elements $q_{n}$ is uniquely determined by the sequence $f\left(m_{n}\right)$.

We have constructed the functor $\Delta_{I}=\Delta_{s}$ for an ideal $I=(s)$ generated by a single element $s \in R$. To produce the functor $\Delta_{I}$ for an ideal $I=\left(s_{j}\right)_{j=1}^{m}$ generated by a finite sequence of elements $s_{j} \in R$, notice that for any two elements $s$ and $t \in R$ the functor $\Delta_{s}: R-\bmod \longrightarrow R-\bmod _{(s) \text {-ctra }} \subset R$-mod takes the full subcategory $R-\bmod _{(t) \text {-ctra }} \subset R$-mod into itself. Indeed, the full subcategory $R$ - $\bmod _{(t) \text {-ctra }}$ is closed under the infinite products and cokernels in $R$-mod.

Hence it is clear that the restriction of the functor $\Delta_{s}$ to the full subcategory $R-\bmod _{(t) \text {-ctra }} \subset R$-mod provides a functor $R-\bmod _{(t) \text {-ctra }} \longrightarrow R$ - $\bmod _{(s, t) \text {-ctra }}$ left adjoint to the embedding of full subcategory $R-\bmod _{(s, t) \text {-ctra }} \longrightarrow R-\bmod _{(t) \text {-ctra }}$. Composing the functors $\Delta_{s_{j}}$ over all the elements in a chosen finite set of generators of the ideal $I \subset R$, we obtain the desired functor $\Delta_{I}=\Delta_{s_{1}} \cdots \Delta_{s_{m}}$.

In the paper [18, Definition 5.1], the notation $\mathrm{Tel}^{\bullet}(R, s)$ is used for the following two-term complex of infinitely generated free $R$-modules sitting in the cohomological degrees 0 and 1

$$
\bigoplus_{n=0}^{\infty} R \delta_{n} \longrightarrow \bigoplus_{n=0}^{\infty} R \delta_{n}
$$

with the differential $d\left(\delta_{0}\right)=\delta_{0}, d\left(\delta_{n}\right)=\delta_{n-1}-s \delta_{n}$ for $n \geqslant 1$. Essentially the same complex can be found in [6, equation (6.7)]. Passing to the quotient modules of both the terms of this complex by the submodules $R \delta_{0}$ and redenoting $\epsilon_{n}=\delta_{n+1}$ in the left-hand side, we obtain a homotopy equivalent complex $T^{\bullet}(R, s)$ of the form

$$
\bigoplus_{n=0}^{\infty} R \epsilon_{n} \longrightarrow \bigoplus_{n=1}^{\infty} R \delta_{n}
$$

with the differential $d\left(\epsilon_{0}\right)=-s \delta_{1}, d\left(\epsilon_{n}\right)=\delta_{n}-s \delta_{n+1}$ for $n \geqslant 1$.

The telescope complex $\operatorname{Tel}^{\bullet}(R, s)$ is interesting because it is quasi-isomorphic to the two-term Čech complex $C_{\{s\}}^{\bullet}(R)^{\sim}=\left(R \rightarrow R\left[s^{-1}\right]\right)$ from Section 1; the quasiisomorphism $\mathrm{Tel}^{\bullet}(R, s) \longrightarrow C_{\{s\}}^{\bullet}(R)^{\sim}$ is given by the rules $\delta_{0} \longmapsto 1, \delta_{n} \longmapsto 0$ in degree 0 and $\delta_{n} \longmapsto s^{-n}$ in degree 1. Setting $\operatorname{Tel}^{\bullet}(R, \mathbf{s})=\operatorname{Tel}^{\bullet}\left(R, s_{1}\right) \otimes_{R} \cdots \otimes_{R}$ $\operatorname{Tel}^{\bullet}\left(R, s_{m}\right)$ and $T^{\bullet}(R, \mathbf{s})=T^{\bullet}\left(R, s_{1}\right) \otimes_{R} \cdots \otimes_{R} T^{\bullet}\left(R, s_{m}\right)$ for a finite sequence $s_{1}, \ldots$, $s_{m} \in R$, we have a quasi-isomorphism of finite complexes of flat $R$-modules

$$
\operatorname{Tel}^{\bullet}(R, \mathbf{s}) \longrightarrow C_{\mathbf{s}}^{\bullet}(R)^{\sim}
$$


and a homotopy equivalence of finite complexes of free $R$-modules

$$
\operatorname{Tel}^{\bullet}(R, \mathbf{s}) \longrightarrow T^{\bullet}(R, \mathbf{s}) \text {. }
$$

Lemma 2.2. (a) For any $R$-module $M$, all the homology modules of the complex $\operatorname{Hom}_{R}\left(\operatorname{Tel}^{\bullet}(R, \mathbf{s}), M\right)$ are $I$-contramodule $R$-modules.

(b) For any $R$-module $M$, there is a natural isomorphism of $R$-modules

$$
H_{0} \operatorname{Hom}_{R}\left(\mathrm{Tel}^{\bullet}(R, \mathbf{s}), M\right) \simeq \Delta_{I}(M) .
$$

(c) For any $I$-contramodule $R$-module $P$, the morphism of complexes $\operatorname{Tel}^{\bullet}(R, \mathbf{s}) \longrightarrow$ $R$ induces a quasi-isomorphism of complexes of $R$-modules

$$
P \longrightarrow \operatorname{Hom}_{R}\left(\mathrm{Tel}^{\bullet}(R, \mathbf{s}), P\right) \text {. }
$$

Proof. Part (a): for any element $s \in R$, denote by $T^{\bullet}(R, s)^{\prime}$ the subcomplex of the two-term complex $\operatorname{Tel}^{\bullet}(R, s)$ spanned by all the generators $\delta_{n}$ with the exception of the generator $\delta_{0}$ in degree 0 . The complex $T^{\bullet}(R, s)^{\prime}$ a free $R$-module resolution of the $R$-module $R\left[s^{-1}\right]$. Consequently, an $R$-module $P$ is an $s$-contramodule if and only if the two-term complex $\operatorname{Hom}_{R}\left(T^{\bullet}(R, s)^{\prime}, P\right)$ is acyclic, that is the $R$-module morphism $\operatorname{Hom}_{R}\left(T^{1}(R, s)^{\prime}, P\right) \longrightarrow \operatorname{Hom}_{R}\left(T^{0}(R, s)^{\prime}, P\right)$ is an isomorphism. It follows that a complex of $R$-modules $P^{\bullet}$ has $s$-contramodule cohomology modules if and only if the morphism of complexes $\operatorname{Hom}_{R}\left(T^{1}(R, s)^{\prime}, P^{\bullet}\right) \longrightarrow \operatorname{Hom}_{R}\left(T^{0}(R, s)^{\prime}, P^{\bullet}\right)$ is a quasi-isomorphism, that is the complex $\operatorname{Hom}_{R}\left(T^{\bullet}(R, s)^{\prime}, P^{\bullet}\right)$ is acyclic. Now for any $R$-module $M$ and any $1 \leqslant j \leqslant m$ one has $\operatorname{Hom}_{R}\left(T^{\bullet}\left(R, s_{j}\right)^{\prime}, \operatorname{Hom}_{R}\left(\operatorname{Tel}^{\bullet}(R, \mathbf{s}), M\right)\right) \simeq$ $\operatorname{Hom}_{R}\left(\operatorname{Tel}^{\bullet}(R, \mathbf{s}) \otimes_{R} T^{\bullet}\left(R, s_{j}\right)^{\prime}, M\right)$, and the complex Tel ${ }^{\bullet}(R, \mathbf{s}) \otimes_{R} T^{\bullet}\left(R, s_{j}\right)^{\prime}$, being a complex of free $R$-modules quasi-isomorphic to the contractible complex $C_{\mathbf{s}}^{\bullet}(R)^{\sim}\left[s_{j}^{-1}\right]$ (see the proof of Lemma 1.1(a)), is also contractible.

Part (b): the functor $\Delta_{s}$ from the proof of Proposition 2.1 is isomorphic to the functor $M \longmapsto H_{0} \operatorname{Hom}_{R}\left(T^{\bullet}(R, s), M\right)$ by construction, as the map $\phi_{M}^{s}$ is precisely the differential in the two-term complex $\operatorname{Hom}_{R}\left(T^{\bullet}(R, s), M\right)$. Consequently, the functor $\Delta_{I}$ is isomorphic to the functor $M \longmapsto H_{0} \operatorname{Hom}_{R}\left(T^{\bullet}(R, \mathbf{s}), M\right)$ when the ideal $I$ is generated by a sequence of elements $s_{1}, \ldots, s_{m} \in R$. The latter functor is isomorphic to the functor $M \longmapsto H_{0} \operatorname{Hom}_{R}\left(\operatorname{Tel}^{\bullet}(R, \mathbf{s}), M\right)$, because the complexes $\operatorname{Tel}^{\bullet}(R, \mathbf{s})$ and $T^{\bullet}(R, \mathbf{s})$ are homotopy equivalent.

Part (c): all the terms of the complexes on the both sides of the quasi-isomorphism $\mathrm{Tel}^{\bullet}(R, \mathbf{s}) \longrightarrow C_{\mathbf{s}}^{\bullet}(R)^{\sim}$ have the property that all the modules $\operatorname{Ext}_{R}^{n}$ from them into any $I$-contramodule $R$-module $P$ in the positive degrees $n>0$ vanish, so the complexes $\operatorname{Hom}_{R}\left(\mathrm{Tel}^{\bullet}(R, \mathbf{s}), P\right)$ and $\operatorname{Hom}_{R}\left(C_{\mathbf{s}}^{\bullet}(R)^{\sim}, P\right)$ are quasi-isomorphic. Furthermore, all the terms of the complex $C_{\mathbf{s}}^{\bullet}(R)$ in the kernel of the natural morphism of complexes $C_{\mathbf{s}}^{\bullet}(R)^{\sim} \longrightarrow R$ have the property that all the modules Ext $_{R}^{n}$ from them into any $I$-contramodule $R$-module $P$ in all the degrees $n \geqslant 0$ vanish, so the complex $\operatorname{Hom}_{R}\left(C_{\mathbf{s}}(R)^{\sim}, P\right)$ is quasi-isomorphic to $P$.

In particular, we have obtained another proof of the fact that the property of an $R$-module to be a contramodule with respect to each element $s_{j}$ in a finite sequence $s_{1}, \ldots, s_{m} \in R$ only depends on the radical $\sqrt{I} \subset R$ of the ideal $I$ generated by the 
elements $s_{1}, \ldots, s_{m}$ in $R$. Indeed, according to Lemma 2.2(b) together with the proof of Proposition 2.1, an $R$-module $P$ is a contramodule with respect to $s_{1}, \ldots, s_{m}$ if and only if it has the form $H_{0} \operatorname{Hom}_{R}\left(\mathrm{Tel}^{\bullet}(R, \mathbf{s}), M\right)$ for some $R$-module $M$. Since the complexes $\mathrm{Tel}^{\bullet}(R, \mathbf{s})$ for various sequences $\mathbf{s}$ corresponding to the same ideal $\sqrt{I}$ are homotopy equivalent [18, Theorem 6.1], it follows that the contramodule property only depends on the ideal $\sqrt{I}$. (Alternatively, one can use Lemma $2.2(\mathrm{a})$ and (c).)

A projective system of $R$-modules $M_{1} \longleftarrow M_{2} \longleftarrow M_{3} \longleftarrow \cdots$ indexed by the positive integers is said to be pro-zero if for every $l \geqslant 1$ there exists $n>l$ such that the composition of maps $M_{l} \longleftarrow \cdots \longleftarrow M_{n}$ vanishes. Both the countable projective limit functor and its (first and only) derived functor vanish on pro-zero projective systems, $\lim _{n} M_{n}=0=\lim _{n}^{1} M_{n}$.

The two-term complex $\operatorname{Tel}^{\bullet}(R, s)$ is the union of its subcomplexes of free $R$-modules $\operatorname{Tel}_{n}^{*}(R, s)$ generated by the symbols $\delta_{0}, \ldots, \delta_{n}$ in the degrees 0 and 1 . Accordingly, the complex $\operatorname{Tel}^{\bullet}(R, \mathbf{s})$ is the union of its subcomplexes of finitely generated free $R$-modules $\operatorname{Tel}_{n}^{\bullet}\left(R, s_{1}\right) \otimes_{R} \cdots \otimes_{R} \operatorname{Tel}_{n}^{\bullet}\left(R, s_{m}\right)$. The dual complexes of finitely generated free $R$-modules $\operatorname{Hom}_{R}\left(\operatorname{Tel}_{n}^{\bullet}(R, \mathbf{s}), R\right)$, concentrated in the homological degrees from 0 to $m$, form a projective system. A finite sequence of elements $\mathbf{s}$ in a commutative ring $R$ is called weakly proregular if the projective system of homology modules $H_{i} \operatorname{Hom}_{R}\left(\operatorname{Tel}_{n}^{\mathbf{*}}(R, \mathbf{s}), R\right.$ ) is pro-zero for every $i>0$ (see [30, Definition 2.3] or [18, Definition 4.21 and Lemma 5.7]. The following lemma shows that this definition is equivalent to the one that we used in Section 1.

Lemma 2.3. Let $M_{\bullet}^{1} \longleftarrow M_{\bullet}^{2} \longleftarrow M_{\bullet}^{3} \longleftarrow \cdots$ be a projective system of complexes of $R$-modules. Then the projective system of homology modules $H_{i}\left(M_{\bullet}^{1}\right) \longleftarrow$ $H_{i}\left(M_{\bullet}^{2}\right) \longleftarrow H_{i}\left(M_{\bullet}^{3}\right) \longleftarrow \cdots$ is pro-zero if and only if for any injective $R$-module $J$ one has $\lim _{n} H^{i} \operatorname{Hom}_{R}\left(M_{\bullet}^{n}, J\right)=0$.

Proof. This is essentially [30, Lemma 2.4] or [18, Theorem 4.24]. One has $H^{i} \operatorname{Hom}_{R}\left(M_{\bullet}^{n}, J\right) \simeq \operatorname{Hom}_{R}\left(H_{i}\left(M_{\bullet}^{n}\right), J\right)$, so the "only if" assertion is obvious. To prove the "if", pick an injective $R$-module morphism from the $R$-module $H_{i}\left(M_{\bullet}^{l}\right)$ to some injective $R$-module $J$. This morphism represents an element of $H^{i} \operatorname{Hom}_{R}\left(M_{\bullet}^{l}, J\right)$, which has to die in the inductive limit; so there exists $n>k$ such that the composition $H_{i}\left(M_{\bullet}^{n}\right) \longrightarrow H_{i}\left(M_{\bullet}^{l}\right) \longrightarrow J$ is a zero morphism. It follows that the morphism $H_{i}\left(M_{\bullet}^{n}\right) \longrightarrow H_{i}\left(M_{\bullet}^{l}\right)$ is zero.

Example 2.4. It was already mentioned in Section 1 that by [18, Corollary 6.2] the weak proregularity is a property of the radical $\sqrt{I} \subset R$ of the ideal $I$ that a finite sequence $s_{1}, \ldots, s_{m} \in R$ generates and not of the sequence itself. We have also seen that any ideal in a Noetherian commutative ring is weakly proregular. Here is a simple observation providing a class of examples of weakly proregular ideals in non-Noetherian rings [31, Remark 1.14] (cf. [18, Example 4.35 and Theorem 6.5]).

Let $R \longrightarrow T$ be a morphism of commutative rings such that $T$ is a flat module over $R$. Let $\mathbf{s}$ be a finite sequence of elements in the $\operatorname{ring} R$, and let $I$ be the ideal generated by $\mathbf{s}$ in $R$. Then the ideal $T I$ in the $\operatorname{ring} T$ is weakly proregular whenever the ideal $I$ in the ring $R$ is. In other words, the image of a finite sequence $\mathbf{s}$ 
is weakly proregular in $T$ if a sequence $\mathbf{s}$ is weakly proregular in $R$. Indeed, one has $H_{i} \operatorname{Hom}_{T}\left(\operatorname{Tel}_{n}^{\bullet}(T, \mathbf{s}), T\right) \simeq T \otimes_{R} H_{i} \operatorname{Hom}_{R}\left(\operatorname{Tel}_{n}^{\bullet}(R, \mathbf{s}), R\right)$, so for any given $i$ the projective system $H_{i} \operatorname{Hom}_{T}\left(\mathrm{Tel}_{n}^{\bullet}(T, \mathbf{s}), T\right)$ is pro-zero whenever the projective system $H_{i} \operatorname{Hom}_{R}\left(\operatorname{Tel}_{n}^{\bullet}(R, \mathbf{s}), R\right)$ is.

Lemma 2.5. For any weakly proregular finitely generated ideal I in a commutative ring $R$, the restriction of the functor $\Delta_{I}$ to the full subcategory of flat $R$-modules $F$ in $R$-mod is an exact functor isomorphic to the functor of $I$-adic completion $\Lambda_{I}: F \longmapsto$ $\lim _{n} F / I^{n} F$.

Proof. In view of Lemma 2.2(b), this is [18, computations (2-3) in the proof of Theorem 5.21]. The complex $\operatorname{Tel}_{n}^{\bullet}(R, \mathbf{s})$ is homotopy equivalent to the tensor product over $j$ of the two-term complexes of free $R$-modules $\left(R \rightarrow s_{j}^{-n} R\right)$. Therefore, one has $H_{0} \operatorname{Hom}_{R}\left(\operatorname{Tel}_{n}^{\bullet}(R, \mathbf{s}), M\right) \simeq M /\left(\mathbf{s}^{n}\right) M$ for any $R$-module $M$, where $\left(\mathbf{s}^{n}\right)$ denotes the ideal generated by the sequence of elements $s_{1}^{n}, \ldots, s_{m}^{n}$ in $R$.

Clearly, $\lim _{n} M /\left(\mathbf{s}^{n} M\right) \simeq \lim _{n} M / I^{n} M$ for any $R$-module $M$; it remains to check the isomorphism

$$
H_{0}{\underset{\leftarrow}{\longleftarrow}}_{n} \operatorname{Hom}_{R}\left(\operatorname{Tel}_{n}^{\bullet}(R, \mathbf{s}), F\right) \simeq \lim _{n} H_{0} \operatorname{Hom}_{R}\left(\operatorname{Tel}_{n}^{\bullet}(R, \mathbf{s}), F\right)
$$

for a flat $R$-module $F$. Since the terms of the subcomplexes $\operatorname{Tel}_{n}^{\bullet}(R, \mathbf{s})$ are direct summands of the respective terms of the ambient complex $\operatorname{Tel}^{\bullet}(R, \mathbf{s})$, the transition maps in the projective system of complexes $\operatorname{Hom}_{R}\left(\operatorname{Tel}_{n}^{\bullet}(R, \mathbf{s}), M\right)$ are surjective for any $R$-module $M$. Finally, if the projective system $H_{i} \operatorname{Hom}_{R}\left(\operatorname{Tel}_{n}^{\bullet}(R, \mathbf{s}), R\right)$ is pro-zero for every $i>0$, then the projective systems

$$
H_{i} \operatorname{Hom}_{R}\left(\operatorname{Tel}_{n}^{\bullet}(R, \mathbf{s}), F\right) \simeq H_{i} \operatorname{Hom}_{R}\left(\operatorname{Tel}_{n}^{\bullet}(R, \mathbf{s}), R\right) \otimes_{R} F
$$

are also pro-zero for any flat $R$-module $F$ and every $i>0$. Consequently, ${\underset{\lim }{\longleftarrow}}_{n}^{1} H_{i} \operatorname{Hom}_{R}\left(\mathrm{Tel}_{n}^{\bullet}(R, \mathbf{s}), F\right)=0$ for all $i \in \mathbb{Z}$.

Example 2.6. It is clear from the above computation that for any ideal $I$ generated by a finite sequence of elements $\mathbf{s}$ in $R$ and any $R$-module $M$ there is a natural surjective morphism of $R$-modules $\Delta_{I}(M) \longrightarrow \Lambda_{I}(M)$ with the kernel isomorphic to $\lim _{n}^{1} H_{1} \operatorname{Hom}_{R}\left(\operatorname{Tel}_{n}^{\bullet}(R, \mathbf{s}), M\right)$. The following example shows that this kernel may be nontrivial even for the $R$-module $M=R$ when the weak proregularity is not assumed.

Let the sequence $\mathbf{s}$ consist of a single element $s \in R$. Then the homology group $H_{1} \operatorname{Hom}_{R}\left(\operatorname{Tel}_{n}^{\bullet}(R, s), M\right)$ is isomorphic to the submodule of elements annihilated by $s^{n}$ in $M$; for any $n>l$, the map $H_{1} \operatorname{Hom}_{R}\left(\operatorname{Tel}_{n}^{\bullet}(R, s), M\right) \longrightarrow H_{1} \operatorname{Hom}_{R}\left(\operatorname{Tel}_{l}^{\bullet}(R, s), M\right)$ is the multiplication with $s^{n-l}$. Now let $R$ be the commutative algebra over a field $k$ generated by an infinite sequence of elements $x_{1}, x_{2}, x_{3}, \ldots$ and an additional generator $s$ with the relations $x_{i} x_{j}=0$ and $s^{i} x_{i}=0$ for all $i, j \geqslant 1$. Then the submodule of elements annihilated by $s^{n}$ in $R$ is generated by the elements $x_{1}, \ldots, x_{n}, s x_{n+1}$, $s^{2} x_{n+2}, \ldots$, and the map of multiplication with $s^{n-l}$ never annihilates this submodule entirely for $l \geqslant 1$, so the projective system $H_{1} \operatorname{Hom}_{R}\left(\operatorname{Tel}_{n}^{\bullet}(R, s), R\right)$ is not pro-zero and the one-element sequence $s \in R$ is not weakly proregular. 
Furthermore, one can compute the $R$-module $\lim _{n}^{1} H_{1} \operatorname{Hom}_{R}\left(\operatorname{Tel}_{n}^{\bullet}(R, s), R\right)$ as being isomorphic to the module of formal power series $W[[s]]$ in the variable $s$ with the coefficient space $W$ equal to the derived projective limit $\prod_{i} k x_{i} / \bigoplus_{i} k x_{i}$ of the projective system of vector spaces $k x_{n} \oplus k x_{n+1} \oplus \cdots$ and their obvious embeddings. The generators $x_{i} \in R$ act by zero in the $R$-module $W[[s]]$, while the generator $s \in R$ acts in the power series in $s$ in the standard way.

Lemma 2.7. (a) For any weakly proregular finite sequence of elements $\mathbf{s}$ in a commutative ring $R$, the complex $\operatorname{Hom}_{R}\left(\operatorname{Tel}^{\bullet}(R, \mathbf{s}), M\right)$ assigned to an $R$-module $M$ computes the left derived functor $\mathbb{L}_{*} \Delta_{I}(M)$ of the right exact functor $\Delta_{I}$, viewed as taking values in the category $R$-mod.

(b) For any weakly proregular finitely generated ideal $I$ in a commutative ring $R$, (the left derived functor $\mathbb{L}_{*} \Delta_{I}$ of) the right exact functor $\Delta_{I}: R-\bmod \longrightarrow R-\bmod _{I \text {-ctra }}$ has finite homological dimension not exceeding the minimal number of generators of the ideal $I$.

Proof. Part (a): according to [18, Theorem 5.21], one has $H_{i} \operatorname{Hom}_{R}\left(\mathrm{Tel}^{\bullet}(R, \mathbf{s}), F\right)=0$ for any flat $R$-module $F$ and all $i>0$ (we have essentially explained as much already in the proof of Lemma 2.5). Taking also into account Lemma 2.2(b), the argument proceeds from this point in the (standard) way dual to the proof of Lemma 1.2(a) above. As the embedding $R-\bmod _{I \text {-ctra }} \longrightarrow R$-mod is an exact functor taking nonzero objects to nonzero objects, part (b) follows. (Cf. [18, Corollaries 5.25 and 5.27].)

Lemma 2.8. For any weakly proregular finitely generated ideal I in a commutative ring $R$, the derived functor of the functor $\Delta_{I}$ takes $I$-contramodules to themselves, $i$. e., there is a natural isomorphism $\Delta_{I}(P) \simeq P$ and one has $\mathbb{L}_{n} \Delta_{I}(P)=0$ for all $n>0$ and any $I$-contramodule $R$-module $P$.

Proof. This is Lemma 2.2(c) together with Lemma 2.7(a). Notice that the isomorphism $\Delta_{I}(P) \simeq P$ follows already from the fact that the functor $\Delta_{I}$ is left adjoint to the fully faithful embedding functor $R$ - $\bmod _{I \text {-ctra }} \longrightarrow R$-mod. It is only the second assertion of the lemma that depends on the weak proregularity assumption.

Now we are in the position to prove the main result of this section.

Theorem 2.9. Let $R$ be a commutative ring and $I \subset R$ be a weakly proregular finitely generated ideal. Then for any symbol $\star=b,+,-, \varnothing$, abs + , abs - , ctr, or abs, the triangulated functor $\mathrm{D}^{\star}\left(R-\bmod _{I-\mathrm{ctra}}\right) \longrightarrow \mathrm{D}^{\star}(R$-mod) induced by the embedding of abelian categories $R-\bmod _{I \text {-ctra }} \longrightarrow R$-mod is fully faithful.

Proof. The argument is similar to the proof of Theorem 1.3. In the case of a Noetherian ring $R$ and the derived categories $\mathrm{D}^{\mathrm{b}}$ or $\mathrm{D}^{-}$, the assertion follows from [23, Propositions B.9.1 and B.10.1] (see also [26, Proposition 2.2.2(b)]). A proof of the general case proceeds as follows.

Consider the full subcategory $R$-mod $\operatorname{m-ctra-adj}_{C} \subset R$-mod consisting of all the $R$-modules $M$ for which $\mathbb{L}_{n} \Delta_{I}(M)=0$ for all $n>0$. The full subcategory $R$-mod $\operatorname{m-ctra-adj}_{\text {is }}$ closed under extensions, kernels of surjective morphisms, and 
infinite products in $R$-mod; and, according to Lemma $2.7(\mathrm{~b})$, every $R$-module has a finite left resolution of uniformly bounded length by objects from $R$ - $\bmod _{I \text {-ctra-adj}}$. Hence the subcategory $R$ - $\bmod _{I \text {-ctra-adj }}$ inherits the exact category structure of the abelian category $R$-mod, and for any symbol $\star$ on our list the triangulated functor $\mathrm{D}^{\star}\left(R-\bmod _{I \text {-ctra-adj }}\right) \longrightarrow \mathrm{D}^{\star}(R-$ mod $)$ is an equivalence of triangulated categories [24, Proposition A.5.6],

$$
\mathrm{D}^{\star}\left(R-\bmod _{I \text {-ctra-adj }}\right) \simeq \mathrm{D}^{\star}(R-\bmod ) .
$$

Obviously, the restriction of the functor $\Delta_{I}$ to the full exact subcategory

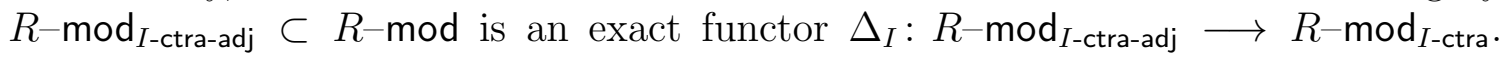
Applying the functor $\Delta_{I}$ to complexes of modules from the category $R-\bmod _{I \text {-ctra-adj }}$ termwise, we obtain the left derived functor

$$
\mathbb{L} \Delta_{I}: \mathrm{D}^{\star}(R-\bmod ) \longrightarrow \mathrm{D}^{\star}\left(R-\bmod _{I \text {-ctra }}\right) .
$$

The derived functor $\mathbb{L} \Delta_{I}$ is left adjoint to the triangulated functor $\mathrm{D}^{\star}\left(R-\bmod _{I \text {-ctra }}\right)$ $\longrightarrow \mathrm{D}^{\star}(R-$ mod $)$ induced by the embedding of abelian categories $R$ - $\bmod _{I \text {-ctra }} \longrightarrow$ $R-\bmod [21$, Lemma 8.3].

Furthermore, the abelian subcategory $R$ - $\bmod _{I \text {-ctra }} \subset R$-mod is contained in the exact subcategory $R-\bmod _{I \text {-ctra-adj }}$,

$$
R-\bmod _{I \text {-ctra }} \subset R-\bmod _{I \text {-ctra-adj }}
$$

by Lemma 2.8 , and the functor $\Delta_{I}$ takes $I$-contramodule $R$-modules to themselves. Therefore, the composition of adjoint functors

$$
\mathrm{D}^{\star}\left(R-\bmod _{I-\text { ctra }}\right) \longrightarrow \mathrm{D}^{\star}(R-\bmod ) \longrightarrow \mathrm{D}^{\star}\left(R-\bmod _{I-\text { ctra }}\right)
$$

is isomorphic to the identity functor on $\mathrm{D}^{\star}\left(R-\bmod _{I \text {-ctra }}\right)$. It follows immediately that the functor $\mathrm{D}^{\star}\left(R-\bmod _{I \text {-ctra }}\right) \longrightarrow \mathrm{D}^{\star}(R-\bmod )$ is fully faithful, while the functor $\mathbb{L} \Delta_{I}: \mathrm{D}^{\star}(R-\bmod ) \longrightarrow \mathrm{D}^{\star}\left(R-\bmod _{I \text {-ctra }}\right)$ is a Verdier quotient functor.

Corollary 2.10. Let $R$ be a commutative ring and $I \subset R$ be a weakly proregular finitely generated ideal. Then for any symbol $\star=\mathrm{b},+,-$, or $\varnothing$, the triangulated functor $\mathrm{D}^{\star}\left(R-\bmod _{I \text {-ctra }}\right) \longrightarrow \mathrm{D}^{\star}(R$-mod $)$ identifies the derived category $\mathrm{D}^{\star}\left(R\right.$ - $\left.\bmod _{I \text {-ctra }}\right)$ with the full subcategory $\mathrm{D}_{I-\mathrm{ctra}}^{\star}(R-\mathrm{mod}) \subset \mathrm{D}^{\star}(R-\mathrm{mod})$ consisting of all the complexes with the cohomology modules belonging to $R-\bmod _{I \text {-ctra }}$.

Proof. The proof is similar to that of Corollary 1.4. It is obvious that the image of the functor $\mathrm{D}^{\star}\left(R-\bmod _{I \text {-ctra }}\right) \longrightarrow \mathrm{D}^{\star}(R-$ mod $)$ is contained in $\mathrm{D}_{I \text {-ctra }}^{\star}(R$-mod $)$. To prove the converse inclusion, notice that, according to the proof of Theorem 2.9, the image of the fully faithful functor $\mathrm{D}^{\star}\left(R-\bmod _{I \text {-ctra }}\right) \longrightarrow \mathrm{D}^{\star}(R-$ mod $)$ consists precisely of all the complexes fixed by the composition of adjoint functors $\mathrm{D}^{\star}(R-$ mod $) \longrightarrow$ $\mathrm{D}^{\star}\left(R-\bmod _{I \text {-ctra }}\right) \longrightarrow \mathrm{D}^{\star}(R$-mod $)$. Since the functor $\mathbb{L} \Delta_{I}$ has finite homological dimension, by the way-out functor argument of [8, Proposition I.7.1] a complex viewed as an object of the conventional derived category is fixed by this composition whenever its cohomology modules are. 


\section{MGM Duality Theorem}

Let $R$ be a commutative ring and $\mathbf{s}$ be a finite sequence of its elements $s_{1}, \ldots$, $s_{m} \in R$. We recall the constructions of complexes $C_{\mathbf{s}}(R)$ and $C_{\mathbf{s}}(R)^{\sim}$ from Section 1 .

Lemma 3.1. The tensor product complex $C_{\mathbf{s}}^{\bullet}(R) \otimes_{R} C_{\mathbf{s}}(R)^{\sim}$ is a contractible complex of $R$-modules.

Proof. It suffices to show that for every term $C_{\mathbf{s}}^{i}(R)$ of the complex $C_{\mathbf{s}}(R)$ the tensor product $C_{\mathbf{s}}^{i}(R) \otimes_{R} C_{\mathbf{s}}^{\bullet}(R)^{\sim}$ is a contractible complex, because the complex $C_{\mathbf{s}}^{\bullet}(R) \otimes_{R} C_{\mathbf{s}} \cdot(R)^{\sim}$ can be obtained from the complexes $C_{\mathbf{s}}^{i}(R) \otimes_{R} C_{\mathbf{s}}(R)^{\sim}$ by iterating the operations of shift and cone in the homotopy category of complexes of $R$-modules. Hence it is enough to check that the complex $C_{\mathbf{s}}^{\bullet}(R)^{\sim}\left[s_{j}^{-1}\right]$ is contractible for every $j$. This was already done in the proof of Lemma 1.1(a).

Following [18, Section 8], let us endow the complex $C_{\mathbf{s}}^{\bullet}(R)$ with the Čech/singular cochain multiplication, making it a (noncommutative) DG-ring. The natural morphism of complexes $k: R \longrightarrow C_{\mathbf{s}}^{\bullet}(R)$ is a morphism of DG-rings with the image lying in the center of $C_{\mathbf{s}}^{\bullet}(R)$, making $C_{\mathbf{s}}^{\bullet}(R)$ a DG-algebra over $R$. The following lemma is our version of [18, Lemmas 4.29 and 7.9].

Lemma 3.2. (a) The two morphisms of complexes

$$
C_{\mathbf{s}}^{\bullet}(R)^{\sim} \otimes_{R} C_{\mathbf{s}}^{\bullet}(R)^{\sim} \rightrightarrows C_{\mathbf{s}}^{\bullet}(R)^{\sim}
$$

induced by the natural morphism $C_{\mathbf{s}}^{\bullet}(R)^{\sim} \longrightarrow R$ are homotopy equivalences of complexes of R-modules.

(b) The three morphisms of complexes

$$
C_{\mathbf{s}}^{\bullet}(R) \rightrightarrows C_{\mathbf{s}}^{\bullet}(R) \otimes_{R} C_{\mathbf{s}}^{\bullet}(R) \longrightarrow C_{\mathbf{s}}^{\bullet}(R)
$$

provided by the unit and multiplication in the $D G$-algebra $C_{\mathbf{s}}(R)$ are homotopy equivalences of complexes of $R$-modules.

Proof. Follows immediately from Lemma 3.1.

Our next goal is to rewrite the geometric arguments of Section 0.5 in the algebraic language. A key ingredient is the derived category $\mathrm{D}^{\star}\left(C_{\mathbf{s}}^{\bullet}(R)\right.$-mod) of left DG-modules over the DG-ring $C_{\mathbf{s}}^{\bullet}(R)$, which is used in lieu of the derived category $\mathrm{D}^{\star}(U-\mathrm{qcoh}) \simeq \mathrm{D}^{\star}(U-\mathrm{ctrh})$ of quasi-coherent sheaves/contraherent cosheaves on the open subscheme $U=X \backslash Z$, where $X=\operatorname{Spec} R$ and $Z=\operatorname{Spec} R / I$.

The DG-ring $C_{\mathbf{s}}(R)$ being positively cohomologically graded, derived categories of the second kind of DG-modules over it may differ from the conventional derived categories even for bounded DG-modules (cf. [22, Section 3.4, last paragraph of Section 0.4, and Example 6.6]). So we restrict our exposition to the four conventional derived categories $\mathrm{D}^{\mathrm{b}}, \mathrm{D}^{+}, \mathrm{D}^{-}$, and $\mathrm{D}$. Notice that some, though not all, of the related geometric results of [24, Sections 4.6 and 4.8] are applicable to the absolute derived categories $\mathrm{D}^{\text {abs }}, \mathrm{D}^{\text {abs- }}$, and $\mathrm{D}^{\text {abs }}$ as well. 
So let $\star$ be one of the conventional derived category symbols $b,+,-$, or $\varnothing$. The derived categories $\mathrm{D}^{\star}\left(C_{\mathbf{s}}^{\bullet}(R)\right.$-mod) are defined by inverting the classes of quasiisomorphisms in the homotopy categories of (respectively bounded) left DG-modules over $C_{\mathbf{s}}^{\bullet}(R)$. Denote by $k_{*}: \mathrm{D}^{\star}\left(C_{\mathbf{s}}^{\bullet}(R)-\bmod \right) \longrightarrow \mathrm{D}^{\star}(R-\bmod )$ the functor of restriction of scalars with respect to the DG-ring morphism $k: R \longrightarrow C_{\mathbf{s}}^{\bullet}(R)$.

Proposition 3.3. (a) The triangulated functor $k_{*}$ has a left adjoint functor $k^{*}: \mathrm{D}^{\star}(R-$ mod $) \longrightarrow \mathrm{D}^{\star}\left(C_{\mathbf{s}}^{\bullet}(R)-\bmod \right)$ and a right adjoint functor $\mathbb{R} k^{!}: \mathrm{D}^{\star}(R$-mod $)$ $\longrightarrow \mathrm{D}^{\star}\left(C_{\mathbf{s}}^{\bullet}(R)-\right.$ mod $)$.

(b) The compositions $k^{*} \circ k_{*}$ and $\mathbb{R} k^{!} \circ k_{*}$ are isomorphic to the identity functors on the category $\mathrm{D}^{\star}\left(C_{\mathbf{s}}^{\bullet}(R)\right.$-mod), functor $k_{*}$ is fully faithful, and the functors $k^{*}$ and $\mathbb{R} k^{!}$ are Verdier quotient functors.

Proof. Part (a): the functor $k^{*}$ is easy to define, as $C_{\mathbf{s}}^{\bullet}(R)$ is a finite complex of flat $R$-modules, so setting $k^{*}\left(M^{\bullet}\right)=C_{\mathbf{s}}^{\bullet}(R) \otimes_{R} M^{\bullet}$ suffices for any symbol $\star$. In the case of $\star=\varnothing$, both the adjoint functors $\mathbb{L} f^{*}$ and $\mathbb{R} f^{!}$exist for any morphism of DG-rings $f$ [22, Section 1.7]. To construct the derived functor $\mathbb{R} k$ ! for bounded derived categories $\mathrm{D}^{\star}$, notice that $C_{\mathbf{s}}(R)$ is a finite complex of $R$-modules of projective dimension at most 1 , so embedding a complex of $R$-modules $M^{\bullet}$ into a complex of injective $R$-modules $J^{\bullet}$, replacing $M^{\bullet}$ with the cocone $N^{\bullet}$ of the morphism $J^{\bullet} \longrightarrow$ $J^{\bullet} / M^{\bullet}$ and setting $\mathbb{R} k^{!}\left(M^{\bullet}\right)=\operatorname{Hom}_{R}\left(C_{\mathbf{s}}^{\bullet}(R), N^{\bullet}\right)$ does the job. These constructions of the functors $k_{*}, k^{*}$, and $\mathbb{R} k^{!}$are actually applicable to the derived categories with exotic symbols $\star=$ abs + , abs - , or abs just as well. The constructions of the functors $k_{*}$ and $k^{*}$ also work for the coderived categories, while the constructions of the functors $k_{*}$ and $\mathbb{R} k^{!}$are applicable to the contraderived categories.

To prove part (b), notice that by Lemma 3.2(b) the adjunction morphisms

$$
k_{*} k^{*} \rightrightarrows k_{*} k^{*} k_{*} k^{*} \longrightarrow k_{*} k^{*}
$$

are isomorphisms of functors on the category $\mathrm{D}^{\star}(R-\mathrm{mod}), \quad k_{*} k^{*} \simeq k_{*} k^{*} k_{*} k^{*}$. It follows that for any objects $M^{\bullet}$ and $N^{\bullet} \in \mathrm{D}^{\star}(R$-mod) the adjunction morphisms $k^{*} N^{\bullet} \longrightarrow k^{*} k_{*} k^{*} N^{\bullet} \longrightarrow k^{*} N^{\bullet}$ induce isomorphisms of Hom modules

$$
\operatorname{Hom}_{\mathrm{D}}\left(k^{*} M^{\bullet}, k^{*} N^{\bullet}\right) \longrightarrow \operatorname{Hom}_{\mathrm{D}}\left(k^{*} M^{\bullet}, k^{*} k_{*} k^{*} N^{\bullet}\right) \longrightarrow \operatorname{Hom}_{\mathrm{D}}\left(k^{*} M^{\bullet}, k^{*} N^{\bullet}\right)
$$

in the derived category $\mathrm{D}^{\star}\left(C_{\mathbf{s}}^{\bullet}(R)\right.$-mod). In other words, the adjunction morphisms induce isomorphisms of the functors represented by the objects $k^{*} N^{\bullet}$ and $k^{*} k_{*} k^{*} N^{\bullet}$ on the essential image of the functor $k^{*}$ (viewed as a full subcategory in $\mathrm{D}^{\star}\left(C_{\mathbf{s}}^{\bullet}(R)-\bmod \right)$ ). As the objects $k^{*} N^{\bullet}$ and $k^{*} k_{*} k^{*} N^{\bullet}$ also belong to this essential image, it follows that the adjunction morphisms

$$
k^{*} N^{\bullet} \longrightarrow k^{*} k_{*} k^{*} N^{\bullet} \longrightarrow k^{*} N^{\bullet}
$$

are isomorphisms of functors, $k^{*} \simeq k^{*} k_{*} k^{*}$. Hence the adjunction morphisms

$$
k^{*} k_{*} \longrightarrow k^{*} k_{*} k^{*} k_{*} \rightrightarrows k^{*} k_{*}
$$

are also isomorphisms. Applying the same argument with the roles of the two categories $\mathrm{D}^{\star}(R$-mod $)$ and $\mathrm{D}^{\star}\left(C_{\mathbf{s}}^{\bullet}(R)\right.$-mod $)$ switched, one can see that the adjunction 
morphisms

$$
k_{*} \longrightarrow k_{*} k^{*} k_{*} \longrightarrow k_{*}
$$

are also isomorphisms of functors, $k_{*} \simeq k_{*} k^{*} k_{*}$.

Finally, we observe that the restrictions of scalars are conservative functors between conventional derived categories, i. e., a morphism of DG-modules over $C_{\mathbf{s}}^{\bullet}(R)$ is a quasi-isomorphism whenever it is a quasi-isomorphism of DG-modules over $R$ (cf. [21, Remark 8.4.3 and Section 8.4.4] for a discussion of the conservativity problem for functors between derived categories of the second kind). The functor $k_{*}$ being conservative and the natural morphism $k_{*} k^{*} k_{*} B^{\bullet} \longrightarrow k_{*} B^{\bullet}$ being an isomorphism in $\mathrm{D}^{\star}\left(R\right.$-mod) for any DG-module $B^{\bullet} \in \mathrm{D}^{\star}\left(C_{\mathbf{s}}^{\bullet}(R)-\right.$ mod $)$, we can conclude that the morphism $k^{*} k_{*} B^{\bullet} \longrightarrow B^{\bullet}$ is an isomorphism in $\mathrm{D}^{\star}\left(C_{\mathbf{s}}^{\bullet}(R)\right.$-mod).

Hence it follows that the functor $k_{*}$ is fully faithful, the functors $k^{*}$ and $\mathbb{R} k^{!}$are Verdier quotient functors, and the composition $\mathbb{R} k^{!} \circ k_{*}$ is isomorphic to the identity functor. Alternatively, the latter assertion can be proven directly in the way similar to the above argument.

Now we are ready to prove our version of the MGM duality theorem for commutative rings with finitely generated ideals. For any commutative ring $R$ with an ideal $I$ generated by a finite sequence of elements $s_{1}, \ldots, s_{m} \in R$, consider the full subcategory of complexes with $I$-torsion cohomology modules

$$
\mathrm{D}_{I \text {-tors }}^{\star}(R \text {-mod }) \subset \mathrm{D}^{\star}(R-\text { mod })
$$

and the full subcategory of complexes with $I$-contramodule cohomology modules

$$
\mathrm{D}_{I-\text { ctra }}^{\star}(R-\text { mod }) \subset \mathrm{D}^{\star}(R-\text { mod })
$$

in the derived category of $R$-modules. The essential image of the fully faithful functor $k_{*}$ from Proposition 3.3

$$
k_{*} \mathrm{D}^{\star}\left(C_{\mathbf{s}}^{\bullet}(R)-\bmod \right) \subset \mathrm{D}^{\star}(R-\bmod )
$$

is a third triangulated subcategory in $\mathrm{D}^{\star}(R-$ mod $)$ that is of interest to us.

Theorem 3.4. The subcategory $k_{*} \mathrm{D}^{\star}\left(C_{\mathbf{s}}(R)\right.$-mod) is the right orthogonal complement to the subcategory $\mathrm{D}_{I \text {-tors }}^{\star}(R$-mod) and the left orthogonal complement to the subcategory $\mathrm{D}_{I-\text { ctra }}^{\star}(R$-mod $)$ in $\mathrm{D}^{\star}(R$-mod). The passage to the Verdier quotient category by the triangulated subcategory $k_{*} \mathrm{D}^{\star}\left(C_{\mathbf{s}}^{\bullet}(R)\right.$-mod $) \subset \mathrm{D}^{\star}(R$-mod) establishes an equivalence between the triangulated categories $\mathrm{D}_{I \text {-tors }}^{\star}(R$-mod $)$ and $\mathrm{D}_{I \text {-ctra }}^{\star}(R$-mod $)$,

$$
\mathrm{D}_{I \text {-tors }}^{\star}(R \text {-mod }) \simeq \mathrm{D}^{\star}(R \text {-mod }) / k_{*} \mathrm{D}^{\star}\left(C_{\mathbf{s}}^{\bullet}(R)-\text { mod }\right) \simeq \mathrm{D}_{I \text {-ctra }}^{\star}(R \text {-mod }) .
$$

The assertion of the theorem can be equivalently restated as the existence of two semiorthogonal decompositions of the category $\mathrm{D}^{\star}(R$-mod), one of them formed by the two full subcategories $k_{*} \mathrm{D}^{\star}\left(C_{\mathbf{s}}^{\bullet}(R)\right.$-mod $)$ and $\mathrm{D}_{I \text {-tors }}^{\star}(R$-mod $) \subset \mathrm{D}^{\star}(R$-mod $)$, and the other one consisting of the two full subcategories $\mathrm{D}_{I \text {-ctra }}^{\star}(R$-mod) and $k_{*} \mathrm{D}^{\star}\left(C_{\mathbf{s}}^{\bullet}(R)-\right.$ mod) in $\mathrm{D}^{\star}(R-$ mod) (see, e. g., [22, Section 1.3]). 
Proof. It is clear from Proposition 3.3 that the passage to the quotient category by the image of the functor $k_{*}$ establishes an equivalence between the kernels of the functors $k^{*}$ and $\mathbb{R} k^{!}$,

$$
\operatorname{ker}\left(k^{*}\right) \simeq \mathrm{D}^{\star}(R-\bmod ) / \operatorname{im} k_{*} \simeq \operatorname{ker}\left(\mathbb{R} k^{!}\right) .
$$

In other words, we have two semiorthogonal decompositions, one of them formed by the two full subcategories $\operatorname{im}\left(k_{*}\right)$ and $\operatorname{ker}\left(k^{*}\right)$, and the other one by the two full subcategories $\operatorname{ker}\left(\mathbb{R} k^{!}\right)$and $\operatorname{im}\left(k_{*}\right)$, in the derived category $\mathrm{D}^{\star}(R$-mod $)$. To prove the theorem, we only have to identify the full subcategory $\operatorname{ker}\left(k^{*}\right)$ with the full subcategory $\mathrm{D}_{I \text {-tors }}^{\star}(R$-mod $) \subset \mathrm{D}^{\star}(R$-mod $)$ and the full subcategory $\operatorname{ker}\left(\mathbb{R} k^{!}\right)$with the full subcategory $\mathrm{D}_{I \text {-ctra }}^{\star}(R-\bmod ) \subset \mathrm{D}^{\star}(R-\bmod )$.

As it always happens with semiorthogonal decompositions, the adjunction morphisms id $\longrightarrow k_{*} k^{*}$ and $k_{*} \mathbb{R} k^{!} \longrightarrow$ id have functorial cones. The subcategory $\operatorname{ker}\left(k^{*}\right) \subset \mathrm{D}^{\star}(R-\bmod )$ coincides with the image of the functor

$$
\text { cocone }\left(\mathrm{id} \rightarrow k_{*} k^{*}\right): \mathrm{D}^{\star}(R-\bmod ) \longrightarrow \mathrm{D}^{\star}(R-\bmod ),
$$

while the subcategory $\operatorname{ker}\left(\mathbb{R} k^{!}\right) \subset \mathrm{D}^{\star}(R$-mod $)$ coincides with the image of the functor

$$
\operatorname{cone}\left(k_{*} \mathbb{R} k^{!} \rightarrow \mathrm{id}\right): \mathrm{D}^{\star}(R-\bmod ) \longrightarrow \mathrm{D}^{\star}(R-\bmod ) .
$$

Just as the functors $k_{*} k^{*}$ and $k_{*} \mathbb{R} k^{!}$, both the functors cocone(id $\rightarrow k_{*} k^{*}$ ) and $\operatorname{cone}\left(k_{*} \mathbb{R} k^{!} \rightarrow \mathrm{id}\right)$ are projectors on their respective images: each of them is naturally isomorphic to its composition with itself. Moreover, there are natural transformations

$$
\text { cocone }\left(\mathrm{id} \rightarrow k_{*} k^{*}\right) \longrightarrow \text { id } \quad \text { and } \quad \mathrm{id} \longrightarrow \operatorname{cone}\left(k_{*} \mathbb{R} k^{!} \rightarrow \mathrm{id}\right) \text {. }
$$

A complex $M^{\bullet} \in \mathrm{D}^{\star}(R$-mod $)$ belongs to the subcategory $\operatorname{ker}\left(k^{*}\right)$ if and only if the morphism cocone $\left(\mathrm{id} \rightarrow k_{*} k^{*}\right)\left(M^{\bullet}\right) \longrightarrow M^{\bullet}$ is a quasi-isomorphism, while a complex $P^{\bullet} \in \mathrm{D}^{\star}\left(R\right.$-mod) belongs to the subcategory $\operatorname{ker}\left(\mathbb{R} k^{!}\right)$if and only if the morphism $P^{\bullet} \longrightarrow \operatorname{cone}\left(k_{*} \mathbb{R} k^{!} \rightarrow \mathrm{id}\right)\left(P^{\bullet}\right)$ is a quasi-isomorphism.

In view of the constructions of the functors $k^{*}$ and $\mathbb{R} k^{!}$in the proof of part (a) of the proposition, the functor cocone(id $\left.\rightarrow k_{*} k^{*}\right)$ is isomorphic to the functor of tensor product with the complex $C_{\mathbf{s}}^{\bullet}(R)^{\sim}$,

$$
C_{\mathbf{s}}^{\bullet}(R)^{\sim} \otimes_{R}-: \mathrm{D}^{\star}(R-\bmod ) \longrightarrow \mathrm{D}^{\star}(R-\bmod ),
$$

while the functor cone $\left(k_{*} \mathbb{R} k^{!} \rightarrow\right.$ id $)$ is the functor of right derived homomorphisms from $C_{\mathbf{s}}(R)^{\sim}$. The latter can be easily computed as the homomorphisms from the complex of free $R$-modules $\operatorname{Tel}^{\bullet}(R, \mathbf{s})$,

$$
\operatorname{Hom}_{R}\left(\mathrm{Tel}^{\bullet}(R, \mathbf{s}),-\right): \mathrm{D}^{\star}(R-\bmod ) \longrightarrow \mathrm{D}^{\star}(R-\bmod ) .
$$

To sum up, a complex $M^{\bullet} \in \mathrm{D}^{\star}\left(R\right.$-mod) belongs to the subcategory $\operatorname{ker}\left(k^{*}\right)$ if and only if the natural map $C_{\mathbf{s}}^{\bullet}(R)^{\sim} \otimes_{R} M^{\bullet} \longrightarrow M^{\bullet}$ is a quasi-isomorphism, while a complex $P^{\bullet} \in \mathrm{D}^{\star}\left(R\right.$-mod) belongs to the subcategory $\operatorname{ker}\left(\mathbb{R} k^{!}\right)$if and only if the natural map $P^{\bullet} \longrightarrow \operatorname{Hom}_{R}\left(\mathrm{Tel}^{\bullet}(R, \mathbf{s}), P^{\bullet}\right)$ is a quasi-isomorphism.

Finally, we recall that $C_{\mathbf{s}}^{\bullet}(R)^{\sim}$ and $\mathrm{Tel}^{\bullet}(R, \mathbf{s})$ are finite complexes of $R$-modules, so Hartshorne's way-out functor argument of [8, Propositions I.7.1 and I.7.3] applies. Hence the cohomology modules of the complex $C_{\mathbf{s}}^{\bullet}(R)^{\sim} \otimes_{R} M^{\bullet}$ are $I$-torsion 
$R$-modules for any complex of $R$-modules $M^{\bullet}$ by Lemma 1.1(a). Similarly, the cohomology modules of the complex $\operatorname{Hom}_{R}\left(\mathrm{Tel}^{\bullet}(R, \mathbf{s}), M^{\bullet}\right)$ are $I$-contramodule $R$-modules for any complex of $R$-modules $M^{\bullet}$ by Lemma 2.2(a). We have shown that the cohomology modules of any complex $M^{\bullet} \in \operatorname{ker}\left(k^{*}\right)$ are $I$-torsion $R$-modules, and the cohomology modules of any complex $P^{\bullet} \in \operatorname{ker}\left(\mathbb{R} k^{!}\right)$are $I$-contramodule $R$-modules.

Conversely, it follows from the above that a complex of $R$-modules belongs to the subcategory $\operatorname{ker}\left(k^{*}\right)$ or $\operatorname{ker}\left(\mathbb{R} k^{!}\right)$whenever its cohomology modules, viewed as oneterm complexes, do. When $M$ is an $I$-torsion $R$-module, the map $C_{\mathbf{s}}^{\bullet}(R)^{\sim} \otimes_{R} M \longrightarrow$ $M$ is a quasi-isomorphism by Lemma $1.1(\mathrm{c})$. When $P$ is an $I$-contramodule, the map $P \longrightarrow \operatorname{Hom}_{R}\left(T^{\bullet}(R, \mathbf{s}), P\right)$ is a quasi-isomorphism by Lemma 2.2(c). Therefore, the full subcategory $\operatorname{ker}\left(k^{*}\right) \subset \mathrm{D}^{\star}(R$-mod) consists precisely of all the complexes with $I$-torsion cohomology modules, and the full subcategory $\operatorname{ker}\left(\mathbb{R} k^{!}\right) \subset \mathrm{D}^{\star}(R$-mod) consists precisely of all the complexes with $I$-contramodule cohomology modules.

Notice that no weak proregularity assumption has been used in this proof (cf. [6, Propositions 6.12 and 6.15]).

When the ideal $I \subset R$ is weakly proregular, the MGM duality theorem takes the form promised in the formula (2) in Section 0.9 of the introduction.

Corollary 3.5. Let $R$ be a commutative ring and $I \subset R$ be a weakly proregular finitely generated ideal. Then for any symbol $\star=\mathrm{b},+,-$, or $\varnothing$, the (respectively bounded or unbounded) derived categories $\mathrm{D}^{\star}\left(R-\bmod _{I \text {-tors }}\right)$ and $\mathrm{D}^{\star}\left(R\right.$ - $\left.\bmod _{I \text {-ctra }}\right)$ are naturally equivalent,

$$
\mathrm{D}^{\star}\left(R-\bmod _{I \text {-tors }}\right) \simeq \mathrm{D}^{\star}\left(R-\bmod _{I \text {-ctra }}\right) .
$$

Proof. Compare the results of Corollaries 1.4 and 2.10, and Theorem 3.4. Another proof of this equivalence of derived categories (in somewhat greater generality) will be obtained in Theorem 5.10 below.

\section{Dedualizing Complexes for Ideals with Artinian Quotient Rings}

Let $R$ be a Noetherian commutative ring and $I \subset R$ be an ideal such that the quotient ring $R / I$ is Artinian. (One would lose essentially no generality by assuming that $I$ is a maximal ideal in $R$.) Fix an injective envelope of the $R$-module $R / I$ and denote it by $C$. Then $C$ is an injective $I$-torsion $R$-module.

For any $R$-module $M$ and an integer $n \geqslant 1$, we denote by ${ }_{n} M$ the submodule of all elements annihilated by $I^{n}$ in $M$. In particular, ${ }_{n} C$ is a finitely generated $R / I^{n}$-module and an injective cogenerator of the abelian category $R / I^{n}$-mod. The following lemma is standard [13].

Lemma 4.1. Let $M$ be an I-torsion $R$-module. Then the following conditions are equivalent:

(1) the $R / I$-module ${ }_{1} M$ is finitely generated;

(2) for every $n \geqslant 1$, the $R / I^{n}$-module ${ }_{n} M$ is finitely generated; 
(3) $M$ is a submodule of a finite direct sum of copies of $C$;

(4) $M$ is an Artinian $R$-module.

Given a derived category symbol $\star=b,+,-, \varnothing$, abs + , abs - , or abs, we denote by $\mathrm{D}^{\star}\left(R-\bmod _{I \text {-tors }}\right)$ and $\mathrm{D}^{\star}\left(R-\bmod _{I \text {-ctra }}\right)$ the corresponding (conventional or absolute) derived categories of the abelian categories $R-\bmod _{I \text {-tors }}$ and $R-\bmod _{I \text {-ctra }}$ of $I$-torsion and $I$-contramodule $R$-modules (see Appendix A for the definitions).

Given an $R$-module $M$ and a set $X$, we denote by $M[X]$ the $X$-indexed direct sum of copies of $M$. The $R$-module $C[X]$ is called the cofree $I$-torsion $R$-module cogenerated by the set $X$ (cf. [23, Sections 1.4 and 1.9$]$ ). The abelian category $R-\bmod _{I \text {-tors }}$ has enough injective objects, which are precisely the direct summands of cofree $I$-torsion $R$-modules. Every injective object of $R$ - $\bmod _{I \text {-tors }}$ is also injective in $R$-mod.

Identifying the bounded below derived category $\mathrm{D}^{+}\left(R-\bmod _{I \text {-tors }}\right)$ with the homotopy category of injective $I$-torsion $R$-modules $\operatorname{Hot}^{+}(R$-mod $I$-tors $)$ and applying the functor $M \longmapsto{ }_{n} M=\operatorname{Hom}_{R}\left(R / I^{n}, M\right)$ to complexes of injective $I$-torsion $R$-modules termwise, we obtain the right derived functor

$$
M^{\bullet} \longmapsto{ }_{n}^{\mathbb{R}} M^{\bullet}: \mathrm{D}^{+}\left(R-\bmod _{I \text {-tors }}\right) \longrightarrow \mathrm{D}^{+}\left(R / I^{n}-\bmod \right) .
$$

The full subcategory of $I$-torsion $R$-modules satisfying the equivalent conditions of Lemma 4.1 is closed under the passages to subobjects, quotient objects, and extensions in $R-\bmod _{I \text {-tors }}$. So the category of Artinian $I$-torsion $R$-modules is abelian. Notice that this abelian category has enough injective objects, which are precisely the direct summands of finitely cogenerated cofree $I$-torsion $R$-modules (i. e., of the finite direct sums of copies of $C$ ).

Lemma 4.2. Fix $n \geqslant 1$. Then a complex $L^{\bullet} \in \mathrm{D}^{+}\left(R\right.$ - $\left.\bmod _{I \text {-tors }}\right)$ has Artinian

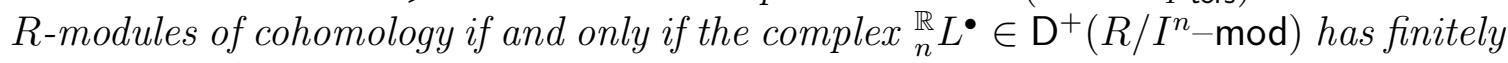
generated $R / I^{n}$-modules of cohomology.

Proof. First one applies Lemma 4.1 in order to prove that the complex ${ }_{n}^{\mathbb{R}} L$ has finitely generated cohomology $R / I^{n}$-modules for every Artinian $I$-torsion $R$-module $L$ (viewed as a one-term complex). In the general case, both the "if" and "only if" assertions are then deduced by induction in the cohomological degree.

Lemma 4.3. Let $L^{\bullet}$ be a bounded below complex of I-torsion $R$-modules with Artinian cohomology modules. Then there exists a bounded below complex of finitely cogenerated cofree $I$-torsion $R$-modules $J^{\bullet}$ together with a quasi-isomorphism of complexes of $I$-torsion $R$-modules $L^{\bullet} \longrightarrow J^{\bullet}$.

Proof. This is a standard step-by-step construction (cf. [27, Lemma 1.2] and the proof of Lemma B.1(c) below).

Let $\mathfrak{R}=\lim _{\leftarrow} R / I^{n}$ denote the $I$-adic completion of the ring $R$. So $\mathfrak{R}$ is a finite direct sum of complete Noetherian local rings. For any set $X$, we put $\mathfrak{R}[[X]]=$ $\lim _{\longleftarrow} R / I^{n}[X]$. For every $n \geqslant 1$, the natural map $R / I^{n} \longrightarrow \operatorname{Hom}_{R / I^{n}}\left({ }_{n} C,{ }_{n} C\right)$ is an isomorphism. Hence one has $\mathfrak{R} \simeq \operatorname{Hom}_{R}(C, C)$, and moreover, $\mathfrak{R}[[X]] \simeq$ $\operatorname{Hom}_{R}(C, C[X])$ for any set $X$. 
Clearly, $\mathfrak{R}[[X]]$ is an $I$-contramodule $R$-module; we call it the free $I$-contramodule $R$-module generated by the set $X$. For any $I$-contramodule $R$-module $P$, one has $\operatorname{Hom}_{R}(\mathfrak{R}[[X]], P)=P^{X}$. There are enough projective objects in $R$ - $\bmod _{I \text {-ctra }}$, and an $I$-contramodule $R$-module is a projective object in $R$ - $\bmod _{I \text {-ctra }}$ if and only if it is a direct summand of a free $I$-contramodule $R$-module (see Lemma 5.3(a) below and the discussion in the two paragraphs preceding it).

Lemma 4.4. Let $P$ be an $I$-contramodule $R$-module. Then the following conditions are equivalent:

(1) the $R / I$-module $P / I P$ is finitely generated;

(2) for every $n \geqslant 1$, the $R / I^{n}$-module $P / I^{n} P$ is finitely generated;

(3) $P$ is a quotient module of a finite direct sum of copies of $\mathfrak{R}$;

(4) $P$ is a Noetherian object of the category of I-contramodule R-modules.

Proof. This lemma looks pretty much like the dual result to Lemma 4.1, but in fact holds in the greater generality of an arbitrary ideal $I$ in a Noetherian commutative ring $R$ (cf. [20, Theorem 0.2]). The implications $(3) \Longrightarrow(2) \Longrightarrow(1)$ are obvious, and $(4) \Longrightarrow(3)$ is easily deduced from the existence of a surjective $R$-module morphism $\mathfrak{R}[[X]] \longrightarrow P$. To prove $(1) \Longrightarrow(3)$, one chooses a finite set of generators $X$ in the $R / I$-module $P / I P$, lifts the composition of morphisms $\mathfrak{R}[[X]] \longrightarrow$ $R / I[X] \longrightarrow P / I P$ to an $R$-module morphism $f: \mathfrak{R}[X]=\mathfrak{R}[[X]] \longrightarrow P$, notices that the cokernel $L$ of the morphism $f$ satisfies $L / I L=0$, and applies the contramodule Nakayama lemma [23, Lemma 1.3.1] together the isomorphism of contramodule categories from [23, Theorem B.1.1]. It also follows from the latter theorem that any $I$-contramodule $R$-module has a natural $\mathfrak{R}$-module structure, and all morphisms between $I$-contramodule $R$-modules are $\mathfrak{R}$-linear. So the implication $(3) \Longrightarrow(4)$ holds because $\mathfrak{R}$ is a Noetherian ring.

Let us call an $I$-contramodule $R$-module finitely generated if it satisfies the equivalent conditions of Lemma 4.4. The full subcategory of finitely generated $I$-contramodule $R$-modules is closed under the passages to subobjects, quotient objects, and extensions in $R-\bmod _{I \text {-ctra }}$ (and under the passages to the kernels, cokernels, and extensions in $R$-mod). The abelian category of finitely generated $I$-contramodule $R$-modules has enough projective objects, which are precisely the direct summands of finitely generated free $I$-contramodule $R$-modules (i. e., of the finite direct sums of copies of $\mathfrak{R}$ ).

Lemma 4.5. The restriction of the forgetful functor $\mathfrak{R}$-mod $\longrightarrow R$-mod provides an isomorphism between the abelian categories of finitely generated $\mathfrak{R}$-modules and finitely generated I-contramodule $R$-modules.

Proof. This lemma also holds for any ideal $I$ in a Noetherian ring $R$. First of all, by [23, Theorem B.1.1], the forgetful functor is an isomorphism between the categories of $\mathfrak{R} I$-contramodule $\mathfrak{R}$-modules and $I$-contramodule $R$-modules. Alternatively, notice that for any sets $X$ and $Y$ one has $\operatorname{Hom}_{\mathfrak{R}}(\mathfrak{R}[[X]], \mathfrak{R}[[Y]]) \simeq \mathfrak{R}[[Y]]^{X} \simeq$

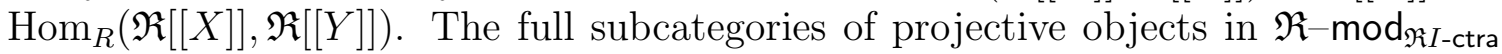


and $R-\bmod _{I \text {-ctra }}$ being equivalent and identified by the forgetful functor, it follows that the whole abelian categories are identified, too. Secondly, any finitely generated $\mathfrak{R}$-module is an $I$-contramodule, since $\mathfrak{R}$ is an $I$-contramodule and the class of $I$-contramodules is closed under cokernels and finite direct sums.

Now we return to the setting of a Notherian $\operatorname{ring} R$ with an ideal $I \subset R$ such that the quotient ring $R / I$ is Artinian. For any $R$-module $M$, we denote by $M^{\vee}$ the $R$-module $\operatorname{Hom}_{R}(M, C)$.

Proposition 4.6. (a) The restrictions of the functors $M \longmapsto M^{\vee}$ and $P \longmapsto P^{\vee}$ are mutually inverse anti-equivalences between the abelian categories of Artinian I-torsion $R$-modules and finitely generated I-contramodule $R$-modules.

(b) For any $I$-torsion $R$-module $M$ and any Artinian $I$-torsion $R$-module $L$, the functor $M \longmapsto M^{\vee}$ induces an isomorphism of the Hom modules

$$
\operatorname{Hom}_{R}(M, L) \simeq \operatorname{Hom}_{R}\left(L^{\vee}, M^{\vee}\right) .
$$

Proof. This is essentially the result of [13, Theorem 4.2]. One first notices that the functors $L \longmapsto L^{\vee}$ and $P \longmapsto P^{\vee}$ are mutually inverse anti-equivalences between the categories of injective Artinian $I$-torsion $R$-modules (i. e., direct summands of finite direct sums of copies of $C$ ) and projective finitely generated $I$-contramodule $R$-modules (i. e., direct summands of finite direct sums of copies of $\mathfrak{R}$ ). Representing arbitrary Artinian $I$-torsion $R$-modules as the kernels of morphisms between injectives and arbitrary finitely generated $I$-contramodule $R$-modules as the cokernels of morphisms between projectives, one can then deduce both the parts (a) and (b).

A finite complex of $R$-modules $N^{\bullet}$ is said to have $R$-flat dimension $\leqslant d$ if one has $H^{-n}\left(N^{\bullet} \otimes_{R}^{\mathbb{L}} M\right)=0$ for all $R$-modules $M$ and all the integers $n>d$. A finite complex of $R$-modules $N^{\bullet}$ is said to have $R$-projective dimension $\leqslant d$ if one has $H^{n} \mathbb{R} \operatorname{Hom}_{R}\left(N^{\bullet}, M\right)=0$ for all $R$-modules $M$ and all $n>d$.

A finite complex of $I$-torsion $R$-modules $L^{\bullet}$ is said to have $(R, I)$-contraflat $d i$ mension $\leqslant d$ if one has $H^{-n}\left(N^{\bullet} \otimes_{R}^{\mathbb{L}} P\right)=0$ for all $I$-contramodule $R$-modules $P$ and all the integers $n>d$. A finite complex of $I$-torsion $R$-modules $L^{\bullet}$ is said to have $(R, I)$-projective dimension $\leqslant d$ if one has $\operatorname{Hom}_{D^{\mathrm{b}}\left(R-\bmod _{I-\text { tors }}\right)}\left(L^{\bullet}, M[n]\right)=0$ for all $I$-torsion $R$-modules $M$ and all $n>d$.

We denote the $R$-flat dimension of a finite complex of $R$-modules $N^{\bullet}$ by $\operatorname{fd}_{R} N^{\bullet}$, the $R$-projective dimension of $N^{\bullet}$ by $\operatorname{pd}_{R} N^{\bullet}$, the $(R, I)$-contraflat dimension of a finite complex of $I$-torsion $R$-modules $L^{\bullet}$ by $\operatorname{cfd}_{(R, I)} L^{\bullet}$, and the $(R, I)$-projective dimension of $L^{\bullet}$ by $\operatorname{pd}_{(R, I)} L^{\bullet}$.

Proposition 4.7. For any finite complex of $I$-torsion $R$-modules $L^{\bullet}$, one has $\operatorname{pd}_{R} L^{\bullet} \geqslant \operatorname{pd}_{(R, I)} L^{\bullet} \geqslant \operatorname{cfd}_{(R, I)} L^{\bullet}=\operatorname{fd}_{R} L^{\bullet}=\operatorname{fd}_{\Re} L^{\bullet}$.

Proof. By the definition, one has $\operatorname{cfd}_{(R, I)} L \bullet \leqslant \operatorname{fd}_{R} L^{\bullet}$. Since $\mathfrak{R}$ is a flat $R$-module and all flat $\mathfrak{R}$-modules are also flat $R$-modules, for any finite complex of $\mathfrak{R}$-modules $N^{\bullet}$ one has $\operatorname{fd}_{R} N^{\bullet} \leqslant \operatorname{fd}_{\mathfrak{R}} N^{\bullet}$. Furthermore, a finite complex of $\mathfrak{R}$-modules $N^{\bullet}$ has $\mathfrak{R}$-flat dimension $\leqslant d$ whenever $H^{-n}\left(N^{\bullet} \otimes_{\mathfrak{R}}^{\mathbb{L}} M\right)=0$ for all finitely generated $\mathfrak{R}$-modules $M$ 
and all $n>d$. For a finite complex of $I$-torsion $R$-modules $L$ and an $\Re$-module $M$ one has $L^{\bullet} \otimes_{\mathfrak{R}} M=L^{\bullet} \otimes_{R} M$, hence $L^{\bullet} \otimes_{\mathfrak{R}}^{\mathbb{L}} M=L^{\bullet} \otimes_{R}^{\mathbb{L}} M$. Since finitely generated $\Re$-modules are $I$-contramodules, it follows that $\mathrm{fd}_{\mathfrak{R}} L^{\bullet} \leqslant \operatorname{cfd}_{(R, I)} L^{\bullet}$. We have shown that the three (contra)flat dimensions are equal to each other.

It remains to check the inequalities involving the projective dimensions. Applying Theorem 1.3 in the (easy) case $\star=\mathrm{b}$, one can see that $\operatorname{Hom}_{\mathrm{D}^{\mathrm{b}}\left(R-\bmod _{I \text {-tors }}\right)}\left(L^{\bullet}, M[n]\right)=$ $\operatorname{Hom}_{\mathrm{D}^{\mathrm{b}}(R-\mathrm{mod})}\left(L^{\bullet}, M[n]\right)$ for any finite complex of $I$-torsion $R$-modules $L^{\bullet}$ and any $I$-torsion $R$-module $M$. Hence $\operatorname{pd}_{R} L^{\bullet} \geqslant \operatorname{pd}_{(R, I)} L^{\bullet}$. Finally, for any $R$-module $M$ there is an isomorphism $\operatorname{Hom}_{R}\left(L \bullet \otimes_{R}^{\mathbb{L}} M, C\right) \simeq \mathbb{R} \operatorname{Hom}_{R}\left(L \bullet, \operatorname{Hom}_{R}(M, C)\right)$, and the $R$-module $\operatorname{Hom}_{R}(M, C)$ is $I$-torsion whenever an $R$-module $M$ is finitely generated. Besides, the $R$-modules $H^{-n}\left(L^{\bullet} \otimes_{R}^{\mathbb{L}} M\right)$ are $I$-torsion, so $H^{n} \operatorname{Hom}_{R}\left(L^{\bullet} \otimes_{R}^{\mathbb{L}} M, C\right)=0$ implies $H^{-n}\left(L^{\bullet} \otimes_{R}^{\mathbb{L}} M\right)=0$. This proves that $\operatorname{pd}_{(R, I)} L^{\bullet} \geqslant \operatorname{fd}_{R} L^{\bullet}$.

Now we come to the main definition of this section. As above, we assume that $R$ is a Noetherian commutative ring and $I \subset R$ is an ideal such that the quotient ring $R / I$ is Artinian. In this setting, a finite complex of $I$-torsion $R$-modules $B^{\bullet}$ is called a dedualizing complex for the ideal $I$ in the ring $R$ if

(i) the complex $B^{\bullet}$ has finite projective dimension as a complex of $I$-torsion $R$-modules, that is $\operatorname{pd}_{(R, I)} B^{\bullet}<\infty$;

(ii) the homothety map $\mathfrak{R} \longrightarrow \operatorname{Hom}_{\mathrm{D}^{\mathrm{b}}\left(R-\bmod _{I-\text { tors }}\right)}\left(B^{\bullet}, B^{\bullet}[*]\right)$ provided by the action of $\mathfrak{R}$ in $B^{\bullet}$ is an isomorphism of graded rings;

(iii) the cohomology modules of the complex $B^{\bullet}$ are Artinian $I$-torsion $R$-modules.

The dedualizing complex $B^{\bullet}$ is viewed as an object of the derived category $\mathrm{D}^{\mathrm{b}}\left(R-\bmod _{I \text {-tors }}\right)$. We refer to the book [8, Chapter 5], and additionally to the paper [27] and the references therein, for a discussion of the classical notions of a dualizing complex over a commutative ring or a pair of noncommutative ones, after which the above definition is largely modelled.

The following example provides a natural choice of a dedualizing complex for any ideal $I$ in a Noetherian commutative ring $R$ with an Artinian quotient ring $R / I$.

Example 4.8. Consider the derived functor of maximal $I$-torsion submodule $\mathbb{R} \Gamma_{I}: \mathrm{D}^{+}(R-\bmod ) \longrightarrow \mathrm{D}^{+}\left(R-\bmod _{I \text {-tors }}\right)$. According to Lemma $1.2(\mathrm{~b})$, this functor takes $\mathrm{D}^{\mathrm{b}}(R$-mod $)$ into $\mathrm{D}^{\mathrm{b}}\left(R-\bmod _{I \text {-tors }}\right)$. Set $B^{\bullet}=\mathbb{R} \Gamma_{I}(R)$. We claim that the complex $B^{\bullet}$ is a dedualizing complex for the ideal $I \subset R$.

According to Proposition 4.7, in order to check the projective dimension condition (i) it suffices to show that $\operatorname{pd}_{R} B^{\bullet}<\infty$. The latter is clear since, by Lemma 1.2(a), the complex $B^{\bullet}$ is isomorphic to the telescope complex $\operatorname{Tel}^{\bullet}(R, \mathbf{s})$ in the derived category $\mathrm{D}^{\mathrm{b}}(R$-mod) for any finite generating sequence $\mathbf{s}$ of the ideal $I \subset R$. In view of Lemma 2.5, the property (ii) is implicit in Theorem 3.4 in the case $\star=\mathrm{b}$ (see Example 5.8 below for further details).

Let us now prove the condition (iii). According to Lemma 4.2, it suffices to show that the complex ${ }_{n}^{\mathbb{R}} B^{\bullet}$ has finitely generated $R / I^{n}$-modules of cohomology. The functor $\Gamma_{I}$ takes injective objects in $R$-mod to injective objects in $R$ - $\bmod _{I \text {-tors }}$, so the 
composition of derived functors ${ }_{n}^{\mathbb{R}} \mathbb{R} \Gamma_{I}$ is the derived functor of the composition of left exact functors $M \longmapsto{ }_{n} \Gamma_{I}(M)$.

The latter functor assigns to an $R$-module $M$ its maximal $R / I^{n}$-submodule ${ }_{n} M$. It remains to notice that the functor $\operatorname{Ext}_{R}^{*}\left(R / I^{n}, M\right)$ takes finitely generated $R$-modules to cohomologically graded $R$-modules with finitely generated components, because it can be computed in terms of a left resolution of the $R$-module $R / I^{n}$ by finitely generated projective $R$-modules.

The following theorem is the main result of this section.

Theorem 4.9. Given a dedualizing complex $B^{\bullet}$ for an ideal $I$ in a Noetherian commutative ring $R$ with an Artinian quotient ring $R / I$, for any symbol $\star=\mathrm{b},+,-, \varnothing$, abs + , abs-, or abs there is an equivalence of derived categories $\mathrm{D}^{\star}\left(R-\bmod _{I \text {-tors }}\right) \simeq$ $\mathrm{D}^{\star}\left(R-\bmod _{I \text {-ctra }}\right)$ provided by mutually inverse functors $\mathbb{R} \operatorname{Hom}_{R}\left(B^{\bullet},-\right)$ and $B^{\bullet} \otimes_{R}^{\mathbb{L}}-$.

Proof. Assume for simplicity of notation that the complex $B^{\bullet}$ is concentrated in nonpositive cohomological degrees. Let $d$ be an integer greater or equal to the projective dimension $\operatorname{pd}_{(R, I)} B^{\bullet}$. By Proposition 4.7, we then also have $d \geqslant \operatorname{cfd}_{(R, I)} B^{\bullet}$.

To construct the image of a complex of $I$-torsion $R$-modules $M^{\bullet}$ under the functor $\mathbb{R} \operatorname{Hom}_{R}\left(B^{\bullet},-\right)$, one has to choose an exact sequence of complexes of $I$-torsion $R$-modules $0 \longrightarrow M^{\bullet} \longrightarrow J^{0} \bullet \longrightarrow J^{1, \bullet} \longrightarrow \cdots$ with injective $I$-torsion $R$-modules $J^{j, i}$. Then one applies the functor $\operatorname{Hom}_{R}\left(B^{\bullet},-\right)$ to every complex $0 \longrightarrow J^{0, i} \longrightarrow J^{1, i} \longrightarrow J^{2, i} \longrightarrow \cdots$, obtaining a nonnegatively graded complex of $I$-contramodule $R$-modules $0 \longrightarrow P^{0, i} \longrightarrow P^{1, i} \longrightarrow P^{2, i} \longrightarrow \cdots$. According to the projective dimension condition on the dedualizing complex $B^{\bullet}$, the complex $P^{\bullet}, i$ has zero cohomology modules at the cohomological degrees above $d$; so it is quasi-isomorphic to its canonical truncation complex $\tau_{\leqslant d} P^{\bullet}, i$. By the definition, one sets the object $\mathbb{R} \operatorname{Hom}_{R}\left(B^{\bullet}, M^{\bullet}\right)$ in the derived category $\mathrm{D}^{\star}\left(R\right.$ - $\left.\bmod _{I \text {-ctra }}\right)$ to be represented by the total complex of the bicomplex $\tau_{\leqslant d} P^{\bullet}, \bullet$ concentrated in the cohomological degrees $0 \leqslant j \leqslant d$ and $i \in \mathbb{Z}$.

Similarly, to construct the image of a complex of $I$-contramodule $R$-modules $P^{\bullet}$ under the functor $B^{\bullet} \otimes_{R}^{\mathbb{L}}-$, one has to choose an exact sequence of complexes of $I$-contramodule $R$-modules $\cdots \longrightarrow F^{-1, \bullet} \longrightarrow F^{0, \bullet} \longrightarrow P^{\bullet} \longrightarrow 0$ with projective $I$-contramodule $R$-modules $F^{j, i}$. Then one applies the functor $B^{\bullet} \otimes_{R}-$ to every complex $\cdots \longrightarrow F^{-2, i} \longrightarrow F^{-1, i} \longrightarrow F^{0, i} \longrightarrow 0$, obtaining a nonpositively graded complex of $I$-torsion $R$-modules $\cdots \longrightarrow M^{-2, i} \longrightarrow M^{-1, i} \longrightarrow M^{0, i} \longrightarrow 0$. By [33, Theorem 3.4] or [23, Proposition B.9.1], the projective $I$-contramodule $R$-modules are flat $R$-modules. According to the contraflat dimension condition on the complex $B^{\bullet}$, the complex $M^{\bullet}, i$ has zero cohomology modules at the cohomological degrees below $-d$; so it is quasi-isomorphic to its canonical truncation complex $\tau_{\geqslant-d} M^{\bullet, i}$. One sets the object $B^{\bullet} \otimes_{R}^{\mathbb{L}} P^{\bullet}$ in the derived category $\mathrm{D}^{\star}\left(R-\bmod _{I \text {-tors }}\right)$ to be represented by the total complex of the bicomplex $\tau_{\geqslant-d}\left(M^{\bullet}, \bullet\right)$ concentrated in the cohomological degrees $-d \leqslant j \leqslant 0$ and $i \in \mathbb{Z}$.

These constructions of two derived functors are but particular cases of the construction of a derived functor of finite homological dimension spelled out in Appendix B. 
According to the results of that appendix, the above constructions produce welldefined triangulated functors $\mathbb{R} \operatorname{Hom}_{R}\left(B^{\bullet},-\right): \mathrm{D}^{\star}\left(R-\bmod _{I \text {-tors }}\right) \longrightarrow \mathrm{D}^{\star}\left(R-\bmod _{I \text {-ctra }}\right)$ and $B^{\bullet} \otimes_{R}^{\mathbb{L}}-: \mathrm{D}^{\star}\left(R-\bmod _{I \text {-ctra }}\right) \longrightarrow \mathrm{D}^{\star}\left(R-\bmod _{I \text {-tors }}\right)$ for any derived category symbol $\star=\mathrm{b},+,-, \varnothing$, abs + , abs-, or abs. Moreover, the former functor is right adjoint to the latter one. All these assertions only depend on the first condition (i) in the definition of a dedualizing complex.

It remains to prove that the adjunction morphisms are isomorphisms. Since the total complexes of finite acyclic complexes of complexes are absolutely acyclic, in order to check that the morphism $P^{\bullet} \longrightarrow \mathbb{R} \operatorname{Hom}_{R}\left(B^{\bullet}, B^{\bullet} \otimes_{R}^{\mathbb{L}} P^{\bullet}\right)$ is an isomorphism in the derived category $\mathrm{D}^{\star}\left(R-\bmod _{I \text {-ctra }}\right)$ for all the $\star$-bounded complexes of $I$-contramodule $R$-modules $P^{\bullet}$ it suffices to consider the case of a one-term complex $P^{\bullet}=P$ corresponding to a single $I$-contramodule $R$-module $P$. Furthermore, since a morphism in $\mathrm{D}^{\mathrm{b}}\left(R-\bmod _{I \text {-ctra }}\right)$ is an isomorphism whenever it is an isomorphism in $\mathrm{D}^{-}\left(R-\bmod _{I \text {-ctra }}\right)$, one can view the one-term complex $P$ as an object of the bounded above derived category $\mathrm{D}^{-}\left(R-\bmod _{I \text {-ctra }}\right)$ and replace it with a free $I$-contramodule $R$-module resolution $F^{\bullet}$ of the contramodule $P$. Applying the same totalization argument to the complex $F^{\bullet}$, the question reduces to proving that the adjunction morphism $F \longrightarrow \mathbb{R} \operatorname{Hom}_{R}\left(B^{\bullet}, B^{\bullet} \otimes_{R}^{\mathbb{L}} F\right)$ is an isomorphism in $\mathrm{D}^{\mathrm{b}}\left(R-\bmod _{I \text {-ctra }}\right)$ for any free $I$-contramodule $R$-module $F$.

So let $X$ be a set and $F=\mathfrak{R}[[X]]$ be the $I$-contramodule $R$-module generated by $X$; then one has $B^{\bullet} \otimes_{R}^{\mathbb{L}} F=B^{\bullet} \otimes_{R} F=B^{\bullet}[X]$. According to the condition (iii) together with Lemma 4.3, there exists a bounded below complex of finitely cogenerated cofree $I$-torsion $R$-modules $E^{\bullet}$ together with a quasi-isomorphism of complexes of $I$-torsion $R$-modules $B^{\bullet} \longrightarrow E^{\bullet}$. We have to check that the natural map

$$
\mathfrak{R}[[X]] \longrightarrow \operatorname{Hom}_{R}\left(B^{\bullet}, E^{\bullet}[X]\right)
$$

is a quasi-isomorphism of complexes of $I$-contramodule $R$-modules.

Consider the morphism of complexes of $I$-torsion $R$-modules

$$
B^{\bullet} \otimes_{R} \operatorname{Hom}_{R}\left(E^{\bullet}, C\right) \longrightarrow C
$$

induced by the morphism $B^{\bullet} \longrightarrow E^{\bullet}$. Applying the functor $M \longmapsto M^{\vee}=$ $\operatorname{Hom}_{R}(M, C)$ to the morphism (5) and taking into account Proposition 4.6(b), we get the map

$$
\mathfrak{R}=\operatorname{Hom}_{R}(C, C) \longrightarrow \operatorname{Hom}_{R}\left(E^{\bullet \vee}, B^{\bullet \vee}\right)=\operatorname{Hom}_{R}\left(B^{\bullet}, E^{\bullet}\right),
$$

which is a quasi-isomorphism of complexes of ( $I$-contramodule) $R$-modules by the condition (ii). It follows that the morphism (5) is a quasi-isomorphism of complexes of $I$-torsion $R$-modules.

Now, applying the functor $M \longmapsto \operatorname{Hom}_{R}(M, C[X])$ to the quasi-isomorphism (5) and noticing that $\operatorname{Hom}_{R}\left(E^{\bullet}, C\right)=E^{\bullet \vee}$ is a complex of finitely generated (free) $\mathfrak{R}$-modules and $\operatorname{Hom}_{R}\left(E^{\bullet \vee}, C\right)=E^{\bullet}$ by Proposition 4.6(a), we obtain the desired 
quasi-isomorphism of complexes of $I$-contramodule $R$-modules

$$
\begin{aligned}
\mathfrak{R}[[X]]=\operatorname{Hom}_{R}(C, C[X]) & \longrightarrow \operatorname{Hom}_{R}\left(B^{\bullet}, \operatorname{Hom}_{R}\left(E^{\bullet \vee}, C[X]\right)\right) \\
& =\operatorname{Hom}_{R}\left(B^{\bullet}, \operatorname{Hom}_{R}\left(E^{\bullet \vee}, C\right)[X]\right)=\operatorname{Hom}_{R}\left(B^{\bullet}, E^{\bullet}[X]\right) .
\end{aligned}
$$

Similarly, in order to prove that the adjunction morphism $B^{\bullet} \otimes_{R}^{\mathbb{L}} \mathbb{R} \operatorname{Hom}_{R}\left(B^{\bullet}, M^{\bullet}\right)$ $\longrightarrow M^{\bullet}$ is an isomorphism in the derived category $\mathrm{D}^{\star}\left(R-\bmod _{I \text {-tors }}\right)$ for any $\star$-bounded complex of $I$-torsion $R$-modules $M^{\bullet}$, it suffices to check that this morphism is an isomorphism in $\mathrm{D}^{\mathrm{b}}\left(R-\bmod _{I \text {-tors }}\right)$ for any cofree $I$-torsion $R$-module viewed as a oneterm complex in $\mathrm{D}^{\mathrm{b}}\left(R-\bmod _{I \text {-tors }}\right)$. Let $J=C[X]$ be a cofree $I$-torsion $R$-module; then one has $\mathbb{R} \operatorname{Hom}_{R}\left(B^{\bullet}, J\right)=\operatorname{Hom}_{R}\left(B^{\bullet}, J\right)=\operatorname{Hom}_{R}\left(B^{\bullet}, C[X]\right)$.

As above, let $E^{\bullet}$ be a bounded below complex of finitely cogenerated cofree $I$-torsion $R$-modules endowed with a quasi-isomorphism $B^{\bullet} \longrightarrow E^{\bullet}$. Then $\operatorname{Hom}_{R}\left(E^{\bullet}, C\right)$ is a bounded above complex of (finitely generated) free $I$-contramodule $R$-modules quasi-isomorphic to $\operatorname{Hom}_{k}\left(B^{\bullet}, C\right)$. We have to show that the map

$$
B^{\bullet} \otimes_{R} \operatorname{Hom}_{R}\left(E^{\bullet}, C[X]\right) \longrightarrow C[X]
$$

is a quasi-isomorphism (of complexes of $I$-torsion $R$-modules).

Now, the natural map into the left-hand side from the $X$-indexed direct sum of copies of the complex $B^{\bullet} \otimes_{R} \operatorname{Hom}_{R}\left(E^{\bullet}, C\right)$ is an isomorphism, because $\operatorname{Hom}_{R}(C, C[X])=\mathfrak{R}[[X]]$ and $B^{\bullet} \otimes_{R} \mathfrak{R}[[X]]=B^{\bullet}[X]$. It remains to recall the quasi-isomorphism (5).

Remark 4.10. Returning to the discussion of the dualizing and dedualizing complexes in the introduction - the definition of a dualizing complex for a Noetherian commutative ring $R$ with an ideal $I \subset R$ was given in [24, Section C.1] (a somewhat more general definition of a dualizing complex for a Noetherian formal scheme can be found in the much earlier [32, Section 5]). In particular, when the quotient $\operatorname{ring} R / I$ is Artinian, the injective $R$-module $C$, viewed as a one-term complex, is a dualizing complex for $(R, I)$.

Note that this is not the same thing as a dualizing complex for the ring $R$, even in the case of a maximal ideal in a complete local ring. Rather, for any Noetherian commutative ring $R$ with an ideal $I \subset R$, if $D_{R}^{\bullet}$ is a dualizing complex for $R$, then $D^{\bullet}=\mathbb{R} \Gamma_{I}\left(D_{R}^{\bullet}\right)$ is a dualizing complex for $(R, I)$. E. g., consider the case of the ring of formal power series $R=k[[z]]$ in one variable $z$ over a field $k$. Then the ring $R$, being regular, can be considered as a dualizing complex over itself. Replacing it with a quasi-isomorphic complex of injective $R$-modules, we obtain a two-term complex $k((z)) \longrightarrow k((z)) / k[[z]]$, where $k((z))$ is the field of Laurent power series. To obtain a dualizing complex for the ideal $I=z k[[z]] \subset R$, one drops the uniquely divisible component $k((z))$, arriving to the one-term complex of $R$-modules $k((z)) / k[[z]] \simeq C$ [32, Example 5.1].

For any Noetherian commutative ring $R$ with an ideal $I$ such that the quotient ring $R / I$ is Artinian, the functors $\operatorname{Hom}_{R}(C,-)$ and $C \otimes_{R}-$, taking $C[X]$ to $\mathfrak{R}[[X]]$ and back, establish a covariant equivalence between the additive categories of injective $I$-torsion $R$-modules and projective $I$-contramodule $R$-modules. The homotopy 
category of unbounded complexes of injective objects in $R$ - $\bmod _{I \text {-tors }}$ is equivalent to the coderived category $\mathrm{D}^{\mathrm{co}}\left(R-\bmod _{I \text {-tors }}\right)$, while the homotopy category of unbounded complexes of projective objects in $R$ - $\bmod _{I \text {-ctra }}$ is equivalent to the contraderived category $\mathrm{D}^{\mathrm{ctr}}\left(R-\bmod _{I \text {-ctra }}\right)$. Therefore [24, Theorem C.1.4], the derived functors of Hom and tensor product with the dualizing complex $D^{\bullet}=C$ for $(R, I)$ provide an equivalence between the coderived and the contraderived categories

$$
\mathrm{D}^{\mathrm{co}}\left(R-\bmod _{I \text {-tors }}\right) \simeq \mathrm{D}^{\mathrm{ctr}}\left(R-\bmod _{I \text {-ctra }}\right) .
$$

On the other hand, according to our Theorem 4.9, the derived functors of Hom and tensor product with the dedualizing complex $B^{\bullet}$ (e. g., $B^{\bullet}=\mathbb{R} \Gamma_{I}(R)$, as in Example 4.8) provide equivalences of the conventional and absolute derived categories

$$
\mathrm{D}\left(R-\bmod _{I \text {-tors }}\right) \simeq \mathrm{D}\left(R-\bmod _{I \text {-ctra }}\right) \quad \text { and } \quad \mathrm{D}^{\text {abs }}\left(R-\bmod _{I \text {-tors }}\right) \simeq \mathrm{D}^{\text {abs }}\left(R-\bmod _{I \text {-ctra }}\right) \text {. }
$$

For an ideal $I$ in a regular Noetherian ring $R$ (with an Artinian quotient ring $R / I$ ), one can choose $B^{\bullet}=D^{\bullet}$. In this case, the abelian categories $R-\bmod _{I \text {-tors }}$ and $R$ - $\bmod _{I \text {-ctra }}$ have finite homological dimension, so there is no difference between the conventional and exotic derived category constructions for them.

\section{Dedualizing Complexes for Weakly Proregular Ideals}

The aim of this section is to prove Theorem 5.10. Taken together with Example 5.8, it extends the MGM duality result established in Corollary 3.5 to the absolute derived categories $\mathrm{D}^{\mathrm{abs}+}, \mathrm{D}^{\mathrm{abs}-}$, and $\mathrm{D}^{\mathrm{abs}}$ of torsion modules and contramodules over a commutative ring $R$ with a weakly proregular ideal $I$.

Let $I$ be a finitely generated ideal in a commutative ring $R$, and let $s_{1}, \ldots, s_{m}$ be a sequence of its generators, denoted by $\mathbf{s}$ for brevity. For any element $s \in R$, the complex $T^{\bullet}(R, s)$ from Section 2 is the union of its subcomplexes of free $R$-modules $T_{n}^{\bullet}(R, s)$ generated by the symbols $\epsilon_{0}, \ldots, \epsilon_{n-1}$ in degree 0 and $\delta_{1}, \ldots, \delta_{n}$ in degree 1. Accordingly, the complex $T^{\bullet}(R, \mathbf{s})$ is the union of its subcomplexes of finitely generated free $R$-modules $T_{n}^{\bullet}\left(R, s_{1}\right) \otimes_{R} \otimes \cdots \otimes_{R} T_{n}^{\bullet}\left(R, s_{m}\right)$. The complex $T_{n}^{\bullet}(R, \mathbf{s})$ is homotopy equivalent to the complex $\operatorname{Tel}_{n}^{\bullet}(R, \mathbf{s})$.

Let us denote the dual complexes by $K_{\bullet}^{n}(R, \mathbf{s})=\operatorname{Hom}_{R}\left(T_{n}^{\bullet}(R, \mathbf{s}), R\right)$. In particular, $K_{\bullet}^{1}(R, \mathbf{s})$ is the conventional Koszul complex associated with the sequence of elements $s_{1}, \ldots, s_{m} \in R$, while the complex $K_{\bullet}^{n}(R, \mathbf{s})$ is quasi-isomorphic to the Koszul complex $K_{\bullet}^{1}\left(R, \mathbf{s}^{n}\right)$ associated with the sequence of elements $s_{1}^{n}, \ldots, s_{m}^{n} \in R$.

The Thomason-Trobaugh-Neeman theory of compactly generated triangulated categories is fairly widely known by now (see, e. g., [16] or [29]). The following one is an old result.

Proposition 5.1. For any given $n \geqslant 1$, the complex $K_{\bullet}^{n}(R, \mathbf{s})$ is a compact generator of the triangulated subcategory $\mathrm{D}_{\text {I-tors }}(R$-mod $) \subset \mathrm{D}(R$-mod $)$ of complexes with $I$-torsion cohomology modules in the derived category of $R$-modules. A complex of $R$-modules with $I$-torsion cohomology modules is a compact object of $\mathrm{D}_{I \text {-tors }}(R$-mod $)$ if and only if it is a compact object of $\mathrm{D}(R-\mathrm{mod})$. 
Proof. The first assertion goes back to [2, Proposition 6.1]; see also [29, Proposition 6.6]. It suffices to consider the case $n=1$. One can easily see that the complexes $K_{\bullet}^{n}(R, \mathbf{s})$, viewed as objects of the homotopy category $\operatorname{Hot}(R-$ mod $)$, can be obtained from the complex $K_{\bullet}^{1}(R, \mathbf{s})$ using the operations of shift and cone. Let $M^{\bullet}$ be a complex of $R$-modules with $I$-torsion cohomology modules. Then the inductive limit of the complexes $\operatorname{Hom}_{R}\left(K_{\bullet}^{n}(R, \mathbf{s}), M^{\bullet}\right) \simeq T_{n}^{\bullet}(R, \mathbf{s}) \otimes_{R} M^{\bullet}$ is quasi-isomorphic to the complex $M^{\bullet}$ by Lemma 1.1(c). Hence the complex $M^{\bullet}$ is acyclic whenever all the complexes $\operatorname{Hom}_{R}\left(K_{\bullet}^{n}(R, \mathbf{s}), M^{\bullet}\right)$ are. The complexes $K_{\bullet}^{n}(R, \mathbf{s})$ are compact objects in $\mathrm{D}_{I \text {-tors }}(R$-mod $)$, since they are compact in $\mathrm{D}(R-$ mod $)$. This proves the first assertion. It follows that the compact objects of $\mathrm{D}_{I \text {-tors }}(R-$ mod $)$ are precisely those objects that can be obtained from $K_{\bullet}^{1}(R, \mathbf{s})$ using the operations of shift, cone, and the passage to a direct summand, implying the second one (cf. [19, Lemma 5.3]).

Lemma 5.2. (a) For any $I$-torsion $R$-module $M$, the natural morphism of complexes of $R$-modules

$$
T^{\bullet}(R, \mathbf{s}) \otimes_{R} \operatorname{Hom}_{R}\left(T^{\bullet}(R, \mathbf{s}), M\right) \longrightarrow M
$$

is a quasi-isomorphism.

(b) For any $I$-contramodule $R$-module $P$, the natural morphism of complexes of $R$-modules

$$
P \longrightarrow \operatorname{Hom}_{R}\left(T^{\bullet}(R, \mathbf{s}), T^{\bullet}(R, \mathbf{s}) \otimes_{R} P\right)
$$

is a quasi-isomorphism.

Proof. These computations are implicit in the proof of Theorem 3.4. In view of Lemma 1.1(c), part (a) generalizes to the assertion that for any $R$-module $M$ the morphism

$$
T^{\bullet}(R, \mathbf{s}) \otimes_{R} M \longrightarrow T^{\bullet}(R, \mathbf{s}) \otimes_{R} \operatorname{Hom}_{R}\left(T^{\bullet}(R, \mathbf{s}), M\right)
$$

induced by the natural morphism of complexes $T^{\bullet}(R, \mathbf{s}) \longrightarrow R$ is a quasiisomorphism. This is essentially [18, Lemma 7.6]. One shows that the morphism of complexes

$$
T_{n}^{\bullet}(R, \mathbf{s}) \otimes_{R} M \longrightarrow \operatorname{Hom}_{R}\left(T^{\bullet}(R, \mathbf{s}), T_{n}^{\bullet}(R, \mathbf{s}) \otimes_{R} M\right)
$$

is a quasi-isomorphism for every $n \geqslant 1$. A cocone of the latter morphism is a complex computing the $R$-modules of morphisms $\operatorname{Hom}_{\mathrm{D}^{\mathrm{b}}(R-\text { mod })}\left(C_{\mathbf{s}}^{\bullet}(R), T_{n}^{\bullet}(R, \mathbf{s}) \otimes_{R}\right.$ $M[*])$ in the derived category $\mathrm{D}^{\mathrm{b}}(R$-mod). So it suffices to check that the $R$-modules $\operatorname{Hom}_{\mathrm{D}^{\mathrm{b}}(R-\mathrm{mod})}\left(C_{\mathbf{s}}^{i}(R), T_{n}^{\bullet}(R, \mathbf{s}) \otimes_{R} M[*]\right)$ vanish for every $i \geqslant 0$. Indeed, the $R$-module $C_{\mathbf{s}}^{i}(R)$ is a finite direct sum of $R$-modules in each of which one of the elements $s_{j}$ acts invertibly, while in the complex $T_{n}^{\bullet}(R, \mathbf{s}) \otimes_{R} M$ all the elements $s_{j}^{n}$ act contractibly.

Similarly, in view of Lemma 2.2(c), part (b) generalizes to the assertion that for any $R$-module $M$ the morphism

$$
\operatorname{Hom}_{R}\left(T^{\bullet}(R, \mathbf{s}), T^{\bullet}(R, \mathbf{s}) \otimes_{R} M\right) \longrightarrow \operatorname{Hom}_{R}\left(T^{\bullet}(R, \mathbf{s}), M\right)
$$

induced by the morphism of complexes $T^{\bullet}(R, \mathbf{s}) \longrightarrow R$ is a quasi-isomorphism. This is essentially [18, Lemma 7.2]. A cocone of the morphism $T^{\bullet}(R, \mathbf{s}) \otimes_{R} M \longrightarrow M$ is quasi-isomorphic to the complex $C_{\mathbf{s}}^{\bullet}(M)$, so it suffices to check that the complex $\operatorname{Hom}_{R}\left(T^{\bullet}(R, \mathbf{s}), C_{\mathbf{s}}^{\bullet}(M)\right)$ is acyclic. Indeed, let us show that the complex 
$\operatorname{Hom}_{R}\left(T^{\bullet}(R, \mathbf{s}), C_{\mathbf{s}}^{i}(M)\right)$ is acyclic for every $i \geqslant 0$. By Lemma 2.2(a), the cohomology modules of this complex are finite direct sums of $R$-modules each of which is simultaneously an $s_{j}$-contramodule and an $R\left[s_{j}^{-1}\right]$-module for one of the elements $s_{j}$. All such modules vanish.

Let $\mathfrak{R}=\lim _{n} R / I^{n}$ denote the $I$-adic completion of the ring $R$. The ring $\mathfrak{R}$ is a complete and separated topological ring in its projective limit topology, which coincides with its $I$-adic topology. An $I$-torsion $R$-module is the same thing as a discrete $\mathfrak{R}$-module. For any set $X$, we denote by $\mathfrak{R}[[X]]=\lim _{n} R / I^{n}[X]$ the $R$-module of all maps of sets $f: X \longrightarrow \mathfrak{R}$ converging to zero in the topology of $\mathfrak{R}$ (i. e., for any open subset $U \subset \mathfrak{R}$ one has $f(x) \in U$ for all but a finite number of elements $x \in X)$. As any $R$-module that is separated and complete in its $I$-adic topology, $\mathfrak{R}[[X]]$ is an $I$-contramodule (see Section 2 ).

For the rest of the section we assume that the ideal $I \subset R$ is weakly proregular. Then the $R$-module $\mathfrak{R}[[X]]$ is called the free $I$-contramodule $R$-module generated by the set $X$ (cf. [26, Section 2.1]). Part (a) of the next lemma justifies the terminology.

Lemma 5.3. (a) For any I-contramodule $R$-module $P$ there is a bijective correspondence between the $R$-module morphisms $\mathfrak{R}[[X]] \longrightarrow P$ and the maps of sets $X \longrightarrow P$. Free $I$-contramodule $R$-modules are projective objects in the abelian category $R$ - $\bmod _{I \text {-ctra, }}$, there are enough of them, and any projective $I$-contramodule $R$-module is a direct summand of a free one.

(b) For any set $X$ and any integer $n \geqslant 1$, the morphisms of complexes

$$
C_{\mathbf{s}}^{\bullet}(R[X])^{\sim} \longrightarrow C_{\mathbf{s}}^{\bullet}(\Re[[X]])^{\sim} \quad \text { and } \quad T_{n}^{\bullet}(R, \mathbf{s}) \otimes_{R} R[X] \longrightarrow T_{n}^{\bullet}(R, \mathbf{s}) \otimes_{R} \mathfrak{R}[[X]]
$$

induced by the completion map $R[X] \longrightarrow \mathfrak{R}[[X]]$ are quasi-isomorphisms.

(c) For any set $X$, the morphism of complexes

$$
\operatorname{Hom}_{R}\left(T^{\bullet}(R, \mathbf{s}), R[X]\right) \longrightarrow \operatorname{Hom}_{R}\left(T^{\bullet}(R, \mathbf{s}), \mathfrak{R}[[X]]\right)
$$

induced by the completion map $R[X] \longrightarrow \mathfrak{R}[[X]]$ is a quasi-isomorphism.

Proof. Part (a): by Lemma 2.5, one has $\Delta_{I}(R[X]) \simeq \mathfrak{R}[[X]]$, so $\operatorname{Hom}_{R}(R[X], P) \simeq$ $\operatorname{Hom}_{R}(\mathfrak{R}[[X]], P)$ for any $I$-contramodule $P$. The remaining assertions immediately follow. Parts (b-c): by Lemmas 2.5 and 2.7(a), the complex $\operatorname{Hom}_{R}\left(T^{\bullet}(R, \mathbf{s}), R[X]\right)$ is quasi-isomorphic to the $R$-module $\mathfrak{R}[[X]]$ and the morphism of complexes $R[X] \longrightarrow$ $\operatorname{Hom}_{R}\left(T^{\bullet}(R, \mathbf{s}), R[X]\right)$ computes the completion morphism $R[X] \longrightarrow \mathfrak{R}[[X]]$. Now part (b) is provided by the proof of Lemma 5.2(a) and part (c) follows from Lemma 3.2(a).

It was shown in [33, Theorem 3.4] and [23, Proposition B.9.1] (see also [20, Theorem 1.5(2)] and [24, Proposition C.5.4]) that the free $I$-contramodule $R$-modules $\mathfrak{R}[[X]]$ are flat $R$-modules when the ring $R$ is Noetherian. Part (c) of the following lemma is a weak generalization of this result to the weakly proregular case.

Lemma 5.4. (a) For any finite complex of finitely generated projective $R$-modules $K^{\bullet}$ with $I$-torsion cohomology modules, the complex $\operatorname{Hom}_{R}\left(K^{\bullet}, R\right)$ also has I-torsion 
cohomology modules. Moreover, for any complex of $R$-modules $M^{\bullet}$, the complex $\operatorname{Hom}_{R}\left(K^{\bullet}, M^{\bullet}\right)$ has I-torsion cohomology modules.

(b) For any finite complex of finitely generated projective $R$-modules $K^{\bullet}$ with $I$-torsion cohomology modules and any set $X$, the morphism of complexes of $R$-modules

$$
K^{\bullet} \otimes_{R} R[X] \longrightarrow K^{\bullet} \otimes_{R} \Re[[X]]
$$

induced by the completion map $R[X] \longrightarrow \mathfrak{R}[[X]]$ is a quasi-isomorphism.

(c) Let $K^{\bullet} \longrightarrow M^{\bullet}$ be a quasi-isomorphism between a complex of I-torsion $R$-modules $M^{\bullet}$ and a finite complex of finitely generated projective $R$-modules $K^{\bullet}$ with I-torsion cohomology modules. Then for any set $X$ the induced morphism

$$
K^{\bullet} \otimes_{R} \mathfrak{R}[[X]] \longrightarrow M^{\bullet} \otimes_{R} \mathfrak{R}[[X]]
$$

is a quasi-isomorphism of complexes of $R$-modules.

Proof. Part (a) does not depend on the weak proregularity assumption yet. To prove it, choose a finite sequence of elements $\mathbf{s}$ generating the ideal $I \subset R$ and an integer $n \geqslant 1$. By Proposition 5.1, the complex $K^{\bullet}$, viewed as an object of the homotopy category $\operatorname{Hot}(R$-mod), is a direct summand of a complex obtained from the complex $K_{\bullet}^{n}(R, \mathbf{s})$ using the operations of shift and cone. Since the complex $K_{\bullet}^{n}(R, \mathbf{s})$ is selfdual up to a shift, the first assertion follows. Moreover, all the cohomology modules of the complex $K_{\bullet}^{n}(R, \mathbf{s})$ are annihilated by a large enough power of the ideal $I$, and consequently so are all the cohomology modules of the complex $K^{\bullet}$ and every cohomology module of the complex $\operatorname{Hom}_{R}\left(K^{\bullet}, M^{\bullet}\right)$.

By virtue of the same argument based on Proposition 5.1, part (b) follows from Lemma 5.3(b). To prove part (c), notice that the morphism $K^{\bullet} \otimes_{R} R[X] \longrightarrow M^{\bullet} \otimes_{R}$ $R[X]$ is a quasi-isomorphism because the $R$-module $R[X]$ is flat, the morphism $M^{\bullet} \otimes_{R}$ $R[X] \longrightarrow M^{\bullet} \otimes_{R} \mathfrak{R}[[X]]$ is an isomorphism of complexes of $R$-modules since $M^{\bullet}$ is a complex of $I$-torsion $R$-modules, and the morphism $K^{\bullet} \otimes_{R} R[X] \longrightarrow K^{\bullet} \otimes_{R} \mathfrak{R}[[X]]$ is a quasi-isomorphism by part (b).

Clearly, the abelian category of $I$-torsion $R$-modules $R$-mod $\operatorname{m}_{I \text {-tors }}$ has enough injective objects. Moreover, the maximal $I$-torsion submodule $\Gamma_{I}(J)$ of any injective $R$-module $J$ is an injective object of $R-\bmod _{I \text {-tors }}$. There are enough injective objects of this particular form in $R$ - $\bmod _{I \text {-tors }}$, so any injective object of $R$ - $\bmod _{I \text {-tors }}$ a direct summand of the $R$-module $\Gamma_{I}(J)$ for some injective $R$-module $J$.

For a Noetherian ring $R$, it follows from the Artin-Rees lemma that any injective object of the category $R-\bmod _{I \text {-tors }}$ is simultaneously an injective object of the category $R$-mod. Part (b) of the next lemma can be sometimes used in lieu of this assertion in the case of a weakly proregular ideal $I$.

Lemma 5.5. (a) Let $J$ be an injective $R$-module and $H=\Gamma_{I}(J)$ be its maximal $I$-torsion submodule. Then for any bounded above complex of projective $R$-modules $L \bullet$ with I-torsion cohomology modules the morphism of complexes of $R$-modules

$$
\operatorname{Hom}_{R}\left(L^{\bullet}, H\right) \longrightarrow \operatorname{Hom}_{R}\left(L^{\bullet}, J\right)
$$

induced by the embedding map $H \longrightarrow J$ is a quasi-isomorphism. 
(b) Let $L^{\bullet} \longrightarrow M^{\bullet}$ be a quasi-isomorphism between a complex of I-torsion $R$-modules $M^{\bullet}$ and a bounded above complex of projective $R$-modules $L^{\bullet}$ with $I$-torsion cohomology modules. Then for any injective object $H$ of the abelian category of $I$-torsion $R$-modules the induced morphism

$$
\operatorname{Hom}_{R}\left(M^{\bullet}, H\right) \longrightarrow \operatorname{Hom}_{R}\left(L^{\bullet}, H\right)
$$

is a quasi-isomorphism of complexes of $R$-modules.

Proof. Part (a) is implicit in the adjunction of derived functors from the proof of Theorem 1.3 together with the result of Corollary 1.4. To give a direct proof, notice that by the weak proregularity assumption on the ideal $I$ the complex $C_{\mathbf{s}}^{\bullet}(J)^{\sim}$ is quasi-isomorphic to the $R$-module $H$ and the morphism of complexes $C_{\mathbf{s}}^{\bullet}(J)^{\sim} \longrightarrow J$ computes the embedding morphism $H \longrightarrow J$. The quasiisomorphism of complexes of $R$-modules $H \longrightarrow C_{\mathbf{s}}^{\bullet}(J)^{\sim}$ induces a quasi-isomorphism $\operatorname{Hom}_{R}\left(L^{\bullet}, H\right) \longrightarrow \operatorname{Hom}_{R}\left(L^{\bullet}, C_{\mathbf{s}}^{\bullet}(J)^{\sim}\right)$, so it remains to show that the complex $\operatorname{Hom}_{R}\left(L^{\bullet}, C_{\mathbf{s}}^{\bullet}(J)\right)$ is acyclic. Here one argues as in the proof of Lemma 5.2(b) together with the proof of Lemma 2.2(a). For every $i \geqslant 0$, the cohomology modules of the complex $\operatorname{Hom}_{R}\left(L^{\bullet}, C_{\mathbf{s}}^{i}(J)\right)$ are $I$-contramodule $R$-modules, since the complex $L \bullet \otimes_{R} T^{\bullet}(R, s)^{\prime}$ is contractible for any $s \in I$ as a bounded above complex of projective $R$-modules quasi-isomorphic to the acyclic complex $L^{\bullet}\left[s^{-1}\right]$. Being at the same time isomorphic to finite direct sums of $R$-modules each of which is an $R\left[s_{j}^{-1}\right]$-module for one of the elements $s_{j}$, these cohomology modules have to vanish.

Part (b) is deduced in the way similar to the proof of Lemma 5.4(c). One can assume that $H=\Gamma_{I}(J)$ for some injective $R$-module $J$, as there are enough injective $I$-torsion $R$-modules of this form. Then the morphism $\operatorname{Hom}_{R}\left(M^{\bullet}, J\right) \longrightarrow$ $\operatorname{Hom}_{R}\left(L^{\bullet}, J\right)$ is a quasi-isomorphism because the $R$-module $J$ is injective, the morphism $\operatorname{Hom}_{R}\left(M^{\bullet}, H\right) \longrightarrow \operatorname{Hom}_{R}\left(M^{\bullet}, J\right)$ is an isomorphism, and the morphism $\operatorname{Hom}_{R}\left(L^{\bullet}, H\right) \longrightarrow \operatorname{Hom}_{R}\left(L^{\bullet}, J\right)$ is a quasi-isomorphism by part (a).

Remark 5.6. In other words, Lemma 5.5(b) simply means that the Ext modules $\operatorname{Ext}_{R}^{i}(M, H)$ computed in the abelian category of $R$-modules between an $I$-torsion $R$-module $M$ and an injective $I$-torsion $R$-module $H$ vanish for all $i>0$. This is but a particular case of Theorem 1.3. Similarly, it follows from Theorem 2.9 that $\operatorname{Ext}_{R}^{i}(\mathfrak{R}[[X]], P)=0$ for any $I$-contramodule $R$-module $P$ and all $i>0$. Taking into account the natural isomorphism $\operatorname{Hom}_{R}\left(\operatorname{Tor}_{i}^{R}(M, N), J\right) \simeq \operatorname{Ext}_{R}^{i}\left(N, \operatorname{Hom}_{R}(M, J)\right)$ for an injective $R$-module $J$ and any $R$-modules $M, N$, together with the fact that the $R$-module $\operatorname{Hom}_{R}(M, J)$ is an $I$-contramodule for any $I$-torsion $R$-module $M$ and any $R$-module $J$ (see Section 2), one concludes that $\operatorname{Tor}_{i}^{R}(M, \mathfrak{R}[[X]])=0$ for any $I$-torsion $R$-module $M$ and any set $X$. This is a stronger version of Lemma 5.4(c).

As in Section 4, a finite complex of $I$-torsion $R$-modules $L^{\bullet}$ is said to have projective dimension $\leqslant d$ if one has $\operatorname{Hom}_{\mathrm{D}^{\mathrm{b}}\left(R \text {-mod } \operatorname{m}_{\text {-tors }}\right)}\left(L^{\bullet}, M[n]\right)=0$ for all $I$-torsion $R$-modules $M$ and all the integers $n>d$.

By Lemma 5.3(a), the bounded above derived category $\mathrm{D}^{-}\left(R-\bmod _{I \text {-ctra }}\right)$ is equivalent to the homotopy category of bounded above complexes of projective $I$-contramodule $R$-modules. Given a complex of $I$-torsion $R$-modules $M^{\bullet}$ 
and a bounded above complex of $I$-contramodule $R$-modules $P^{\bullet}$, we denote by $\operatorname{Ctrtor}_{*}^{(R, I)}\left(M^{\bullet}, P^{\bullet}\right)$ the homology $R$-modules

$$
\operatorname{Ctrtor}_{n}^{(R, I)}\left(M^{\bullet}, P^{\bullet}\right)=H^{-n}\left(M^{\bullet} \otimes_{R} F^{\bullet}\right)
$$

of the tensor product of the complex of $I$-torsion $R$-modules $M^{\bullet}$ with a bounded above complex of projective $I$-contramodule $R$-modules $F^{\bullet}$ quasi-isomorphic to the complex $P^{\bullet}$. By Remark 5.6, the functors $\operatorname{Ctrtor}_{n}^{(R, I)}\left(M^{\bullet}, P^{\bullet}\right)$ and $H^{-n}\left(M^{\bullet} \otimes_{R}^{\mathbb{L}} P^{\bullet}\right)$ agree wherever the former of them is defined.

A finite complex of $I$-torsion $R$-modules $L^{\bullet}$ is said to have contraflat dimension $\leqslant d$ if one has $\operatorname{Ctrtor}_{n}^{(R, I)}\left(L^{\bullet}, P\right)=0$ for all $I$-contramodule $R$-modules $P$ and all the integers $n>d$. The next one is the main definition of this section.

A finite complex of $I$-torsion $R$-modules $B^{\bullet}$ is called a dedualizing complex for the ideal $I \subset R$ if the following conditions hold:

(i) the complex $B^{\bullet}$ has finite projective and contraflat dimensions as a complex of $I$-torsion $R$-modules;

(ii) the homothety map $\mathfrak{R} \longrightarrow \operatorname{Hom}_{\mathrm{D}^{\mathrm{b}}\left(R-\bmod _{I \text {-tors }}\right)}\left(B^{\bullet}, B^{\bullet}[*]\right)$ is an isomorphism of graded rings;

(iii) for any finite complex of finitely generated projective $R$-modules $K^{\bullet}$ with $I$-torsion cohomology modules, the complex $\operatorname{Hom}_{R}\left(K^{\bullet}, B^{\bullet}\right)$ is a compact object of the derived category $\mathrm{D}\left(R-\bmod _{I \text {-tors }}\right)$.

The dedualizing complex $B^{\bullet}$ is viewed as an object of the derived category $\mathrm{D}^{\mathrm{b}}\left(R-\bmod _{I \text {-tors }}\right)$.

Theorem 5.7. Let I be a weakly proregular finitely generated ideal in a commutative ring $R$. Then for any finite complex of $I$-torsion $R$-modules $B^{\bullet}$ satisfying the conditions (ii-iii) there is an involutive anti-equivalence on the full subcategory of compact objects in $\mathrm{D}\left(R-\bmod _{I \text {-tors }}\right)$ provided by a derived functor $\mathbb{R} \operatorname{Hom}_{R}\left(-, B^{\bullet}\right)$.

Proof. By Theorem 1.3 and Corollary 1.4 , one has $\mathrm{D}\left(R-\bmod _{I \text {-tors }}\right)=\mathrm{D}_{I \text {-tors }}(R-$ mod $) \subset$ $\mathrm{D}(R-\mathrm{mod})$. According to Proposition 5.1, any compact object of $\mathrm{D}_{I \text {-tors }}(R-$ mod $)$ is also compact in $\mathrm{D}(R$-mod $)$, so the full subcategory of compact objects in $\mathrm{D}_{I \text {-tors }}(R$-mod) is equivalent to the homotopy category of finite complexes of finitely generated projective $R$-modules with $I$-torsion cohomology modules (see [1, Theorem 2.8]). To compute the image of a compact object $M^{\bullet} \in \mathrm{D}\left(R-\bmod _{\text {-tors }}\right)$ under the functor $\mathbb{R} \operatorname{Hom}_{R}\left(-, B^{\bullet}\right)$, one chooses a finite complex of finitely generated projective $R$-modules $K^{\bullet}$ together with a quasi-isomorphism of complexes of $R$-modules $K^{\bullet} \longrightarrow M^{\bullet}$ and applies the functor $\operatorname{Hom}_{R}\left(-, B^{\bullet}\right)$ to the complex $K^{\bullet}$. The natural isomorphism of complexes

$$
\operatorname{Hom}_{R}\left(L^{\bullet}, \operatorname{Hom}_{R}\left(K^{\bullet}, B^{\bullet}\right)\right) \simeq \operatorname{Hom}_{R}\left(K^{\bullet}, \operatorname{Hom}_{R}\left(L^{\bullet}, B^{\bullet}\right)\right)
$$

for any complexes of $R$-modules $K^{\bullet}$ and $L^{\bullet}$ makes the contravariant endofunctor $\mathbb{R} \operatorname{Hom}_{R}\left(-, B^{\bullet}\right)$ on the category of compact objects in $\mathrm{D}\left(R-\bmod _{I \text {-tors }}\right)$ right adjoint to itself. It remains to show that the adjunction morphism $K^{\bullet} \longrightarrow$ $\mathbb{R} \operatorname{Hom}_{R}\left(\operatorname{Hom}_{R}\left(K^{\bullet}, B^{\bullet}\right), B^{\bullet}\right)$ is an isomorphism in $\mathrm{D}\left(R-\bmod _{I \text {-tors }}\right)$ for any finite 
complex of finitely generated projective $R$-modules $K^{\bullet}$ with $I$-torsion cohomology modules.

Let ' $B^{\bullet}$ be a bounded below complex of injective $R$-modules endowed with a quasiisomorphism $B^{\bullet} \longrightarrow{ }^{\prime} B^{\bullet}$. Then it suffices to check that the natural morphism $K^{\bullet} \longrightarrow \operatorname{Hom}_{R}\left(\operatorname{Hom}_{R}\left(K^{\bullet}, B^{\bullet}\right),{ }^{\prime} B^{\bullet}\right) \simeq K^{\bullet} \otimes_{R} \operatorname{Hom}_{R}\left(B^{\bullet},{ }^{\prime} B^{\bullet}\right)$ is a quasi-isomorphism of complexes of $R$-modules. By the condition (ii) together with Theorem 1.3, the complex $\operatorname{Hom}_{R}\left(B^{\bullet},{ }^{\prime} B^{\bullet}\right)$ is quasi-isomorphic to the ring $\mathfrak{R}$, so we have to show that the morphism $K^{\bullet} \longrightarrow \mathfrak{R} \otimes_{R} K^{\bullet}$ is a quasi-isomorphism. But this is the particular case of Lemma 5.4(b) for a one-element set $X$.

The following example explains where one can get a dedualizing complex for an arbitrary weakly proregular finitely generated ideal in a commutative ring.

Example 5.8. Let $I$ be a weakly proregular finitely generated ideal in a commutative ring $R$. As in Example 4.8, consider the derived functor of maximal $I$-torsion submodule $\mathbb{R} \Gamma_{I}: \mathrm{D}^{\mathrm{b}}(R$-mod $) \longrightarrow \mathrm{D}^{\mathrm{b}}\left(R-\bmod _{I \text {-tors }}\right)$. It is claimed that the complex $B^{\bullet}=\mathbb{R} \Gamma_{I}(R)$ is a dedualizing complex for the ideal $I \subset R$.

Indeed, one has $\operatorname{Hom}_{\mathrm{D}^{\mathrm{b}}\left(R-\bmod _{I \text {-tors }}\right)}\left(B^{\bullet}, M[*]\right) \simeq \operatorname{Hom}_{\mathrm{D}^{\mathrm{b}}(R-\text { mod })}\left(B^{\bullet}, M[*]\right)$ for any $I$-torsion $R$-module $M$ by (the case $\star=\mathrm{b}$ of) Theorem 1.3, so the complex $B^{\bullet}$, having finite projective dimension as a complex of $R$-modules for the reason of being isomorphic to the complex $T^{\bullet}(R, \mathbf{s})$ in $\mathrm{D}^{\mathrm{b}}(R-\mathrm{mod})$, also has finite projective dimension as a complex of $I$-torsion $R$-modules.

Furthermore, the quasi-isomorphism of complexes of $R$-modules $T^{\bullet}(R, \mathbf{s}) \longrightarrow B^{\bullet}$ induces a quasi-isomorphism of tensor products $T^{\bullet}(R, \mathbf{s}) \otimes_{R} \mathfrak{R}[[X]] \longrightarrow B^{\bullet} \otimes_{R} \mathfrak{R}[[X]]$. Indeed, the complex $B^{\bullet}$ being a complex of $I$-torsion $R$-modules, the morphism $B^{\bullet} \otimes_{R}$ $R[X] \longrightarrow B^{\bullet} \otimes_{R} \mathfrak{R}[[X]]$ induced by the completion morphism $R[X] \longrightarrow \mathfrak{R}[[X]]$ is an isomorphism of complexes, while the morphism $T^{\bullet}(R, \mathbf{s}) \otimes_{R} R[X] \longrightarrow B^{\bullet} \otimes_{R}$ $R[X]$ is a quasi-isomorphism since the $R$-module $R[X]$ is flat, and the morphism $T^{\bullet}(R, \mathbf{s}) \otimes_{R} R[X] \longrightarrow T^{\bullet}(R, \mathbf{s}) \otimes_{R} \mathfrak{R}[[X]]$ is a quasi-isomorphism by Lemma 5.3(b). Since $T^{\bullet}(R, \mathbf{s})$ is a complex of flat $R$-modules, it follows that the homology $R$-modules of the complex $T^{\bullet}(R, \mathbf{s}) \otimes_{R} P$ are isomorphic to the $R$-modules $\operatorname{Ctrtor}_{*}^{(R, I)}\left(B^{\bullet}, P\right)$ for any $I$-contramodule $R$-module $P$, so the complex $B^{\bullet}$ has finite contraflat dimension, too (cf. Lemma 5.4(c) and Remark 5.6). This proves the condition (i).

The condition (ii) is provided by Lemma 5.2(b) applied to the $I$-contramodule $R$-module $\mathfrak{R}$ together with Lemma 5.3(b) applied to a one-element set $X$. Finally, by Proposition 5.1 it suffices to prove the condition (iii) for one of the complexes $K^{\bullet}=K_{\bullet}^{n}(R, \mathbf{s})$, for which it follows from Lemma 1.1(c), as the complex $\operatorname{Hom}_{R}\left(K_{\bullet}^{n}(R, \mathbf{s}), T^{\bullet}(R, \mathbf{s})\right)$ is quasi-isomorphic to $T_{n}^{\bullet}(R, \mathbf{s}) \otimes_{R} C_{\mathbf{s}}^{\bullet}(R)^{\sim}$ and to $T_{n}^{\bullet}(R, \mathbf{s})$.

According to Lemma 5.4(a), the involutive anti-equivalence $\operatorname{Hom}_{R}(-, R)$ of the category of finite complexes of finitely generated projective $R$-modules takes its full subcategory of complexes with $I$-torsion cohomology modules into itself. One can say that the choice of the dedualizing complex $B^{\bullet}=\mathbb{R} \Gamma_{I}(R)$ corresponds to the choice of the anti-equivalence $\mathbb{R} \operatorname{Hom}_{R}\left(-, B^{\bullet}\right)$ on the subcategory of compact objects in $\mathrm{D}\left(R-\bmod _{I \text {-tors }}\right)$ obtained as the restriction of the anti-equivalence $\mathbb{R} \operatorname{Hom}_{R}(-, R)$ on the category of compact objects in $\mathrm{D}(R-\mathrm{mod})$. 
Remark 5.9. We do not know how the definition of a dedualizing complex considered in this section relates to the one from Section 4. The difference lies in the conditions (iii). One might wish to replace the condition (iii) in the above definition with a more conventional finiteness condition by requiring, in the spirit of the condition (iii) of Section 4 , the cohomology $R$-modules of the complex $B^{\bullet}$ to have finitely generated submodules of elements annihilated by $I^{n}$ for all (or some) $n \geqslant 1$. However, the class of all $I$-torsion $R$-modules with finitely generated submodules of elements annihilated by $I^{n}$ does not seem to have good enough homological properties to make such a definition reasonable even for a Noetherian ring $R$ with a non-Artinian quotient ring $R / I$. Indeed, the following counterexample demonstrates that this class of $R$-modules is not closed under the passages to quotient modules (though it is, of course, preserved by the passages to arbitrary submodules).

Let $R=k[x, s]$ be the ring of polynomials in two variables over a field $k$ with the ideal $I=(s)$. Consider the $I$-torsion $R$-module $k\left[x, s, s^{-1}\right] / s k[x, s]$ and the $R$-submodule $M$ in it with the $k$-basis consisting of all the vectors $x^{j} s^{-i}$ with $i \leqslant j$. Then for any $n \geqslant 1$ the submodule of elements annihilated by $s^{n}$ in $M$ is a finitely generated free $k[x]$-module with the generators $1, x s^{-1}, \ldots, x^{n-1} s^{-n+1}$, but the quotient $R$-module $M / s M$ of the $R$-module $M$ is an infinite-dimensional $k$-vector space with the basis $x^{i} s^{-i}, i \geqslant 0$, where both $x$ and $s$ act by zero.

Hence even though the argument of Example 4.8 still shows that the derived functor of maximal submodule annihilated by $I^{n}$ takes the complex $B^{\bullet}=\mathbb{R} \Gamma_{I}(R)$ to a complex with finitely generated cohomology $R / I^{n}$-modules when the ring $R$ is Noetherian, no analogue of Lemmas 4.2 or 4.3 seems to be applicable in this case.

The next theorem is the main result of this section and this paper.

Theorem 5.10. Let $I$ be a weakly proregular finitely generated ideal in a commutative ring $R$. Then, given a dedualizing complex $B^{\bullet}$ for the ideal $I \subset R$, for any symbol $\star=\mathrm{b},+,-, \varnothing$, abs + , abs-, or abs there is an equivalence of derived categories $\mathrm{D}^{\star}\left(R-\bmod _{I \text {-tors }}\right) \simeq \mathrm{D}^{\star}\left(R-\bmod _{I \text {-ctra }}\right)$ provided by mutually inverse functors $\mathbb{R} \operatorname{Hom}_{R}\left(B^{\bullet},-\right)$ and $B^{\bullet} \otimes_{R}^{\mathbb{L}}-$.

Proof. As in the proof of Theorem 4.9, we assume for simplicity of notation that the complex $B^{\bullet}$ is concentrated in nonpositive cohomological degrees. Let $d$ be an integer greater or equal to both the projective and the contraflat dimension of $B^{\bullet}$ as a complex of $I$-torsion $R$-modules.

To construct the image of a complex of $I$-torsion $R$-modules $M^{\bullet}$ under the functor $\mathbb{R} \operatorname{Hom}_{R}\left(B^{\bullet},-\right)$, choose an exact sequence of complexes of $I$-torsion $R$-modules $0 \longrightarrow M^{\bullet} \longrightarrow J^{0, \bullet} \longrightarrow J^{1, \bullet} \longrightarrow \cdots$ with injective $I$-torsion $R$-modules $J^{j, i}$ (i. e., the $R$-modules $J^{j, i}$ should be injective objects in the abelian category $R$ - $\left.\bmod _{I \text {-tors }}\right)$. Applying the functor $\operatorname{Hom}_{R}\left(B^{\bullet},-\right)$ to every complex $0 \longrightarrow J^{0, i} \longrightarrow J^{1, i} \longrightarrow J^{2, i} \longrightarrow \cdots$ with $i \in \mathbb{Z}$, one obtains a nonnegatively graded complex of $I$-contramodule $R$-modules $0 \longrightarrow P^{0, i} \longrightarrow P^{1, i} \longrightarrow P^{2, i} \longrightarrow \cdots$. According to the projective dimension condition on the dedualizing complex $B^{\bullet}$, the complex $P^{\bullet}, i$ has zero cohomology modules 
at the cohomological degrees above $d$; so it quasi-isomorphic to its canonical truncation complex $\tau_{\leqslant d} P^{\bullet}, i$. By the definition, set the object $\mathbb{R} \operatorname{Hom}_{R}\left(B^{\bullet}, M^{\bullet}\right)$ in the derived category $\mathrm{D}^{\star}\left(R-\bmod _{I \text {-ctra }}\right)$ be represented by the total complex of the bicomplex $\tau_{\leqslant d} P^{\bullet}, \bullet$ concentrated in the cohomological degrees $0 \leqslant j \leqslant d$ and $i \in \mathbb{Z}$ (or, when the symbol $\star$ presumes bounded complexes, the bicomplex $P^{\bullet \bullet \bullet}$ is bounded in the respective sense along the grading $i$ ).

Similarly, to construct the image of a complex of $I$-contramodule $R$-modules $P$ under the functor $B^{\bullet} \otimes_{R}^{\mathbb{L}}-$, one has to choose an exact sequence of complexes of $I$-contramodule $R$-modules $\cdots \longrightarrow F^{-1, \bullet} \longrightarrow F^{0, \bullet} \longrightarrow P^{\bullet} \longrightarrow 0$ with projective $I$-contramodule $R$-modules $F^{j, i}$. Then one applies the functor $B^{\bullet} \otimes_{R}-$ to every complex $\cdots \longrightarrow F^{-2, i} \longrightarrow F^{-1, i} \longrightarrow F^{0, i} \longrightarrow 0$ with $i \in \mathbb{Z}$, obtaining a nonpositively graded complex of $I$-torsion $R$-modules $\cdots \longrightarrow M^{-2, i} \longrightarrow M^{-1, i} \longrightarrow M^{0, i} \longrightarrow 0$. According to the contraflat dimension condition on the complex $B^{\bullet}$, the complex $M^{\bullet}, i$ has zero cohomology modules at the cohomological degrees below $-d$; so it is quasi-isomorphic to its canonical truncation complex $\tau_{\geqslant-d} M^{\bullet, i}$. One sets the object $B^{\bullet} \otimes_{R}^{\mathbb{L}} P^{\bullet}$ in the derived category $\mathrm{D}^{\star}\left(R-\bmod _{I \text {-tors }}\right)$ to be represented by the total complex of the bicomplex $\tau_{\geqslant-d}\left(M^{\bullet} \bullet\right)$ concentrated in the cohomological degrees $-d \leqslant$ $j \leqslant 0$ and $i \in \mathbb{Z}$.

As in the proof of Theorem 4.9, these constructions of two derived functors are but particular cases of the construction of a derived functor of finite homological dimension elaborated in Appendix B. According to the results of Appendix B, the above constructions produce well-defined triangulated functors

$$
\mathbb{R} \operatorname{Hom}_{R}\left(B^{\bullet},-\right): \mathrm{D}^{\star}\left(R-\bmod _{I \text {-tors }}\right) \longrightarrow \mathrm{D}^{\star}\left(R-\bmod _{I \text {-ctra }}\right)
$$

and

$$
B^{\bullet} \otimes_{R}^{\mathbb{L}}-: \mathrm{D}^{\star}\left(R-\bmod _{I \text {-ctra }}\right) \longrightarrow \mathrm{D}^{\star}\left(R-\bmod _{I \text {-tors }}\right)
$$

for any derived category symbol $\star=b,+,-, \varnothing$, abs + , abs - , or abs. Moreover, the former functor is right adjoint to the latter one. All these assertions only depend on the condition (i) in the definition of a dedualizing complex.

It remains to prove that the adjunction morphisms are isomorphisms. Since the total complexes of finite acyclic complexes of complexes are absolutely acyclic, in order to check that the morphism $B^{\bullet} \otimes_{R}^{\mathbb{L}} \mathbb{R} \operatorname{Hom}_{R}\left(B^{\bullet}, M^{\bullet}\right) \longrightarrow M^{\bullet}$ is an isomorphism in the derived category $\mathrm{D}^{\star}\left(R-\bmod _{I \text {-tors }}\right)$ for all the $\star$-bounded complexes of $I$-torsion $R$-modules $M^{\bullet}$ it suffices to consider the case of a one-term complex $M^{\bullet}=M$ corresponding to a single $I$-torsion $R$-module $M$. Furthermore, since a morphism in $\mathrm{D}^{\mathrm{b}}\left(R-\bmod _{I \text {-tors }}\right)$ is an isomorphism whenever it is an isomorphism in $\mathrm{D}^{+}\left(R-\bmod _{I \text {-tors }}\right)$, one can view the one-term complex $M$ as an object of the derived category $\mathrm{D}^{+}\left(R-\bmod _{I \text {-tors }}\right)$ and replace it with an injective resolution $J^{\bullet}$ of the $R$-module $M$ in the abelian category $R-\bmod _{I \text {-tors }}$. Applying the same totalization argument to the complex $J^{\bullet}$, the question reduces to proving that the adjunction morphism $B^{\bullet} \otimes \frac{\mathbb{L}}{R} \mathbb{R} \operatorname{Hom}_{R}\left(B^{\bullet}, J\right) \longrightarrow J$ is an isomorphism in $\mathrm{D}^{\mathrm{b}}\left(R-\bmod _{I \text {-tors }}\right)$ for any injective $I$-torsion $R$-module $J$. 
One has $\mathbb{R} \operatorname{Hom}_{R}\left(B^{\bullet}, J\right)=\operatorname{Hom}_{R}\left(B^{\bullet}, J\right)$. Let $P^{\bullet}$ be a bounded above complex of projective $I$-contramodule $R$-modules endowed with a quasi-isomorphism of complexes of $I$-contramodule $R$-modules $P^{\bullet} \longrightarrow \operatorname{Hom}_{R}\left(B^{\bullet}, J\right)$, so $B^{\bullet} \otimes_{R}^{\mathbb{L}} \operatorname{Hom}_{R}\left(B^{\bullet}, J\right)=$ $B^{\bullet} \otimes_{R} P^{\bullet}$. We have to show that the natural morphism $B^{\bullet} \otimes_{R} P^{\bullet} \longrightarrow J$ is a quasiisomorphism of complexes of $I$-torsion $R$-modules. Let $\mathbf{s}$ be a finite sequence of elements generating the ideal $I \subset R$. By Lemma 1.1(c), it suffices to check that the induced morphism of complexes

$$
T_{n}^{\bullet}(R, \mathbf{s}) \otimes_{R} B^{\bullet} \otimes_{R} P^{\bullet} \longrightarrow T_{n}^{\bullet}(R, \mathbf{s}) \otimes_{R} J
$$

is a quasi-isomorphism for every $n \geqslant 1$ (as the complex of flat $R$-modules $C_{\mathbf{s}}(R)^{\sim}$ is quasi-isomorphic to the complex of free $R$-modules $T^{\bullet}(R, \mathbf{s})$, which is the inductive limit of complexes of finitely generated free $R$-modules $\left.T_{n}^{\bullet}(R, \mathbf{s})\right)$.

By the condition (iii) together with Lemma 5.4(a) and Proposition 5.1, there exists a finite complex of finitely generated projective $R$-modules $L$ together with a quasiisomorphism of complexes of $R$-modules $L^{\bullet} \longrightarrow T_{n}^{\bullet}(R, \mathbf{s}) \otimes_{R} B^{\bullet}$. By Lemma 5.4(c), the induced morphism of complexes of $R$-modules

$$
L^{\bullet} \otimes_{R} P^{\bullet} \longrightarrow T_{n}^{\bullet}(R, \mathbf{s}) \otimes_{R} B^{\bullet} \otimes_{R} P^{\bullet}
$$

is a quasi-isomorphism. So is the morphism of complexes of $R$-modules $L \bullet \otimes_{R} P^{\bullet} \longrightarrow$ $L^{\bullet} \otimes_{R} \operatorname{Hom}_{R}\left(B^{\bullet}, J\right)$. Hence it remains to check that the morphism

$$
L \bullet \otimes_{R} \operatorname{Hom}_{R}\left(B^{\bullet}, J\right) \longrightarrow T_{n}^{\bullet}(R, \mathbf{s}) \otimes_{R} J
$$

induced by the morphism $L^{\bullet} \longrightarrow T_{n}^{\bullet}(R, \mathbf{s}) \otimes_{R} B^{\bullet}$ is a quasi-isomorphism.

One has $L^{\bullet} \otimes_{R} \operatorname{Hom}_{R}\left(B^{\bullet}, J\right) \simeq \operatorname{Hom}_{R}\left(\operatorname{Hom}_{R}\left(L^{\bullet}, B^{\bullet}\right), J\right)$. By Theorem 5.7, the natural morphism of complexes of $R$-modules

$$
K_{\bullet}^{n}(R, \mathbf{s})=\operatorname{Hom}_{R}\left(T_{n}^{\bullet}(R, \mathbf{s}), R\right) \longrightarrow \operatorname{Hom}_{R}\left(L^{\bullet}, B^{\bullet}\right)
$$

is a quasi-isomorphism. Applying Lemma 5.5(b), we conclude that the induced morphism of complexes

$$
\operatorname{Hom}_{R}\left(\operatorname{Hom}_{R}\left(L^{\bullet}, B^{\bullet}\right), J\right) \longrightarrow \operatorname{Hom}_{R}\left(K_{\bullet}^{n}(R, \mathbf{s}), J\right) \simeq T_{n}^{\bullet}(R, \mathbf{s}) \otimes_{R} J
$$

is a quasi-isomorphism, too.

Similarly, in order to prove that the adjunction morphism $P^{\bullet} \longrightarrow \mathbb{R} \operatorname{Hom}_{R}\left(B^{\bullet}\right.$, $\left.B^{\bullet} \otimes_{R}^{\mathbb{L}} P^{\bullet}\right)$ is an isomorphism in the derived category $\mathrm{D}^{\star}\left(R\right.$ - $\left.\bmod _{I \text {-ctra }}\right)$ for any

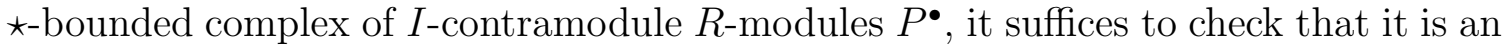
isomorphism in $\mathrm{D}^{\mathrm{b}}\left(R\right.$ - $\left.\bmod _{I \text {-ctra }}\right)$ for any free $I$-contramodule $R$-module $F=\mathfrak{R}[[X]]$ viewed as a one-term complex in $\mathrm{D}^{\mathrm{b}}\left(R-\bmod _{I \text {-ctra }}\right)$. One has $B^{\bullet} \otimes_{R}^{\mathbb{L}} \mathfrak{R}[[X]] \simeq$ $B^{\bullet} \otimes_{R} R[X]=B^{\bullet}[X]$. Let $B^{\bullet} \longrightarrow E^{\bullet}$ and $B^{\bullet}[X] \longrightarrow E_{X}^{\bullet}$ be quasi-isomorphisms from the complexes $B^{\bullet}$ and $B^{\bullet}[X]$ to bounded below complexes of injective objects in $R-\bmod _{I \text {-tors }}$. We have to show that the natural morphism $\mathfrak{R}[[X]] \longrightarrow \operatorname{Hom}_{R}\left(B^{\bullet}, E_{X}^{\bullet}\right)$ is a quasi-isomorphism of complexes of $R$-modules.

By Lemma 1.1(c), the morphism of complexes of $I$-torsion $R$-modules $T^{\bullet}(R, \mathbf{s}) \otimes_{R}$ $B^{\bullet} \longrightarrow B^{\bullet}$ is a quasi-isomorphism. Hence the induced morphism

$$
\operatorname{Hom}_{R}\left(B^{\bullet}, E_{X}^{\bullet}\right) \longrightarrow \operatorname{Hom}_{R}\left(T^{\bullet}(R, \mathbf{s}) \otimes_{R} B^{\bullet}, E_{X}^{\bullet}\right)
$$


is a quasi-isomorphism, too. The complex in the target of this morphism is the projective limit of the projective system of complexes $\operatorname{Hom}_{R}\left(T_{n}^{\bullet}(R, \mathbf{s}) \otimes_{R} B^{\bullet}, E_{X}^{\bullet}\right)$ and termwise surjective morphisms between them. By the condition (iii), every complex $T_{n}^{\bullet}(R, \mathbf{s}) \otimes_{R} B^{\bullet}$ is a compact object of the derived category $\mathrm{D}\left(R-\bmod _{I \text {-tors }}\right)$. Hence the $X$-indexed family of morphisms of complexes $E^{\bullet} \longrightarrow E_{X}^{\bullet}$ corresponding to the embeddings $B^{\bullet} \longrightarrow B^{\bullet}[X]$ induces a quasi-isomorphism

$$
\operatorname{Hom}_{R}\left(T_{n}^{\bullet}(R, \mathbf{s}) \otimes_{R} B^{\bullet}, E^{\bullet}\right)[X] \longrightarrow \operatorname{Hom}_{R}\left(T_{n}^{\bullet}(R, \mathbf{s}) \otimes_{R} B^{\bullet}, E_{X}^{\bullet}\right) .
$$

Furthermore, one has

$$
\operatorname{Hom}_{R}\left(T_{n}^{\bullet}(R, \mathbf{s}) \otimes_{R} B^{\bullet}, E^{\bullet}\right) \simeq \operatorname{Hom}_{R}\left(T_{n}^{\bullet}(R, \mathbf{s}), \operatorname{Hom}_{R}\left(B^{\bullet}, E^{\bullet}\right)\right) .
$$

By the condition (ii), the natural morphism $\mathfrak{R} \longrightarrow \operatorname{Hom}_{R}\left(B^{\bullet}, E^{\bullet}\right)$ is a quasiisomorphism, hence so is the induced morphism

$$
\operatorname{Hom}_{R}\left(T_{n}^{\bullet}(R, \mathbf{s}), \mathfrak{R}\right) \longrightarrow \operatorname{Hom}_{R}\left(T_{n}^{\bullet}(R, \mathbf{s}), \operatorname{Hom}_{R}\left(B^{\bullet}, E^{\bullet}\right)\right) .
$$

By Lemma 5.4(a-b), the morphism of complexes

$$
\operatorname{Hom}_{R}\left(T_{n}^{\bullet}(R, \mathbf{s}), R\right) \longrightarrow \operatorname{Hom}_{R}\left(T_{n}^{\bullet}(R, \mathbf{s}), \mathfrak{R}\right)
$$

induced by the completion morphism $R \longrightarrow \mathfrak{R}$ is a quasi-isomorphism, too. Passing to the composition and taking the direct sum over $X$, we obtain a quasi-isomorphism of complexes of $R$-modules

$$
\operatorname{Hom}_{R}\left(T_{n}^{\bullet}(R, \mathbf{s}), R[X]\right) \longrightarrow \operatorname{Hom}_{R}\left(T_{n}^{\bullet}(R, \mathbf{s}) \otimes_{R} B^{\bullet}, E^{\bullet}\right)[X]
$$

for every $n \geqslant 1$.

Both sides of the quasi-isomorphisms (7) and (8) naturally form projective systems of complexes and termwise surjective morphisms between them, and the quasiisomorphisms form commutative diagrams with the morphisms in the projective systems. Therefore, the induced morphisms between the projective limits are also quasiisomorphisms. Furthermore, the complexes $\operatorname{Hom}_{R}\left(T_{n}^{\bullet}(R, \mathbf{s}), R[X]\right)$ have $I$-torsion cohomology modules, so the natural morphism $R[X] \longrightarrow \operatorname{Hom}_{R}\left(T_{n}^{\bullet}(R, \mathbf{s}), R[X]\right)$ extends naturally to a cohomology morphism

$$
\mathfrak{R}[[X]] \longrightarrow H^{0} \operatorname{Hom}_{R}\left(T_{n}^{\bullet}(R, \mathbf{s}), R[X]\right) .
$$

Due to the weak proregularity condition, after the passage to the projective limit this provides a cohomology morphism

$$
\mathfrak{R}[[X]] \longrightarrow H^{0} \lim _{n} \operatorname{Hom}_{R}\left(T_{n}^{\bullet}(R, \mathbf{s}), R[X]\right)
$$

forming a commutative diagram with the cohomology morphisms induced by the morphism of complexes $\mathfrak{R}[[X]] \longrightarrow \operatorname{Hom}_{R}\left(B^{\bullet}, E_{X}^{\bullet}\right)$, the morphism of complexes (6), and the projective limits of the morphisms of complexes (7-8).

Finally, by Lemma 5.3(c) the morphism of complexes

$$
\lim _{n} \operatorname{Hom}_{R}\left(T_{n}^{\bullet}(R, \mathbf{s}), R[X]\right) \simeq \operatorname{Hom}_{R}\left(T^{\bullet}(R, \mathbf{s}), R[X]\right) \longrightarrow \operatorname{Hom}_{R}\left(T^{\bullet}(R, \mathbf{s}), \mathfrak{R}[[X]]\right)
$$


induced by the completion morphism $R[X] \longrightarrow \mathfrak{R}[[X]]$ is a quasi-isomorphism, while by Lemma 2.2(c) the morphism

$$
\mathfrak{R}[[X]] \longrightarrow \operatorname{Hom}_{R}\left(T^{\bullet}(R, \mathbf{s}), \mathfrak{R}[[X]]\right)
$$

is a quasi-isomorphism. Comparing these observations finishes the proof.

\section{Appendix A. Exotic Derived Categories}

This appendix, consisting almost entirely of the definitions, is included for the benefit of the reader who may have no other sources handy when looking into this paper. Detailed expositions can be found in [21, 22, 7]; and the most relevant source for our present purposes is [24, Appendix A].

Given an additive category $A$, we denote by $\operatorname{Hot}(A)$ the homotopy category of complexes over $A$, by $\operatorname{Hot}^{+}(A)$ its full subcategory of bounded below complexes, by $\operatorname{Hot}^{-}(A) \subset \operatorname{Hot}(A)$ the full subcategory of bounded above complexes, and by $\operatorname{Hot}^{b}(A)$ the full subcategory of complexes bounded on both sides.

For a general discussion of exact categories, we refer to the overview paper [3]. A sequence of objects and morphisms $E \longrightarrow F \longrightarrow G$ in an exact category $\mathrm{E}$ is said to be exact (at the term $F$ ) if it is composed of an admissible epimorphism $E \longrightarrow S$, a short exact sequence $0 \longrightarrow S \longrightarrow F \longrightarrow T \longrightarrow 0$, and an admissible monomorphism $T \longrightarrow G$. A complex $E^{\bullet}$ in an exact category $\mathrm{E}$ is said to be acyclic if it is homotopy equivalent to an exact complex, or equivalently, if it is a direct summand of an exact complex. For any symbol $\star=b,+,-$, or $\varnothing$, the quotient category of the homotopy category $\operatorname{Hot}^{\star}(\mathrm{E})$ by its thick subcategory of acyclic complexes is called the (conventional) derived category of $E$ and denoted by $D^{\star}(E)$.

The exotic derived category symbols are $\star=$ abs + , abs - , co, ctr, or abs. The related exotic derived category $D^{\star}(E)$ is defined as a certain quotient category of

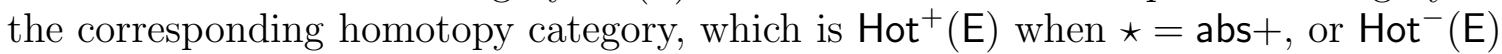
when $\star=$ abs - , or Hot $(E)$ in the remaining cases $\star=c o$, ctr, or abs. It is sometimes convenient to denote simply by $\operatorname{Hot}^{\star}(\mathrm{E})$ the (respectively bounded or unbounded) homotopy category corresponding to an exotic symbol $\star$.

A short exact sequence of complexes in an exact category $E$ can be viewed as a bicomplex with three rows. As such, it has a total complex. A complex in an exact category $\mathrm{E}$ is called absolutely acyclic if it belongs to the minimal thick subcategory of the homotopy category $\operatorname{Hot}(\mathrm{E})$ containing the totalizations of all the short exact sequences of complexes in $E$. The quotient category of the homotopy category $\operatorname{Hot}(E)$ by its thick subcategory of absolutely acyclic complexes is denoted by $D^{\text {abs }}(E)$ and called the absolute derived category of an exact category $\mathrm{E}$.

The definitions of the absolute derived categories $D^{\text {abs }+}(E)$ and $D^{\text {abs- }}(E)$ of bounded above or below complexes in an exact category $E$ are similar. $A \star$-bounded complex in $\mathrm{E}$ is said to be absolutely acyclic with respect to the class of $\star$-bounded complexes if it belongs to the minimal thick subcategory of the homotopy category $\operatorname{Hot}^{\star}(\mathrm{E})$ containing the totalizations of all the short exact sequences of $\star$-bounded complexes 
in E. Given a symbol $\star=$ abs + or abs - , the quotient category of the homotopy category $\operatorname{Hot}^{\star}(E)$ by its thick subcategory of complexes absolutely acyclic with respect to the class of $\star$-bounded complexes is denoted by $D^{\star}(E)$.

Any bounded acyclic complex is absolutely acyclic (with respect to the class of bounded complexes). Any bounded above complex that is absolutely acyclic is also absolutely acyclic with respect to the class of bounded above complexes; and any bounded below complex that is absolutely acyclic is also absolutely acyclic with respect to the class of bounded below complexes [24, Lemma A.1.1].

Assume that arbitrary infinite direct sums exist in the exact category $E$ and the class of short exact sequences is closed under infinite direct sums. Then a complex in the category $\mathrm{E}$ is called coacyclic if it belongs to the minimal triangulated subcategory of $\operatorname{Hot}(\mathrm{E})$ containing the absolutely acyclic complexes and closed under infinite direct sums. The quotient category of the homotopy category Hot(E) by its thick subcategory of coacyclic complexes is denoted by $\mathrm{D}^{\mathrm{co}}(\mathrm{E})$ and called the coderived category of the exact category $\mathrm{E}$.

Similarly, assume that arbitrary infinite products exist in the exact category $\mathrm{E}$ and the class of short exact sequences is closed under infinite products. Then a complex in the category $\mathrm{E}$ is called contraacyclic if it belongs to the minimal triangulated subcategory of $\operatorname{Hot}(\mathrm{E})$ containing the absolutely acyclic complexes and closed under infinite products. The quotient category of the homotopy category $\operatorname{Hot}(\mathrm{E})$ by its thick subcategory of contraacyclic complexes is denoted by $\mathrm{D}^{\mathrm{ctr}}(\mathrm{E})$ and called the contraderived category of the exact category $\mathrm{E}$.

Any coacyclic complex in an exact category with exact functors of infinite direct sum is acyclic, and any contraacyclic complex in an exact category with exact functors of infinite product is acyclic, but the converse is not generally true [22, Examples 3.3]. So the coderived and the contraderived categories are "larger" than the conventional unbounded derived category; and the absolute derived category is larger still.

Any bounded below acyclic complex in an exact category is coacyclic $[21$, Lemma 2.1]. Any bounded above acyclic complex in an exact category is contraacyclic [21, Lemma 4.1]. Any acyclic complex in an exact category of finite homological dimension is absolutely acyclic [21, Remark 2.1].

The conventional derived categories (with the symbols,+- , or $\varnothing$ ) are also known as derived categories of the first kind. The coderived, contraderived, and absolute derived categories (with the symbols abs+, abs-, co, ctr, or abs) are known as derived categories of the second kind. The bounded derived category $\mathrm{D}^{\mathrm{b}}(\mathrm{E})$ of an exact category $\mathrm{E}$ can be thought of as belonging to both classes at the same time.

\section{Appendix B. Derived Functors of Finite Homological Dimension}

The constructions of derived functors of finite homological dimension between various derived categories $D^{\star}$ in Sections $1-2$ are based on the technique of $[24$, Section A.5], which is not sufficient for the purposes of Sections 4-5. The more sophisticated technique required there is developed in this appendix. 
For any additive category $\mathrm{E}$, we denote by $\mathrm{C}^{+}(\mathrm{E})$ the $\mathrm{DG}$-category of bounded below complexes in $\mathrm{E}$. The additive category of bounded below complexes in $\mathrm{E}$ and closed morphisms of degree zero between them will be denoted by the same symbol $\mathrm{C}^{+}(\mathrm{E})$; it will be clear from the context which category structure on $\mathrm{C}^{+}(\mathrm{E})$ is presumed. When $E$ is an exact category, the full subcategory $C^{\geqslant 0}(E) \subset C^{+}(E)$ of all the complexes $0 \longrightarrow E^{0} \longrightarrow E^{1} \longrightarrow E^{2} \longrightarrow \cdots$ in $\mathrm{E}$ (and closed morphisms of degree zero between them) has a natural exact category structure where a short sequence of complexes is exact if and only if it is termwise exact in $\mathrm{E}$.

Let $A$ be an exact category and $\mathrm{J} \subset \mathrm{A}$ be a full subcategory, closed under extensions and the passages to the cokernels of admissible monomorphisms in $A$ and such that any object of $A$ is the source of an admissible monomorphism into an object of $J$. Being closed under extensions, the full subcategory $J$ inherits the exact category structure of the ambient category A. Notice that a closed morphism in $\mathrm{C}^{+}(\mathrm{J})$ is a quasi-isomorphism of complexes in the exact category $\mathrm{J}$ if and only if it is a quasiisomorphism of complexes in A.

Let $C_{A}^{\geqslant 0}(J)$ denote the full subcategory in $C \geqslant 0(J)$ consisting of all the complexes $0 \longrightarrow J^{0} \longrightarrow J^{1} \longrightarrow \cdots$ in $\mathrm{J}$ for which there exists an object $A \in \mathrm{A}$ together with a morphism $A \longrightarrow J^{0}$ such that the sequence $0 \longrightarrow A \longrightarrow J^{0} \longrightarrow J^{1} \longrightarrow \cdots$ is exact in the category $A$. By the definition, one has $C_{A}^{\geqslant 0}(J)=C \geqslant 0(J) \cap C_{A}^{\geqslant 0}(A) \subset C \geqslant 0(A)$. The full subcategory $C_{A}^{\geqslant 0}(J)$ is closed under extensions and the passages to the cokernels of admissible monomorphisms in $C^{\geqslant 0}(J)$, so it inherits the exact category structure.

Let $\mathrm{B}$ be another exact category; suppose that it contains the images of idempotent endomorphisms of its objects. Let $d \geqslant 0$ be an integer. Denote by $C^{\geqslant 0}(B)^{\leqslant d} \subset C \geqslant 0(B)$ the full subcategory consisting of all the complexes $0 \longrightarrow B^{0} \longrightarrow B^{1} \longrightarrow \cdots$ in $\mathrm{B}$ such that the sequence $B^{d} \longrightarrow B^{d+1} \longrightarrow B^{d+2} \longrightarrow \cdots$ is exact in B. In particular, one has $C^{\geqslant 0}(B)^{\leqslant 0}=C_{B}^{\geqslant 0}(B)$. Being closed under extensions and the passages to the cokernels of admissible monomorphisms, the full subcategory $C \geqslant 0(B) \leqslant d$ inherits the exact category structure of the category $C^{\geqslant 0}(B)$. Denote also by $C^{[0, d]}(B)$ the exact category of finite complexes $B^{0} \longrightarrow \cdots \longrightarrow B^{d}$ in $\mathrm{B}$. Then there is an exact functor of canonical truncation $\tau_{\leqslant d}: \mathrm{C}^{\geqslant 0}(\mathrm{~B})^{\leqslant d} \longrightarrow \mathrm{C}^{[0, d]}(\mathrm{B})$.

Suppose that we are given a DG-functor $\Psi: \mathrm{C}^{+}(\mathrm{J}) \longrightarrow \mathrm{C}^{+}(\mathrm{B})$ taking quasiisomorphisms of complexes belonging to $\mathrm{C}_{A}^{\geqslant 0}(\mathrm{~J})$ to quasi-isomorphisms of complexes in the exact category B. Suppose further that the restriction of the functor $\Psi$ to the subcategory $\mathrm{C}_{\mathrm{A}}^{\geqslant 0}(\mathrm{~J}) \subset \mathrm{C}^{+}(\mathrm{J})$ is an exact functor between exact categories $\Psi: \mathrm{C}_{\mathrm{A}}(\mathrm{J}) \longrightarrow \mathrm{C}^{\geqslant 0}(\mathrm{~B}) \leqslant d$. Composing the restriction of the functor $\Psi$ with the functor of canonical truncation, we obtain an exact functor

$$
\tau_{\leqslant d} \Psi: \mathrm{C}_{\mathrm{A}}^{\geqslant 0}(\mathrm{~J}) \longrightarrow \mathrm{C}^{[0, d]}(\mathrm{B}) .
$$

Our aim is to construct the right derived functor

$$
\mathbb{R} \Psi: \mathrm{D}^{\star}(\mathrm{A}) \longrightarrow \mathrm{D}^{\star}(\mathrm{B})
$$

acting between any bounded or unbounded, conventional or absolute derived categories $\mathrm{D}^{\star}$ with the symbol $\star=\mathrm{b},+,-, \varnothing$, abs + , abs-, or abs (see Appendix A or [24, Section A.1] for the definitions and [22, Section 3] for a discussion). 
When the exact categories $A$ and $B$ have exact functors of infinite direct sum, the full subcategory $\mathrm{J} \subset \mathrm{A}$ is closed under infinite direct sums, and the functor $\Psi$ preserves infinite direct sums, there will be also the derived functor $\mathbb{R} \Psi$ acting between the coderived categories $D^{c o}$ of the exact categories A and B. Similarly, when the exact categories $B$ and $A$ have exact functors of infinite product, the full subcategory $J \subset A$ is closed under infinite products, and the functor $\Psi$ preserves infinite products, we will also have the derived functor $\mathbb{R} \Psi$ acting between the contraderived categories $D^{\text {ctr }}$ of the exact categories $A$ and $B$.

We start with a couple of somewhat tedious but rather straightforward lemmas.

Lemma B.1. (a) For any object $E$ in the category A there exists an exact sequence $0 \longrightarrow E \longrightarrow J^{0} \longrightarrow J^{1} \longrightarrow J^{2} \longrightarrow \cdots$ in the category A with the objects $J^{j}$ belonging to the full subcategory $\mathrm{J} \subset \mathrm{A}$.

(b) For any two morphisms $E \longrightarrow G$ and $F \longrightarrow G$ and two exact sequences $0 \longrightarrow E \longrightarrow K^{0} \longrightarrow K^{1} \longrightarrow \cdots$ and $0 \longrightarrow F \longrightarrow L^{0} \longrightarrow L^{1} \longrightarrow \cdots$ in the category A there exists an exact sequence $0 \longrightarrow G \longrightarrow J^{0} \longrightarrow J^{1} \longrightarrow \cdots$ in the category A together with two morphisms of complexes $K^{\bullet} \longrightarrow J^{\bullet}$ and $L^{\bullet} \longrightarrow J^{\bullet}$ forming a commutative diagram with the morphisms $E \longrightarrow G, F \longrightarrow G, E \longrightarrow K^{\bullet}$, and $F \longrightarrow L^{\bullet}$ and such that the objects $J^{j}$ belong to the full subcategory $\mathrm{J} \subset \mathrm{A}$.

(c) For any exact sequence $0 \longrightarrow E \longrightarrow K^{0} \longrightarrow K^{1} \longrightarrow \cdots$ and any complex $0 \longrightarrow L^{0} \longrightarrow L^{1} \longrightarrow L^{2} \longrightarrow \cdots$ in the category $\mathrm{A}$, and for any morphism of complexes $K^{\bullet} \longrightarrow L^{\bullet}$ with a vanishing composition $E \longrightarrow K^{\bullet} \longrightarrow L^{\bullet}$, there exists a complex $0 \longrightarrow J^{0} \longrightarrow J^{1} \longrightarrow J^{2} \longrightarrow \cdots$ in the category J together with a quasiisomorphism $L^{\bullet} \longrightarrow J^{\bullet}$ of complexes in the category A such that the composition $K^{\bullet} \longrightarrow L^{\bullet} \longrightarrow J^{\bullet}$ is a contractible morphism of complexes.

(d) For any short exact sequence $0 \longrightarrow E^{1} \longrightarrow E^{2} \longrightarrow E^{3} \longrightarrow 0$ in the category A there exists an exact sequence of short exact sequences $0 \longrightarrow E^{\bullet} \longrightarrow J^{\bullet, 0} \longrightarrow$ $J^{\bullet, 1} \longrightarrow J^{\bullet, 2} \longrightarrow \cdots$ in the category A with the objects $J^{k, j}$ belonging to the full subcategory $\mathrm{J} \subset \mathrm{A}$.

Proof. Part (a) follows immediately by induction from the condition that any object of $A$ is the source of an admissible monomorphism into an object of $\mathrm{J}$. To prove part (b), one also proceeds step by step, starting from choosing an admissible monomorphism from the fibered coproduct of the objects $G$ and $K^{0} \oplus L^{0}$ over $E \oplus F$ into an object $J^{0} \in \mathrm{J}$, then applying the same construction to the cokernels of the morphisms $E \longrightarrow K^{0}, F \longrightarrow L^{0}$, and $G \longrightarrow J^{0}$ and the admissible monomorphisms from the former two to the objects $K^{1}$ and $L^{1}$, etc.

The proof of part (c) is also a step-by-step construction procedure. Let $Z^{j}$ denote the images of the morphisms $K^{j-1} \longrightarrow K^{j}$ in the exact sequence $0 \longrightarrow E \longrightarrow K^{0} \longrightarrow$ $K^{1} \longrightarrow \cdots$. Then the morphism $K^{0} \longrightarrow L^{0}$ factorizes through the admissible epimorphism $K^{0} \longrightarrow Z^{1}$, so there is a morphism $Z^{1} \longrightarrow L^{0}$. Consider the fibered coproduct of the objects $K^{1}$ and $L^{0}$ over the object $Z^{1}$ in the category A and choose an admissible monomorphism from it into an object $J^{0} \in J$. Then there are a natural admissible monomorphism $L^{0} \longrightarrow J^{0}$ and a natural morphism $K^{1} \longrightarrow J^{0}$. Denote by $M^{1}$ the fibered coproduct of the objects $J^{0}$ and $L^{1}$ over the object $L^{0}$ 
in the category $\mathrm{A}$. Then there are a natural admissible monomorphism $L^{1} \longrightarrow M^{1}$ and natural morphisms $J^{0} \longrightarrow M^{1} \longrightarrow L^{2}$ with a vanishing composition in the category $\mathrm{A}$. The difference of the compositions $K^{1} \longrightarrow L^{1} \longrightarrow M^{1}$ and $K^{1} \longrightarrow$ $J^{0} \longrightarrow M^{1}$ is annihilated by the composition with the morphism $K^{0} \longrightarrow K^{1}$, so it factorizes through the admissible epimorphism $K^{1} \longrightarrow Z^{2}$, providing a morphism $Z^{2} \longrightarrow M^{1}$. Consider the fibered coproduct of the objects $K^{2}$ and $M^{1}$ over the object $Z^{2}$ in the category $\mathrm{A}$ and choose an admissible monomorphism from it into an object $J^{1} \in \mathrm{J}$. Then there are a natural admissible monomorphism $M^{1} \longrightarrow J^{1}$ and a morphism $K^{2} \longrightarrow J^{1}$ in the category A. The composition $J^{0} \longrightarrow M^{1} \longrightarrow J^{1}$ provides a morphism $J^{0} \longrightarrow J^{1}$ in the category $\mathrm{J}$. Denote by $M^{2}$ the fibered coproduct of the objects $J^{1}$ and $L^{2}$ over the object $M^{1}$ in the category A, etc.

To check part (d), one applies part (a) to the exact category of short exact sequences in $A$ and its full subcategory of short exact sequences in J. It suffices to show that the short exact sequence $0 \longrightarrow E^{1} \longrightarrow E^{2} \longrightarrow E^{3} \longrightarrow 0$ is the source of an admissible monomorphism into a short exact sequence of objects from $\mathrm{J}$. For this purpose, one picks admissible monomorphisms $E^{2} \longrightarrow J^{1}$ and $E^{3} \longrightarrow J^{3}$ into objects $J^{1}, J^{3} \in \mathrm{J}$; then there is an admissible monomorphism from our short exact sequence to the split short exact sequence $0 \longrightarrow J^{1} \longrightarrow J^{1} \oplus J^{3} \longrightarrow J^{3} \longrightarrow 0$.

Lemma B.2. (a) For any complex $E^{\bullet}$ in the category A there exists an exact sequence of complexes

$$
0 \longrightarrow E^{\bullet} \longrightarrow J^{0, \bullet} \longrightarrow J^{1, \bullet} \longrightarrow J^{2, \bullet} \longrightarrow \cdots
$$

in the category $\mathrm{A}$ with the objects $J^{j, i}$ belonging to the full subcategory $\mathrm{J} \subset \mathrm{A}$.

(b) For any two morphisms of complexes $E^{\bullet} \longrightarrow G^{\bullet}$ and $F^{\bullet} \longrightarrow G^{\bullet}$ and two exact sequences of complexes $0 \longrightarrow E^{\bullet} \longrightarrow K^{0, \bullet} \longrightarrow K^{1, \bullet} \longrightarrow \cdots$ and $0 \longrightarrow F^{\bullet} \longrightarrow$ $L^{0, \bullet} \longrightarrow L^{1, \bullet} \longrightarrow \cdots$ in the category $\mathrm{A}$ there exists an exact sequence of complexes $0 \longrightarrow G^{\bullet} \longrightarrow J^{0} \bullet \longrightarrow J^{1, \bullet} \longrightarrow \cdots$ in the category A together with two morphisms of bicomplexes $K^{\bullet \bullet \bullet} \longrightarrow J^{\bullet, \bullet}$ and $L^{\bullet \bullet \bullet} \longrightarrow J^{\bullet \bullet \bullet}$ forming a commutative diagram with the morphisms $E^{\bullet} \longrightarrow G^{\bullet}, F^{\bullet} \longrightarrow G^{\bullet}, E^{\bullet} \longrightarrow K^{\bullet \bullet \bullet}$, and $F^{\bullet} \longrightarrow L^{\bullet \bullet}$ and such that the objects $J^{j, i}$ belong to the full subcategory $\mathrm{J} \subset \mathrm{A}$.

(c) For any exact sequence of complexes $0 \longrightarrow E^{\bullet} \longrightarrow K^{0, \bullet} \longrightarrow K^{1, \bullet} \longrightarrow \cdots$ and any complex of complexes $0 \longrightarrow L^{0, \bullet} \longrightarrow L^{1, \bullet} \longrightarrow L^{2, \bullet} \longrightarrow \cdots$ in the category

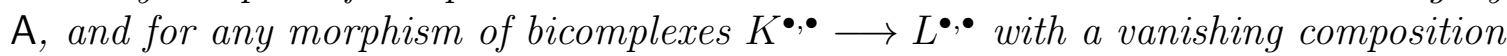
$E^{\bullet} \longrightarrow K^{\bullet \bullet \bullet} \longrightarrow L^{\bullet \bullet}$, there exists a complex of complexes $0 \longrightarrow J^{0, \bullet} \longrightarrow J^{1, \bullet} \longrightarrow$ $J^{2, \bullet} \longrightarrow \cdots$ in the category $\mathrm{J}$ together with a quasi-isomorphism $L^{\bullet \bullet \bullet} \longrightarrow J^{\bullet \bullet}$ of complexes along the first grading $j$ in the exact category of complexes along the second grading $i$ in the exact category A such that the composition $K^{\bullet} \bullet \longrightarrow L^{\bullet \bullet \bullet} \longrightarrow J^{\bullet \bullet \bullet}$ is a contractible morphism of complexes of complexes with a contracting homotopy $h: K^{j, i} \longrightarrow J^{j-1, i}$ defined for all $j \geqslant 0$ and $i \in \mathbb{Z}$.

(d) For any short exact sequence of complexes $0 \longrightarrow E^{1, \bullet} \longrightarrow E^{2, \bullet} \longrightarrow E^{3, \bullet} \longrightarrow 0$ in the category $\mathrm{A}$ there exists an exact sequence of short exact sequences of complexes $0 \longrightarrow E^{\bullet \bullet \bullet} \longrightarrow J^{\bullet, 0, \bullet} \longrightarrow J^{\bullet, 1, \bullet} \longrightarrow \cdots$ in the category A with the objects $J^{k, j, i}$ belonging to the full subcategory $\mathrm{J} \subset \mathrm{A}$. 
(e) For any exact complex $E^{\bullet}$ in the category $\mathrm{A}$ there exists an exact sequence of exact complexes $0 \longrightarrow E^{\bullet} \longrightarrow J^{0 \bullet} \longrightarrow J^{1, \bullet} \longrightarrow \cdots$ in the category A with the objects $J^{j, i}$ belonging to the full subcategory $\mathrm{J} \subset \mathrm{A}$ and the complexes $\mathrm{J}^{j, \bullet}$ exact in the exact category J.

(f) If in any of the parts (a-e) the original data of a complex, a pair of morphisms of complexes, a morphism of bicomplexes, or a short exact sequence of complexes consisted of (bi)complex(es) bounded above, below, or on both sides along the grading $i$, then the bicomplex or the short exact sequence of bicomplexes whose existence is asserted can be also chosen to be bounded on the respective side(s) along the grading $i$.

Proof. Parts (a-d) are obtained from parts (a-d) of Lemma B.1 by substituting the exact category of complexes in $A$ and its full subcategory of complexes in $\mathrm{J}$ in place of the exact category $A$ and its full subcategory J. One only has to show that any complex $E^{\bullet}$ in A is the source of an admissible monomorphism into a complex $J^{\bullet}$ with the terms from $J$. Here it suffices to pick admissible monomorphisms $E^{i} \longrightarrow K^{i}$ into some objects $K^{i} \in \mathrm{J}$ and set $J^{\bullet}$ to be the split exact complex with the terms $J^{i}=$ $K^{i} \oplus K^{i+1}$. The same construction of an admissible monomorphism of a complex in A into an exact complex in $\mathrm{J}$ allows to prove part (e). Part (f) is clear from the above argument; one only has to modify the construction of the admissible monomorphism $E^{\bullet} \longrightarrow J^{\bullet}$ slightly in the case of complexes bounded below or from both sides in order to keep the complex $J^{\bullet}$ within the boundaries of the complex $E^{\bullet}$.

Now we can construct the derived functor $\mathbb{R} \Psi$. Let $\star$ be one of the symbols $b,+$, ,$- \varnothing$, abs + , abs - , co, ctr, or abs; and let $C^{\star}(A)$ denote the category of (respectively bounded or unbounded) complexes (and closed morphisms of degree zero between them) in the category A. Given a complex $E^{\bullet} \in C^{\star}(A)$, we resolve it by a bicomplex $J^{\bullet \bullet \bullet}$ with the terms belonging to the subcategory $\mathrm{J} \subset \mathrm{A}$ as in Lemma B.2(a), apply the functor $\tau_{\leqslant d} \Psi$ to every complex $J^{j, \bullet}$ obtaining a bicomplex $\tau_{\leqslant d} \Psi\left(J^{\bullet}, \bullet\right)$ concentrated in the segment of degrees $[0, d]$ along the grading $j$, and totalize this bicomplex in order to obtain the complex $\mathbb{R} \Psi\left(E^{\bullet}\right) \in \mathrm{D}^{\star}(\mathrm{B})$. When the symbol $\star$ presumes bounded complexes, we choose the bicomplex $J^{\bullet \bullet \bullet}$ to be bounded on the respective side(s) along the grading $i$, as in Lemma B.2(f). This defines the action of the functor $\mathbb{R} \Psi$ on the objects $E^{\bullet}$ of the category of complexes $C^{\star}(A)$.

To construct the image of a morphism $E^{\bullet} \longrightarrow F^{\bullet}$ in the category of complexes $\mathrm{C}^{\star}(\mathrm{A})$ under the functor $\mathbb{R} \Psi$, one applies Lemma B.2(b) to the pair of morphisms of complexes $E^{\bullet} \longrightarrow F^{\bullet}$ and id $: F^{\bullet} \longrightarrow F^{\bullet}$ together with the chosen resolutions $E^{\bullet} \longrightarrow K^{\bullet \bullet \bullet}$ and $F^{\bullet} \longrightarrow L^{\bullet \bullet \bullet}$ of the complexes $E^{\bullet}$ and $F^{\bullet}$ by bicomplexes $K^{\bullet \bullet \bullet}$ and $L^{\bullet, \bullet}$ in the exact category $\mathrm{J}$. Then one uses the condition that the functor $\Psi$ takes quasi-isomorphisms in $C_{A}^{\geqslant 0}(J)$ to quasi-isomorphisms in $C \geqslant 0(B)$ in order to conclude that the induced morphism between the totalizations of the bicomplexes $\tau_{\leqslant d} \Psi\left(L^{\bullet, \bullet}\right)$ and $\tau_{\leqslant d} \Psi\left(J^{\bullet \bullet \bullet}\right)$ has an absolutely acyclic cone.

Proving that the morphism $\mathbb{R} \Psi\left(E^{\bullet}\right) \longrightarrow \mathbb{R} \Psi\left(F^{\bullet}\right)$ in the derived category $\mathrm{D}^{\star}(\mathrm{B})$ obtained in this way depends only on the original morphism $E^{\bullet} \longrightarrow F^{\bullet}$ in the category $\mathrm{C}^{\star}(\mathrm{A})$ and not on any additional choices, and also that compositions of morphisms in the category $D^{\star}(B)$ are assigned to compositions of morphisms in the category 
of complexes $C^{\star}(A)$, reduces to checking that a morphism of resolving bicomplexes $K^{\bullet \bullet \bullet} \longrightarrow L^{\bullet \bullet \bullet}$ in the category $\mathrm{J}$ forming a commutative diagram with a zero morphism of complexes $E^{\bullet} \longrightarrow F^{\bullet}$ in the category A induces a vanishing morphism between the totalizations of bicomplexes $\tau_{\leqslant d} \Psi\left(K^{\bullet, \bullet}\right) \longrightarrow \tau_{\leqslant d} \Psi\left(L^{\bullet \bullet \bullet}\right)$ in the derived category $\mathrm{D}^{\star}(\mathrm{B})$. Here one has to apply Lemma B.2(c) and recall that the functor $\Psi: \mathrm{C}^{+}(\mathrm{J}) \longrightarrow \mathrm{C}^{+}(\mathrm{B})$, having a DG-functor structure, transforms contracting homotopies to contracting homotopies and preserves the equations they may satisfy.

A morphism of complexes in the category $A$ is homotopic to zero if and only if it factorizes through a cone of an identity morphism of complexes, i. e., through a complex of the form $\cdots \longrightarrow E^{i-1} \oplus E^{i} \longrightarrow E^{i} \oplus E^{i+1} \longrightarrow \cdots$. Complexes of this form are easily seen to be taken to zero by the functor $\mathbb{R} \Psi$, which is therefore a well-defined functor from the homotopy category $\operatorname{Hot}^{\star}(A)$ to the derived category $D^{\star}(B)$.

Checking that the functor $\mathbb{R} \Psi: \operatorname{Hot}^{\star}(A) \longrightarrow D^{\star}(B)$ so obtained is triangulated reduces to another application of Lemma B.2(b), guaranteeing any closed morphism of complexes $E^{\bullet} \longrightarrow F^{\bullet}$ in the category $A$ can be included into a commutative diagram with resolution morphisms $E^{\bullet} \longrightarrow K^{\bullet \bullet \bullet}$ and $F^{\bullet} \longrightarrow L^{\bullet \bullet \bullet}$, and a morphism of resolving bicomplexes $K^{\bullet, \bullet} \longrightarrow L^{\bullet \bullet \bullet}$ in the category J. Since the cone of the closed morphism of bicomplexes $K^{\bullet \bullet \bullet} \longrightarrow L^{\bullet \bullet \bullet}$ (taken along the grading $i$ ) is a resolution of the cone of the closed morphism $E^{\bullet} \longrightarrow F^{\bullet}$, and the functor $\Psi$, being a DG-functor, preserves the cones of closed morphisms, the functor $\mathbb{R} \Psi$ takes cones to cones.

It remains to show that the functor $\mathbb{R} \Psi$ factorizes through the derived category $D^{\star}(A)$. Here one has to consider derived categories of the first and of the second kind separately. One uses Lemma B.2(e) in the former case and Lemma B.2(d) in the latter one, together with the assumption that $\Psi: C_{A}^{\geqslant 0}(J) \longrightarrow C^{\geqslant 0}(B)$ is an exact functor between exact categories.

Notice that the derived functor $\mathbb{R} \Psi: D^{\star}(A) \longrightarrow D^{\star}(B)$ that we have constructed does not depend on the choice of an integer $d$ satisfying the condition that $\Psi\left(C_{A}^{\geqslant 0}(J)\right)$ is contained in $\mathrm{C}^{\geqslant 0}(\mathrm{~B})^{\leqslant d}$. Indeed, for any bicomplex $J^{\bullet \bullet \bullet}$ with $J^{\bullet, i} \in \mathrm{C}_{\mathrm{A}}^{\geqslant 0}(\mathrm{~J})$ for all $i \in \mathbb{Z}$ and any integer $d^{\prime} \geqslant d$ a cone of the natural morphism between the totalizations of bicomplexes $\tau_{\leqslant d} \Psi\left(J^{\bullet \bullet \bullet}\right) \longrightarrow \tau_{\leqslant d^{\prime}} \Psi\left(J^{\bullet}, \bullet\right)$ is absolutely acyclic.

In the rest of this appendix, our aim is to establish an adjunction property of the left and right derived functors provided by the above construction. For any additive category $\mathrm{E}$, we denote by $\mathrm{C}^{-}(\mathrm{E})$ the DG-category of bounded above complexes in $\mathrm{E}$. When $E$ is an exact category, the subcategory $C^{\leqslant 0}(E) \subset C^{-}(E)$ of all the complexes $\cdots \longrightarrow E^{-2} \longrightarrow E^{-1} \longrightarrow E^{0} \longrightarrow 0$ and closed morphisms of degree zero between them is endowed with a natural exact category structure where a short sequence of complexes is exact if and only if it is termwise exact in $\mathrm{E}$.

Let $A$ and $B$ be two exact categories containing the images of idempotent endomorphisms of their objects. Let $\mathrm{J} \subset \mathrm{A}$ be a full subcategory, closed under extensions and the passages to the cokernels of admissible monomorphisms in $A$ and such that any object of $A$ is the source of an admissible monomorphism into an object of $J$. Similarly, let $\mathrm{P} \subset \mathrm{B}$ be a full subcategory, closed under extensions and the passages to the kernels of admissible epimorphisms and such that any object of $B$ is the target 
of an admissible epimorphism from an object of $\mathrm{P}$. The full subcategories $\mathrm{J}$ and $\mathrm{P}$ inherit the exact category structures of the ambient categories A and B.

Denote by $C_{P}^{\leqslant 0}(B)$ the full subcategory in $C \leqslant 0(P)$ consisting of all the complexes $\cdots \longrightarrow P^{-2} \longrightarrow P^{-1} \longrightarrow P^{0} \longrightarrow 0$ in $\mathrm{P}$ for which there exits an object $B \in \mathrm{B}$ together with a morphism $P^{0} \longrightarrow B$ such that the sequence $\cdots \longrightarrow P^{-1} \longrightarrow P^{0} \longrightarrow$ $B \longrightarrow 0$ is exact in $B$. The full subcategory $C_{\mathrm{B}}^{\leqslant 0}(P)$ is closed under extensions and the passages to the kernels of admissible epimorphisms in the exact category $C^{\leqslant 0}(P)$, so it inherits the exact category structure.

Let $\mathrm{C}^{\leqslant 0}(\mathrm{~A}) \geqslant-d \quad C \mathrm{C}^{\leqslant 0}(\mathrm{~A})$ denote the full subcategory consisting of all the complexes $\cdots \longrightarrow A^{-1} \longrightarrow A^{0} \longrightarrow 0$ in $\mathrm{A}$ such that the sequence $\cdots \longrightarrow A^{-d-2} \longrightarrow A^{-d-1} \longrightarrow$ $A^{-d}$ is exact in $\mathrm{A}$. Being closed under extensions and the passages to the kernels of admissible epimorphisms, the full subcategory $C^{\leqslant 0}(A) \geqslant-d$ inherits the exact category structure of the category $C^{\leqslant 0}(A)$. Denote by $C^{[-d, 0]}(A)$ the exact category of finite complexes $A^{-d} \longrightarrow \cdots \longrightarrow A^{0}$ in $\mathrm{A}$. Then there is an exact functor of canonical truncation $\tau_{\geqslant-d}: \mathrm{C}^{\leqslant 0}(\mathrm{~A}) \geqslant-d \longrightarrow \mathrm{C}^{[-d, 0]}(\mathrm{A})$.

As above, let $\Psi: \mathrm{C}^{+}(\mathrm{J}) \longrightarrow \mathrm{C}^{+}(\mathrm{B})$ be a DG-functor taking quasi-isomorphisms of complexes belonging to $C_{A}^{\geqslant 0}(J)$ to quasi-isomorphisms of complexes in the exact category B. Assume that the restriction of the functor $\Psi$ to the subcategory $\mathrm{C}_{A}^{\geqslant 0}(J) \subset$ $C^{+}(J)$ is an exact functor between exact categories $\Psi: C_{A}^{\geqslant 0}(J) \longrightarrow C^{\geqslant 0}(B) \leqslant d$. Then the above construction provides the right derived functors $\mathbb{R} \Psi: D^{\star}(A) \longrightarrow D^{\star}(B)$.

Similarly, let $\Phi: \mathrm{C}^{-}(\mathrm{P}) \longrightarrow \mathrm{C}^{-}(\mathrm{A})$ be a DG-functor taking quasi-isomorphisms of complexes belonging to $C_{B}^{\leqslant 0}(P)$ to quasi-isomorphisms of complexes in the exact category $A$. Assume that the restriction of the functor $\Phi$ to the subcategory $C_{B}^{\leqslant 0}(P) \subset$ $C^{-}(P)$ is an exact functor between exact categories $\Phi: C_{B}^{\leqslant 0}(P) \longrightarrow C^{\leqslant 0}(A) \geqslant-d$. Composing the restriction of the functor $\Phi$ with the functor of canonical truncation, we obtain an exact functor

$$
\tau_{\geqslant-d} \Phi: \mathrm{C}_{\mathrm{B}}^{\leqslant 0}(\mathrm{P}) \longrightarrow \mathrm{C}^{[-d, 0]}(\mathrm{A}) .
$$

The construction dual to the above provides a left derived functor

$$
\mathbb{L} \Phi: \mathrm{D}^{\star}(\mathrm{B}) \longrightarrow \mathrm{D}^{\star}(\mathrm{A})
$$

for any symbol $\star=b,+,-, \varnothing$, abs + , abs-, or abs. When the exact categories $A$ and $B$ have exact functors of infinite direct sum (resp., product), the full subcategory $\mathrm{P} \subset \mathrm{B}$ is closed under infinite direct sums (resp., products), and the functor $\Phi$ preserves infinite direct sums (resp., products), there is also the derived functor $\mathbb{L} \Phi$ acting between the coderived categories $\mathrm{D}^{\mathrm{co}}$ (resp., the contraderived categories $\mathrm{D}^{\mathrm{ctr}}$ ) of the exact categories $B$ and $A$.

Denote by $C(A)$ and $C(B)$ the DG-categories of unbounded complexes in the additive categories $A$ and $B$. Suppose that the DG-functors $\Psi: \mathrm{C}^{+}(\mathrm{J}) \longrightarrow \mathrm{C}^{+}(\mathrm{B})$ and $\Phi: C^{-}(P) \longrightarrow C^{-}(A)$, viewed as partially defined DG-functors between the DG-categories $C(A)$ and $C(B)$, are partially adjoint to each other. In other words, this means that for any complexes $J^{\bullet} \in \mathrm{C}^{+}(\mathrm{J})$ and $P^{\bullet} \in \mathrm{C}^{-}(\mathrm{P})$ there is an isomorphism

$$
\operatorname{Hom}_{\mathrm{C}(\mathrm{A})}^{\bullet}\left(\Phi\left(P^{\bullet}\right), J^{\bullet}\right) \simeq \operatorname{Hom}_{\mathrm{C}(\mathrm{B})}^{\bullet}\left(P^{\bullet}, \Psi\left(J^{\bullet}\right)\right)
$$


of complexes of morphisms in the DG-categories $\mathrm{C}(\mathrm{A})$ and $\mathrm{C}(\mathrm{B})$, functorial in the complexes $J^{\bullet}$ and $P^{\bullet}$. Let us show that in this case the derived functor $\mathbb{L} \Phi$ is left adjoint to the derived functor $\mathbb{R} \Psi$.

Our approach consists in constructing the adjunction morphisms

$$
\mathbb{L} \Phi \circ \mathbb{R} \Psi \longrightarrow \text { id and id } \longrightarrow \mathbb{R} \Psi \circ \mathbb{L} \Phi \text {. }
$$

Since the functors $\Phi$ and $\Psi$, being DG-functors, commute with totalizations of finite complexes of complexes in $\mathrm{C}^{-}(\mathrm{P})$ and $\mathrm{C}^{+}(\mathrm{J})$, the question essentially reduces to the case of single objects of the categories $A$ and $B$ viewed as one-term complexes along the grading $i$. So in the reasoning below we ignore the (generally speaking, doubly unbounded) grading $i$ altogether and concentrate on the (bounded on one side or finite) gradings created by the resolution procedures.

Let $0 \longrightarrow J^{0} \longrightarrow J^{1} \longrightarrow \cdots$ be a right resolution of an object $A \in$ A by objects $J^{j} \in \mathrm{J}$. Denote by $B^{1} \longrightarrow \cdots \longrightarrow B^{d}$ the finite complex $\tau_{\leqslant d} \Psi\left(J^{\bullet}\right)$ in the category B.

Let $\cdots \longrightarrow P^{-1, \bullet} \longrightarrow P^{0, \bullet} \longrightarrow B^{\bullet} \longrightarrow 0$ be an exact sequence of complexes in the category $\mathrm{B}$ with the terms $P^{k, j} \in \mathrm{P}$ concentrated in the interval of gradings $0 \leqslant j \leqslant d$. Then the total complex of the bicomplex $\tau_{\geqslant-d} \Phi\left(P^{\bullet \bullet \bullet}\right)$, where both the functor $\Phi$ and the canonical truncation are applied to complexes along the grading $k$, represents the object $\mathbb{L} \Phi(\mathbb{R} \Psi(A))$ in the derived category $\mathrm{D}^{\star}(\mathrm{A})$.

The composition $P^{\bullet \bullet} \longrightarrow B^{\bullet} \longrightarrow \Psi\left(J^{\bullet}\right)$ is a closed morphism from the total complex of the bicomplex $P^{\bullet \bullet \bullet}$ to the complex $\Psi\left(J^{\bullet}\right)$ in the category B. Consider the corresponding closed morphism of complexes $\Phi\left(P^{\bullet}, \bullet\right) \longrightarrow J^{\bullet}$ in the category A. Since the bicomplex $P^{k, j}$ is concentrated in the cohomological gradings $0 \leqslant j \leqslant d$ and $k \leqslant 0$, while the complex $J^{\bullet}$ is concentrated in the cohomological gradings $j \geqslant 0$, the latter morphism factorizes through the admissible epimorphism between the totalizations of bicomplexes $\tau_{\geqslant-d} \Phi\left(P^{\bullet \bullet \bullet}\right) \longrightarrow \Phi\left(P^{\bullet}, \bullet\right)$ and the admissible monomorphism of complexes $\tau_{\leqslant d} J^{\bullet} \longrightarrow J^{\bullet}$. We have obtained a closed morphism $\tau_{\geqslant-d} \Phi\left(P^{\bullet} \bullet \bullet\right) \tau_{\leqslant d} J^{\bullet}$ of complexes in the category A representing the desired adjunction morphism $\mathbb{L} \Phi(\mathbb{R} \Psi(A)) \longrightarrow A$.

The adjunction morphism id $\longrightarrow \mathbb{R} \Psi \circ \mathbb{L} \Phi$ of functors on the category $\mathrm{D}^{\star}(\mathrm{B})$ is constructed similarly. Checking that the compositions $\mathbb{R} \Psi \longrightarrow \mathbb{R} \Psi \circ \mathbb{L} \Phi \circ \mathbb{R} \Psi \longrightarrow \mathbb{R} \Psi$ and $\mathbb{L} \Phi \longrightarrow \mathbb{L} \Phi \circ \mathbb{R} \Psi \circ \mathbb{L} \Phi \longrightarrow \mathbb{L} \Phi$ are the identity morphisms is straightforward.

\section{REFERENCES}

[1] P. Balmer, M. Schlichting. Idempotent completion of triangulated categories. Journ. Algebra 236, \#2, p. 819-834, 2001.

[2] M. Boekstedt, A. Neeman. Homotopy limits in triangulated categories. Compositio Math. 86, \#2, p. 209-234, 1993.

[3] T. Bühler. Exact categories. Expositiones Math. 28, \#1, p. 1-69, 2010. arXiv:0811.1480 [math.HO]

[4] P. Deligne. Cohomologie à supports propres. SGA4, Tome 3. Lecture Notes in Math. 305, Springer-Verlag, Berlin-Heidelberg-New York, 1973, p. 250-480.

[5] M. Demazure. Lectures on p-divisible groups. Lecture Notes in Math. 302, Springer, 1972. 
[6] W. G. Dwyer, J. P. C. Greenlees. Complete modules and torsion modules. American Journ. of Math. 124, \#1, p. 199-220, 2002.

[7] A. I. Efimov, L. Positselski. Coherent analogues of matrix factorizations and relative singularity categories. Algebra and Number Theory 9, \#5, p. 1159-1292, 2015. arXiv:1102.0261 [math. CT]

[8] R. Hartshorne. Residues and duality. Lecture Notes in Math. 20, Springer, 1966.

[9] S. Iyengar, H. Krause. Acyclicity versus total acyclicity for complexes over noetherian rings. Documenta Math. 11, p. 207-240, 2006.

[10] P. Jørgensen. The homotopy category of complexes of projective modules. Advances in Math. 193, \#1, p. 223-232, 2005. arXiv:math.RA/0312088

[11] M. Kontsevich, A. Rosenberg. Noncommutative smooth spaces. The Gelfand Mathematical Seminars 1996-1999, p. 85-108, Birkhäuser Boston, Boston, MA, 2000. arXiv:math.AG/9812158

[12] H. Krause. The stable derived category of a Noetherian scheme. Compositio Math. 141, \#5, p. 1128-1162, 2005. arXiv:math.AG/0403526

[13] E. Matlis. Injective modules over Noetherian rings. Pacific Journ. of Math. 8, \#3, p. 511-528, 1958.

[14] E. Matlis. The higher properties of $R$-sequences. Journ. of Algebra 50, \#1, p. 77-112, 1978.

[15] D. Murfet. The mock homotopy category of projectives and Grothendieck duality. Ph. D. Thesis, Australian National University, September 2007. Available from http://www. therisingsea. org/thesis.pdf.

[16] A. Neeman. The Grothendieck duality theorem via Bousfield's techniques and Brown representability. Journ. of the American Math. Society 9, p. 205-236, 1996.

[17] A. Neeman. The homotopy category of flat modules, and Grothendieck duality. Inventiones Math. 174, p. 225-308, 2008.

[18] M. Porta, L. Shaul, A. Yekutieli. On the homology of completion and torsion. Algebras and Representation Theory 17, \#1, p. 31-67, 2014. arXiv:1010.4386 [math.AC] . Erratum in Algebras and Representation Theory 18, \#5, p. 1401-1405, 2015. arXiv:1506.07765 [math. AC]

[19] M. Porta, L. Shaul, A. Yekutieli. Completion by derived double centralizer. Algebras and Representation Theory 17, \#2, p. 481-494, 2014. arXiv:1207.0612 [math.AC]

[20] M. Porta, L. Shaul, A. Yekutieli. Cohomologically cofinite complexes. Communications in Algebra 43, \#2, p. 597-615, 2015. arXiv:1208.4064 [math.AC]

[21] L. Positselski. Homological algebra of semimodules and semicontramodules: Semi-infinite homological algebra of associative algebraic structures. Appendix $\mathrm{C}$ in collaboration with D. Rumynin; Appendix D in collaboration with S. Arkhipov. Monografie Matematyczne vol. 70, Birkhäuser/Springer Basel, 2010. xxiv+349 pp. arXiv:0708.3398 [math.CT]

[22] L. Positselski. Two kinds of derived categories, Koszul duality, and comodule-contramodule correspondence. Memoirs of the American Math. Society 212, \#996, 2011. vi+133 pp. arXiv:0905.2621 [math.CT]

[23] L. Positselski. Weakly curved $\mathrm{A}_{\infty}$-algebras over a topological local ring. Electronic preprint arXiv:1202.2697 [math.CT] .

[24] L. Positselski. Contraherent cosheaves. Electronic preprint arXiv:1209.2995 [math.CT] .

[25] L. Positselski. Comodule-contramodule correspondence. Slides of the presentation at the meeting in Třešt', Czech Republic, April 2014. Expanded version. Available from http://positselski.narod.ru/co-contra.pdf or http://positselski.narod.ru/cocontra-nopause.pdf.

[26] L. Positselski. Contramodules. Electronic preprint arXiv:1503.00991 [math.CT] .

[27] L. Positselski. Coherent rings, fp-injective modules, dualizing complexes, and covariant SerreGrothendieck duality. Electronic preprint arXiv:1504.00700 [math.CT] .

[28] L. Positselski. Dedualizing complexes of bicomodules and MGM duality over coalgebras. In preparation. 
[29] R. Rouquier. Dimensions of triangulated categories. Journ. of K-theory 1, \#2, p. 193-256, 2008. Erratum: same issue, p. 257-258. arXiv:math.CT/0310134

[30] P. Schenzel. Proregular sequences, local cohomology, and completion. Math. Scand. 92, \#2, p. 161-180, 2003.

[31] L. Shaul. Derived Hochschild functors over commutative adic algebras. Electronic preprint arXiv: 1307.5658 [math.AC].

[32] A. Yekutieli. Smooth formal embeddings and the residue complex. Canadian Journ. of Math. 50, \#4, p. 863-896, 1998. arXiv:alg-geom/9510007.

[33] A. Yekutieli. On flatness and completion for infinitely generated modules over noetherian rings. Communications in Algebra 39, \#11, p. 4221-4245, 2010. arXiv:0902.4378 [math.AC]

Department of Mathematics, Faculty of Natural Sciences, University of Haifa, Mount Carmel, Haifa 31905, IsRael; AND

Laboratory of Algebraic Geometry, National Research University Higher School of ECONOMICS, MOSCOW 117312; AND

Sector of Algebra and Number Theory, Institute for Information Transmission Problems, Moscow 127051, Russia

E-mail address: posic@mccme.ru 